\title{
Componentwise energy amplification in channel flows
}

\author{
By MIHAILO R. JOVANOVIĆ ${ }^{1}$ AND BASSAM BAMIEH \\ ${ }^{1}$ Department of Electrical and Computer Engineering, University of Minnesota, \\ Minneapolis, MN 55455, USA \\ ${ }^{2}$ Department of Mechanical Engineering, University of California, Santa Barbara, \\ CA 93106-5070, USA
}

(Received 21 February 2004 and in revised form 28 November 2004)

We study the linearized Navier-Stokes (LNS) equations in channel flows from an input-output point of view by analysing their spatio-temporal frequency responses. Spatially distributed and temporally varying body force fields are considered as inputs, and components of the resulting velocity fields are considered as outputs into these equations. We show how the roles of Tollmien-Schlichting (TS) waves, oblique waves, and streamwise vortices and streaks in subcritical transition can be explained as input-output resonances of the spatio-temporal frequency responses. On the one hand, we demonstrate the effectiveness of input field components, and on the other, the energy content of velocity perturbation components. We establish that wall-normal and spanwise forces have much stronger influence on the velocity field than streamwise force, and that the impact of these forces is most powerful on the streamwise velocity component. We show this using the relative scaling of the different input-output system components with the Reynolds number. We further demonstrate that for the streamwise constant perturbations, the spanwise force localized near the lower wall has, by far, the strongest effect on the evolution of the velocity field.

\section{Introduction}

In this paper, we analyse the dynamical properties of the Navier-Stokes (NS) equations with spatially distributed and temporally varying body force fields. These fields are considered as inputs, and different combinations of the resulting velocity fields are considered as outputs. This input-output analysis can in principle be done in any geometry and for the full nonlinear NS equations. In such generality, however, it is difficult to obtain useful results. We therefore concentrate on the geometry of channel flows, and the input-output dynamics of the linearized Navier-Stokes (LNS) equations.

The terminology here might be a little confusing. While we are concerned with the dynamical behaviour of the linearized NS equations, input-output analysis of the LNS is related, but not equivalent, to linear hydrodynamic stability analysis. In the latter, the objective is to characterize exponentially growing (that is, unstable) normal modes. As we will illustrate, input-output analysis reveals unstable normal modes, as well as transient growth, disturbance amplification, and so-called pseudospectral modes. This analysis has very close mathematical connections with the work on transient energy growth (also known as non-normal growth) (Gustavsson 1991; Butler \& Farrell 1992; Reddy \& Henningson 1993), pseudospectral analysis (Trefethen 
et al. 1993), and amplification of stochastic excitations (Farrell \& Ioannou 1993b; Bamieh \& Dahleh 2001). In some sense, it can also be considered as a type of receptivity analysis, although this connection is more qualitative. Rather than studying the receptivity of a boundary layer to upstream or free-stream disturbances as is commonly done (Goldstein \& Hultgren 1989; Hill 1995; Luchini \& Bottaro 1998), our input-output analysis is the receptivity of channel flows to spatially and temporally distributed body forces. The latter is mathematically and computationally more tractable, yet appears to have many of the qualitative features of boundary-layer receptivity analysis.

Our work is greatly influenced by recent work in what has become known as transient growth mechanisms for bypass transitions. We will only briefly outline some of the more closely related work here, and refer the reader to Schmid \& Henningson (2001) and the references therein for a fuller discussion. The main point of departure of this work from classical linear hydrodynamic stability is the fact that the latter is concerned solely with the existence of exponentially growing modes. In other words, it is essentially an asymptotic analysis of infinite time limits. In certain flows, however, transient (that is, finite-time) phenomena appear to play a significant role. While the possibility of transient growth has long been recognized (Orr 1907), it is only in the past two decades that effective mathematical methods have been used to analyse it. In Butler \& Farrell (1992) and Reddy \& Henningson (1993), initial states with the largest transient energy growth in subcritical flows were discovered using a singular value analysis. These 'worst case' initial states lead to flow structures that resemble streamwise vortices and streaks. A somewhat different analysis is done by computing the pseudospectrum rather than the spectrum of the generating dynamics (Trefethen et al. 1993), and the most unstable pseudospectral modes turn out to be related to streamwise vortices and streaks. A third analysis method (Farrell \& Ioannou 1993b; Bamieh \& Dahleh 2001) studies the most energetic response of the linearized NavierStokes equations to stochastic excitation. Yet again, the most energetically excited flow structures appear to resemble streamwise vortices and streaks. The common theme between the three methods is that a certain norm of the perturbed flow state is used (namely kinetic energy density), and the responses with respect to various types of uncertainties are analysed.

Previous work on 'non-normal growth' has tended to emphasize transient energy growth as a mechanism that might lead to exiting a region of attraction of the full nonlinear NS equations. In this paper, however, (and similarly in Farrell \& Ioannou 1993b; Bamieh \& Dahleh 2001), we adopt a different perspective in which the LNS are considered with uncertain body forces, initial conditions are set to zero, and thus flow-field perturbations are solely due to excitation by body forces. We show that the effects of these body forces on flow perturbations scale unfavourably with the Reynolds number. Depending on the force polarization and on how flow perturbations are measured, these effects scale with $R, R^{2}$ or $R^{3}$. Thus for large enough Reynolds numbers, the effects of even tiny body forces will become appreciable. It is not difficult to argue that any flow condition has some degree of uncertainty in either flow geometry, base profile and/or non-flat walls. In carefully controlled experiments, these uncertainties may be very small. However, it appears that the NS equations (both the linearized and full versions) in wall-bounded shear flows are exceedingly sensitive to small amounts of such uncertainty. It is thus important to analyse the equations while taking such uncertainties into account. We borrow heavily from the field of robust control theory (Zhou, Doyle \& Glover 1996), where mathematical tools for the analysis and control of uncertain dynamical systems have 


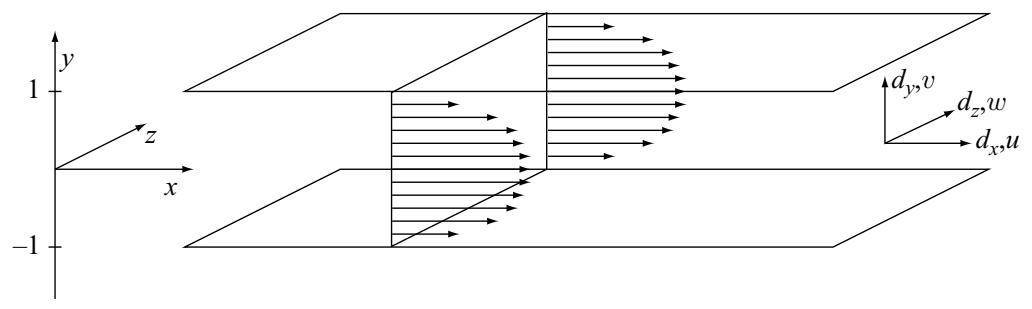

FIGURE 1. Three-dimensional flow between two parallel infinitely long plates.

been developed. Indeed, other researchers (Bewley \& Liu 1998; Lee et al. 2001; Kim 2003; Högberg et al. 2003a, b) have been successful in designing linear controllers based on these methods for either linearized or nonlinear flow dynamics in direct numerical simulations (DNS).

Our paper is organized as follows: in $\S 2$, we give a dynamical description of the flow fluctuations, introduce a notion of a spatio-temporal frequency response, and define different frequency response quantities that can be determined based on it. In $\S 3$, we present different portions of the spatio-temporal frequency response for subcritical Poiseuille flow at $R=2000$. One of our main objectives is to illustrate the inputoutput resonances from forcing inputs in different directions to different components of the velocity field. The most amplified input-output resonances turn out to resemble streamwise vortices and streaks and oblique waves. A comparison between them and internal resonances (Tollmien-Schlichting (TS) waves) is given using the frequency response. One of our other conclusions is that the spanwise and wall-normal forces are much more influential on flow perturbations than the streamwise force. Furthermore, the impact of these forces is most powerful on the streamwise velocity component. Analytical explanations for these observations as well as formulae for the dependence of these influences on the Reynolds number are given in $\S 4$. In $\S 5$, we study the effectiveness of the near-wall inputs for streamwise constant three-dimensional perturbations. We demonstrate analytically that the spanwise force has, by far, the biggest impact on the evolution of the velocity field components. These facts were recently observed in experimental and numerical studies of flow control using the Lorentz force (Henoch \& Stace 1995; Crawford \& Karniadakis 1997; Berger et al. 2000; Du \& Karniadakis 2000; Du, Symeonidis \& Karniadakis 2002). We conclude by remarking on the utility of frequency response analysis, and, more generally, analysis of effects of uncertainty on transitional and fully turbulent flows and their control.

\section{Dynamical description of flow fluctuations and input-output analysis}

We consider the incompressible LNS equations in channel-flow geometry shown in figure 1. These equations describe the dynamics (up to first order) of velocity and pressure fluctuations $\boldsymbol{u}$ and $p$, respectively. These are fluctuations about a nominal flow condition $(\overline{\boldsymbol{u}}, \bar{p})$. The equations can be written as

$$
\begin{aligned}
\partial_{t} \boldsymbol{u} & =-\nabla_{\bar{u}} \boldsymbol{u}-\nabla_{u} \overline{\boldsymbol{u}}-\nabla p+\frac{1}{R} \Delta \boldsymbol{u}+\boldsymbol{d}, \\
0 & =\nabla \cdot \boldsymbol{u},
\end{aligned}
$$

where $\boldsymbol{u}:=\left[\begin{array}{lll}u & v & w\end{array}\right]^{T}$ is the fluctuation velocity vector, $p$ is pressure fluctuation, $\boldsymbol{d}$ is a spatially distributed and temporally varying body force, $R$ is the Reynolds number, $\nabla$ 
is the gradient, $\Delta:=\nabla^{2}$ is the Laplacian, and the operator $\nabla_{u}$ is given by $\nabla_{u}:=\boldsymbol{u} \cdot \nabla$. Each field is assumed to vary both temporally and spatially, e.g. $\boldsymbol{d}=\boldsymbol{d}(x, y, z, t)$. The additional ingredient in these equations is the body force field $\boldsymbol{d}$ which we consider to be an 'external' input or excitation in these equations.

We rewrite these equations in a form that is more amenable to the input-output analysis. This is done by a standard conversion (Kim, Moin \& Moser 1987) into an evolution equation for the wall-normal velocity $(v)$ and vorticity $\left(\omega_{y}\right)$ fields. At any point in time, the remaining velocity and vorticity fields can be expressed in terms of these two fields using spatial differential operators. This conversion is done as follows. An equation for $p$ can be obtained by applying the divergence operator to $(2.1 a)$ and combining the resulting equation with $(2.1 b)$. In this way, $p$ can be eliminated from (2.1), and we can obtain a PDE for the evolution of $v$. A PDE for the evolution of $\omega_{y}$ is obtained by taking the curl of (2.1a). This yields two PDEs for the evolution of wall-normal velocity $v$ and vorticity $\omega_{y}$ that involve only $v, \omega_{y}$ and $\boldsymbol{d}$. If nominal flow $\overline{\boldsymbol{u}}$ is constant in the $x$ and $z$ directions, then the underlying operators have coefficients that are constant in these directions. In this situation, the analysis is greatly simplified by applying a Fourier transform in horizontal (streamwise and spanwise) directions. The overall equations can then be re-written as

$$
\begin{aligned}
\partial_{t} \boldsymbol{\psi}\left(k_{x}, y, k_{z}, t\right) & =\left[\mathscr{A}\left(k_{x}, k_{z}\right) \boldsymbol{\psi}\left(k_{x}, k_{z}, t\right)\right](y)+\left[\mathscr{B}\left(k_{x}, k_{z}\right) \boldsymbol{d}\left(k_{x}, k_{z}, t\right)\right](y), \\
\boldsymbol{\phi}\left(k_{x}, y, k_{z}, t\right) & =\left[\mathscr{C}\left(k_{x}, k_{z}\right) \boldsymbol{\psi}\left(k_{x}, k_{z}, t\right)\right](y),
\end{aligned}
$$

where $\boldsymbol{\psi}:=\left[\begin{array}{ll}\psi_{1} & \psi_{2}\end{array}\right]^{T}=\left[\begin{array}{ll}v & \omega_{y}\end{array}\right]^{T}, \boldsymbol{d}:=\left[\begin{array}{lll}d_{x} & d_{y} & d_{z}\end{array}\right]^{T}, \boldsymbol{\phi}:=\boldsymbol{u}=\left[\begin{array}{lll}u & v & w\end{array}\right]^{T}$, and $\mathscr{A}, \mathscr{B}$ and $\mathscr{C}$ are operators defined below. Note that the forces in the streamwise, wall-normal and spanwise directions are represented by $d_{x}, d_{y}$ and $d_{z}$, respectively, and that we use the same symbol to denote a field and its Fourier transform. The reason for writing the equations in the above form is to regard the vector field $\psi$ as the 'state' of the system (from which any other field can be determined at a given point in time), $\boldsymbol{d}$ as an input, and $\boldsymbol{\phi}$ as an output. This is the so-called state-space form of driven dynamical systems common in the dynamics and controls literature (Curtain \& Zwart 1995). We have chosen here the velocity field vector as the output. It is also possible to chose any combination of velocity and vorticity fields as an output, resulting in different choices for operator $\mathscr{C}$.

For a nominal flow condition $(\overline{\boldsymbol{u}}, \bar{p}):=\left(\left[\begin{array}{lll}U(y) & 0 & 0\end{array}\right]^{T}, \bar{p}(x)\right)$, operators $\mathscr{A}, \mathscr{B}$ and $\mathscr{C}$ are given by

$$
\begin{aligned}
\mathscr{A} & :=\left[\begin{array}{ll}
\mathscr{A}_{11} & 0 \\
\mathscr{A}_{21} & \mathscr{A}_{22}
\end{array}\right]:=\left[\begin{array}{cc}
\left(-\mathrm{i} k_{x} \Delta^{-1} U \Delta+\mathrm{i} k_{x} \Delta^{-1} U^{\prime \prime}+(1 / R) \Delta^{-1} \Delta^{2}\right) \\
\left(-\mathrm{i} k_{z} U^{\prime}\right)
\end{array}\right. \\
\mathscr{B} & :=\left[\begin{array}{lll}
\mathscr{B}_{x} & \mathscr{B}_{y} & \mathscr{B}_{z}
\end{array}\right]:=\left[\begin{array}{cc}
\Delta^{-1} & 0 \\
0 & I
\end{array}\right]\left[\begin{array}{ccc}
-\mathrm{i} k_{x} \partial_{y} & -\left(k_{x}^{2}+k_{z}^{2}\right) & -\mathrm{i} k_{z} \partial_{y} \\
\mathrm{i} k_{z} & 0 & -\mathrm{i} k_{x}
\end{array}\right], \\
\mathscr{C} & :=\left[\begin{array}{l}
\mathscr{C}_{u} \\
\mathscr{C}_{v} \\
\mathscr{C}_{w}
\end{array}\right]:=\frac{1}{k_{x}^{2}+k_{z}^{2}}\left[\begin{array}{cc}
\mathrm{i} k_{x} \partial_{y} & -\mathrm{i} k_{z} \\
k_{x}^{2}+k_{z}^{2} & 0 \\
\mathrm{i} k_{z} \partial_{y} & \mathrm{i} k_{x}
\end{array}\right],
\end{aligned}
$$

where $U^{\prime}:=\mathrm{d} U(y) / \mathrm{d} y$, and $\Delta:=\partial_{y y}-k_{x}^{2}-k_{z}^{2}$. Note that each of the operators $\mathscr{A}$, $\mathscr{B}$ and $\mathscr{C}$ is one-dimensional (in the wall-normal direction). All three operators (and 
consequently system (2.2)) are parameterized by three parameters: the streamwise and spanwise wavenumbers $k_{x}$ and $k_{z}$, and the Reynolds number $R$. Finally, the boundary conditions on $v$ and $\omega_{y}$ are derived from the original no-slip boundary conditions and they are given by

$$
v\left(k_{x}, \pm 1, k_{z}, t\right)=\partial_{y} v\left(k_{x}, \pm 1, k_{z}, t\right)=\omega_{y}\left(k_{x}, \pm 1, k_{z}, t\right)=0, \quad \forall k_{x}, k_{z} \in \mathbb{R}, \quad \forall t \geqslant 0 .
$$

We note here that the LNS equations with input (2.2) that we use are qualitatively similar to those used by Farrell \& Ioannou (1993b), but with an important difference. In Farrell \& Ioannou (1993b), forcing was introduced directly to the right-hand side of the $\left(v, \omega_{y}\right)$ equations. In our set-up, the forcing is introduced in the more basic LNS equations, which results in a more transparent interpretation of the excitation as body forces, and allows for a detailed analysis of the effects of polarized forces. The mathematical difference is that the forcing in Farrell \& Ioannou (1993b) has only two components and enters the equations through a different operator $\mathscr{B}$.

In the sequel, we will also analyse the situation in which external excitation $\boldsymbol{d}$ has an intensity that varies with the wall-normal direction. This is used to model the situation where the forcing fields have higher intensity near walls, as might be the case in certain flow-control problems. A more general form for the forcing fields is

$$
\boldsymbol{d}(x, y, z, t):=\left[\begin{array}{ccc}
\kappa_{x}(y) & 0 & 0 \\
0 & \kappa_{y}(y) & 0 \\
0 & 0 & \kappa_{z}(y)
\end{array}\right] \boldsymbol{d}_{0}(x, y, z, t)=\left[\begin{array}{c}
\kappa_{x}(y) d_{x 0}(x, y, z, t) \\
\kappa_{y}(y) d_{y 0}(x, y, z, t) \\
\kappa_{z}(y) d_{z 0}(x, y, z, t)
\end{array}\right]
$$

where $\kappa_{s}(y)(s=x, y$, or $z)$ are certain pre-specified functions of $y$ representing the relative intensities of the forcing fields in the wall-normal direction, and $\boldsymbol{d}_{0}(x, y, z, t)$ is an arbitrary function of $x, y, z$ and $t$ with a spatial Fourier transform $\boldsymbol{d}_{0}\left(k_{x}, y, k_{z}, t\right)$. Note that $\boldsymbol{d}_{0}$ is considered 'uniformly' distributed in the wall-normal direction in a sense to be described in the sequel. It is convenient to incorporate the functions $\kappa_{s}$ in operator $\mathscr{B}$ by defining

$$
\mathscr{B}_{0}:=\mathscr{B}\left[\begin{array}{ccc}
\kappa_{x} & 0 & 0 \\
0 & \kappa_{y} & 0 \\
0 & 0 & \kappa_{z}
\end{array}\right]=\left[\begin{array}{llll}
\mathscr{B}_{x} \kappa_{x} & \mathscr{B}_{y} \kappa_{y} & \mathscr{B}_{z} \kappa_{z}
\end{array}\right]=:\left[\begin{array}{lll}
\mathscr{B}_{x 0} & \mathscr{B}_{y 0} & \mathscr{B}_{z 0}
\end{array}\right] .
$$

Then, (2.2) can be rewritten as

$$
\begin{aligned}
\partial_{t} \boldsymbol{\psi}\left(k_{x}, y, k_{z}, t\right) & =\left[\mathscr{A}\left(k_{x}, k_{z}\right) \boldsymbol{\psi}\left(k_{x}, k_{z}, t\right)\right](y)+\left[\mathscr{B}_{0}\left(k_{x}, k_{z}\right) \boldsymbol{d}_{0}\left(k_{x}, k_{z}, t\right)\right](y), \\
\boldsymbol{\phi}\left(k_{x}, y, k_{z}, t\right) & =\left[\mathscr{C}\left(k_{x}, k_{z}\right) \boldsymbol{\psi}\left(k_{x}, k_{z}, t\right)\right](y) .
\end{aligned}
$$

Note that the equations are again in the state-space form, but with a different operator acting on the input. We respectively refer to systems (2.2) and (2.6) as the systems with 'unstructured' and 'structured' external excitations. This reflects the fact that in the latter, operator $\mathscr{B}$ has the wall-normal intensity profile of the forcing field built into it.

Certain mathematical considerations are required for a precise description of the above operators. They are included in Appendix A.

\subsection{Spatio-temporal frequency responses}

We will study the spatio-temporal frequency response of the LNS with input (2.2). We introduce this notion as follows. Let the input to (2.2) be a field that is harmonic 
in the $t, x$, and $z$ variables, that is of the form

$$
\boldsymbol{d}(x, y, z, t)=\overline{\boldsymbol{d}}(y) \mathrm{e}^{\mathrm{i}\left(\bar{k}_{x} x+\bar{k}_{z} z+\bar{\omega} t\right)},
$$

where $\overline{\boldsymbol{d}}(y)$ is some function of $y$. Assuming stability of generator $\mathscr{A}$, it can then be shown that the output is also harmonic in the same variables and is determined by

$$
\boldsymbol{\phi}(x, y, z, t)=\left[\mathscr{H}\left(\bar{k}_{x}, \bar{k}_{z}, \bar{\omega}\right) \overline{\boldsymbol{d}}\right](y) \mathrm{e}^{\mathrm{i}\left(\bar{k}_{x} x+\bar{k}_{z} z+\bar{\omega} t\right)},
$$

where operator $\mathscr{H}\left(k_{x}, k_{z}, \omega\right)$ is given by

$$
\mathscr{H}\left(k_{x}, k_{z}, \omega\right)=\mathscr{C}\left(k_{x}, k_{z}\right)\left(\mathrm{i} \omega \boldsymbol{I}-\mathscr{A}\left(k_{x}, k_{z}\right)\right)^{-1} \mathscr{B}\left(k_{x}, k_{z}\right) .
$$

There are two possible interpretations of this. If this harmonic input is assumed to act over the time interval $-\infty<t<\infty$, then initial states do not play a role, and the output is precisely given by (2.7). If, on the other hand, the harmonic input is assumed to act over $0 \leqslant t<\infty$ with zero initial state $(\psi(t=0)=0)$, then the output limits to (2.7) as transients asymptotically die out (owing to stability) in the 'steady-state' (that is, as $t \rightarrow \infty$ ).

Note that for each $\left(k_{x}, k_{z}, \omega\right), \mathscr{H}\left(k_{x}, k_{z}, \omega\right)$ is a one-dimensional operator in the wall-normal direction. The operator-valued function $\mathscr{H}\left(k_{x}, k_{z}, \omega\right)$ in $(2.8)$ is called the spatio-temporal frequency response of system (2.2). It is a function of temporal frequency $\omega$ and two spatial wavenumbers $k_{x}$ and $k_{z}$. Another interpretation of this function is that it maps the Fourier transform of the input to the Fourier transform of the output, i.e.

$$
\boldsymbol{\phi}\left(k_{x}, y, k_{z}, \omega\right)=\left[\mathscr{H}\left(k_{x}, k_{z}, \omega\right) \boldsymbol{d}\left(k_{x}, \cdot, k_{y}, \omega\right)\right](y) .
$$

The frequency response (2.8) contains a large amount of information about the dynamical behaviour of system (2.2). It is not always straightforward to visualize $\mathscr{H}$ since it is a function of three frequency variables, and is also operator valued (or matrix valued after a suitable discretization in the wall-normal direction). In this paper, we are mainly interested in the behaviour of the response as a function of the two spatial wave numbers. Therefore, we must somehow aggregate the effects of temporal and wall-normal dynamics. This can be done in a variety of ways. We choose to use the so-called $\mathscr{H}_{2}$ and $\mathscr{H}_{\infty}$ system norms (Zhou et al. 1996). These are input-output norms of dynamical systems of the type (2.2), and they quantify the 'amplification' or 'gain' of a system, or in other words, the relative size of the output to the input.

The $\mathscr{H}_{2}$ norm is defined by

$$
\left[\|\mathscr{H}\|_{2}^{2}\right]\left(k_{x}, k_{z}\right):=\frac{1}{2 \pi} \int_{-\infty}^{\infty}\left\|\mathscr{H}\left(k_{x}, k_{z}, \omega\right)\right\|_{H S}^{2} \mathrm{~d} \omega,
$$

where $\|\cdot\|_{H S}$ is the Hilbert-Schmidt norm of an operator defined by

$$
\|\mathscr{H}\|_{H S}^{2}:=\operatorname{trace}\left(\mathscr{H} \mathscr{H}^{*}\right) \text {. }
$$

In the case when $\mathscr{H}$ is a matrix, $\|\cdot\|_{H S}$ is precisely the Frobenius norm.

The $\mathscr{H}_{\infty}$ norm is defined by

$$
\left[\|\mathscr{H}\|_{\infty}\right]\left(k_{x}, k_{z}\right):=\sup _{\omega} \sigma_{\max }\left(\mathscr{H}\left(k_{x}, k_{z}, \omega\right)\right),
$$

where $\sigma_{\max }$ is the maximum singular value.

These norms have appealing physical interpretations. We can regard $\mathscr{H}$ in (2.9) as representing the 'amplification' or 'gain' from $\boldsymbol{d}$ to $\boldsymbol{\phi}$ at given frequencies. The 
quantity (2.11) represents a maximization of this gain over input temporal frequencies and wall-normal shapes (by this we mean the variation of the input as a function of $y$ ). The former is obtained by the maximization over $\omega$ and the latter by taking a maximum singular value. Thus, we can interpret the $\mathscr{H}_{\infty}$ norm as the worst case amplification of deterministic inputs. Furthermore, we can find a frequency $\bar{\omega}$ (the frequency at which $\sup _{\omega}$ is achieved) and input shape $\overline{\boldsymbol{d}}(y)$ (namely, the right singular function corresponding to the maximum singular value) such that with input

$$
\boldsymbol{d}(x, y, z, t)=\overline{\boldsymbol{d}}(y) \mathrm{e}^{\mathrm{i}\left(k_{x} x+k_{z} z+\bar{\omega} t\right)},
$$

the output $\boldsymbol{\phi}$ has this worst case amplification, that is

$$
\int_{0}^{\infty} \int_{-1}^{1} \boldsymbol{\phi}^{*}\left(k_{x}, y, k_{z}, t\right) \boldsymbol{\phi}\left(k_{x}, y, k_{z}, t\right) \mathrm{d} y \mathrm{~d} t=\left[\|\mathscr{H}\|_{\infty}\right]\left(k_{x}, k_{z}\right) .
$$

On the other hand, the $\mathscr{H}_{2}$ norm has an interesting stochastic interpretation: it quantifies the variance (energy) amplification of harmonic (in $x$ and $z$ ) stochastic (in $y$ and $t$ ) disturbances at any given $\left(k_{x}, k_{z}\right)$. In the fluid mechanics literature, the $\mathscr{H}_{2}$ norm is also referred to as the ensemble average energy density of the statistical steady state (Farrell \& Ioannou 1993b). More generally, the frequency response operator captures the mapping of second-order statistics from the input to the output random fields. Let input $\boldsymbol{d}$ be a zero-mean homogenous (in $x, z$ and $t$ ) white random field, i.e. one whose auto-correlation is

$$
\mathscr{E}\left\{\boldsymbol{d}\left(x_{1}, y_{1}, z_{1}, t_{1}\right) \boldsymbol{d}^{*}\left(x_{2}, y_{2}, z_{2}, t_{2}\right)\right\}=\boldsymbol{I} \delta\left(x_{1}-x_{2}, y_{1}-y_{2}, z_{1}-z_{2}, t_{1}-t_{2}\right),
$$

where $\boldsymbol{I}$ is the $3 \times 3$ identity matrix, and $\delta$ is the Dirac delta function. The output random field will be stationary in $x, z$ and $t$ (but not in $y$ ), and will have correlations determined by the system's dynamics. We define the auto-correlation function of $\boldsymbol{\phi}$ after averaging in the wall-normal direction by

$$
R_{\phi}(x, z, t):=\int_{-1}^{1} \int_{-1}^{1} \mathscr{E}\left\{\boldsymbol{\phi}\left(\xi+x, y_{1}, \zeta+z, \tau+t\right) \boldsymbol{\phi}^{*}\left(\xi, y_{2}, \zeta, \tau\right)\right\} \mathrm{d} y_{1} \mathrm{~d} y_{2}
$$

Note that $R_{\phi}$ contains all the two-point correlation functions in $x, z$ and $t$. Let $W_{\phi}$ be the power spectral density (PSD) function of $\boldsymbol{\phi}$ obtained by a Fourier transform of $R_{\phi}$. It is then a standard fact (VanMarcke 1983) that the PSD is determined from the system's frequency response by

$$
W_{\phi}\left(k_{x}, k_{z}, \omega\right):=\operatorname{trace}\left(\mathscr{H}\left(k_{x}, k_{z}, \omega\right) \mathscr{H}^{*}\left(k_{x}, k_{z}, \omega\right)\right)=\left\|\mathscr{H}\left(k_{x}, k_{z}, \omega\right)\right\|_{H S}^{2} .
$$

Comparing this last expression with (2.10), we see that the $\mathscr{H}_{2}$ norm at each $\left(k_{x}, k_{z}\right)$ captures the total PSD at these wavenumbers after integrating in temporal frequency. For a more detailed analysis of the statistics of the LNS, we refer the reader to Jovanović \& Bamieh (2001).

We point out that for computing the $\mathscr{H}_{2}$ norm, (2.10) can be determined without actually integrating in $\omega$. It is a standard fact from linear systems theory (Zhou et al. 1996) that this quantity can be determined using the solutions of the operator Lyapunov equations

$$
\begin{aligned}
& \mathscr{A}\left(k_{x}, k_{z}\right) \mathscr{X}\left(k_{x}, k_{z}\right)+\mathscr{X}\left(k_{x}, k_{z}\right) \mathscr{A}^{*}\left(k_{x}, k_{z}\right)=-\mathscr{B}\left(k_{x}, k_{z}\right) \mathscr{B}^{*}\left(k_{x}, k_{z}\right), \\
& \mathscr{A}^{*}\left(k_{x}, k_{z}\right) \mathscr{Y}\left(k_{x}, k_{z}\right)+\mathscr{Y}\left(k_{x}, k_{z}\right) \mathscr{A}\left(k_{x}, k_{z}\right)=-\mathscr{C}^{*}\left(k_{x}, k_{z}\right) \mathscr{C}\left(k_{x}, k_{z}\right),
\end{aligned}
$$

where $\mathscr{A}^{*}, \mathscr{B}^{*}$ and $\mathscr{C}^{*}$ represent adjoints of operators $\mathscr{A}, \mathscr{B}$ and $\mathscr{C}$ (these operators are precisely defined in Appendix A). The $\mathscr{H}_{2}$ norm is then determined from either 
of the following two expressions

$\left[\|\mathscr{H}\|_{2}^{2}\right]\left(k_{x}, k_{z}\right)=\operatorname{trace}\left(\mathscr{X}\left(k_{x}, k_{z}\right) \mathscr{C}^{*}\left(k_{x}, k_{z}\right) \mathscr{C}\left(k_{x}, k_{z}\right)\right)=\operatorname{trace}\left(\mathscr{Y}\left(k_{x}, k_{z}\right) \mathscr{B}\left(k_{x}, k_{z}\right) \mathscr{B}^{*}\left(k_{x}, k_{z}\right)\right)$.

In the controls literature, operators $\mathscr{X}\left(k_{x}, k_{z}\right)$ and $\mathscr{Y}\left(k_{x}, k_{z}\right)$ are, respectively, referred to as the controllability and observability Gramians (Zhou et al. 1996).

Finally, one of our main aims in this paper is to perform a comparative analysis of the effects of forcing in the three different coordinate directions on each of the three velocity fields. We thus need a more detailed analysis of the frequency response from each input to each output. Note that the input and output operators in (2.3) have three subcomponents corresponding to each of the inputs and outputs, respectively. This induces a $3 \times 3$ matrix decomposition of the frequency response operator as follows

$$
\begin{aligned}
& \mathscr{H}\left(k_{x}, k_{z}, \omega\right)=\left[\begin{array}{c}
\mathscr{C}_{u} \\
\mathscr{C}_{v} \\
\mathscr{C}_{w}
\end{array}\right]\left(\mathrm{i} \omega \boldsymbol{I}-\mathscr{A}\left(k_{x}, k_{z}\right)\right)^{-1}\left[\mathscr{B}_{x} \quad \mathscr{B}_{y} \quad \mathscr{B}_{z}\right] \\
& =:\left[\begin{array}{ccc}
\mathscr{H}_{u x}\left(k_{x}, k_{z}, \omega\right) & \mathscr{H}_{u y}\left(k_{x}, k_{z}, \omega\right) & \mathscr{H}_{u z}\left(k_{x}, k_{z}, \omega\right) \\
\mathscr{H}_{v x}\left(k_{x}, k_{z}, \omega\right) & \mathscr{H}_{v y}\left(k_{x}, k_{z}, \omega\right) & \mathscr{H}_{v z}\left(k_{x}, k_{z}, \omega\right) \\
\mathscr{H}_{w x}\left(k_{x}, k_{z}, \omega\right) & \mathscr{H}_{w y}\left(k_{x}, k_{z}, \omega\right) & \mathscr{H}_{w z}\left(k_{x}, k_{z}, \omega\right)
\end{array}\right],
\end{aligned}
$$

where, for example, $\mathscr{H}_{v z}$ is the response from the forcing $d_{z}$ to the $v$ velocity field, and similarly for the other 8 responses. By combining the rows and columns of this $3 \times 3$ matrix, we obtain another decomposition as follows

$$
\begin{aligned}
& \mathscr{H}\left(k_{x}, k_{z}, \omega\right)=\mathscr{C}(\mathrm{i} \omega \boldsymbol{l}-\mathscr{A})^{-1}\left[\mathscr{B}_{x} \quad \mathscr{B}_{y} \quad \mathscr{B}_{z}\right] \\
& =:\left[\mathscr{H}_{x}\left(k_{x}, k_{z}, \omega\right) \quad \mathscr{H}_{y}\left(k_{x}, k_{z}, \omega\right) \quad \mathscr{H}_{z}\left(k_{x}, k_{z}, \omega\right)\right] \\
& =\left[\begin{array}{c}
\mathscr{C}_{u} \\
\mathscr{C}_{v} \\
\mathscr{C}_{w}
\end{array}\right](\mathrm{i} \omega \boldsymbol{I}-\mathscr{A})^{-1} \mathscr{B}=:\left[\begin{array}{c}
\mathscr{H}_{u}\left(k_{x}, k_{z}, \omega\right) \\
\mathscr{H}_{v}\left(k_{x}, k_{z}, \omega\right) \\
\mathscr{H}_{w}\left(k_{x}, k_{z}, \omega\right)
\end{array}\right] \text {, }
\end{aligned}
$$

where for example $\mathscr{H}_{v}$ is the response from all three inputs to the $v$ field, while $\mathscr{H}_{x}$ is the response from $d_{x}$ to all three velocity fields.

In this paper, we are mainly concerned with $\mathscr{H}_{2}$-normlike quantities for systems (2.2) and (2.6). The numerical results for the $\mathscr{H}_{\infty}$ norms are qualitatively similar to those for the $\mathscr{H}_{2}$ norm (Jovanovic 2004). However, the latter is more amenable to theoretical analysis in that we are able to obtain analytical expressions for the variances at $k_{x}=0$, and we therefore concentrate on the $\mathscr{H}_{2}$ norm case.

\section{Frequency responses in subcritical Poiseuille flow with $R=2000$}

In this section, we consider the NS equations linearized around a nominal velocity profile of the form $U(y)=1-y^{2}$ at $R=2000$. We calculate the $\mathscr{H}_{2}$ norms from different body force inputs to different velocity outputs as functions of spatial wavenumbers, and discuss the corresponding three-dimensional flow structures that are most amplified. These turn out to be typically streamwise elongated/spanwise periodic and oblique. We show how the roles of TS waves, oblique waves and streamwise vortices and streaks can be explained as input-output resonances of the spatio-temporal frequency responses. We further analyse the effectiveness of input field components, and the energy content of $u, v$ and $w$. Also, we demonstrate that 

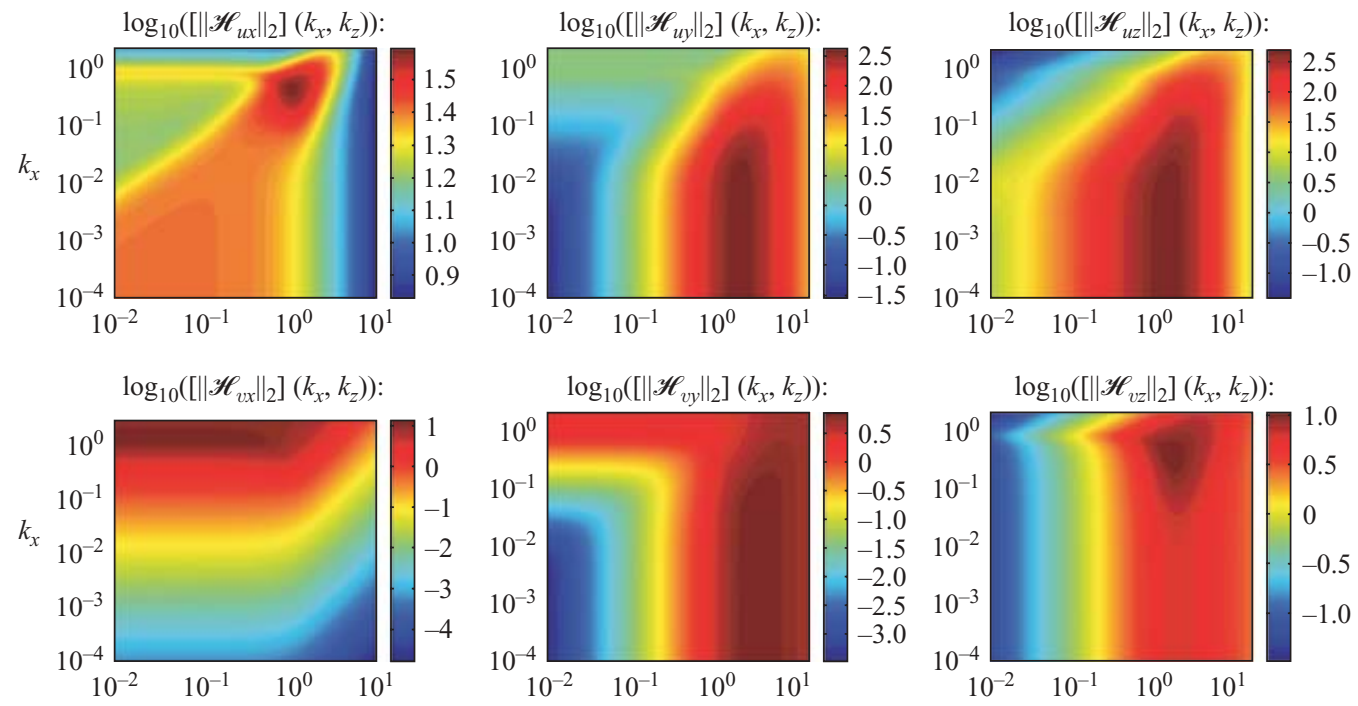

$\log _{10}\left(\left[\left\|\mathscr{H}_{w x}\right\|_{2}\right]\left(k_{x}, k_{z}\right)\right)$ :
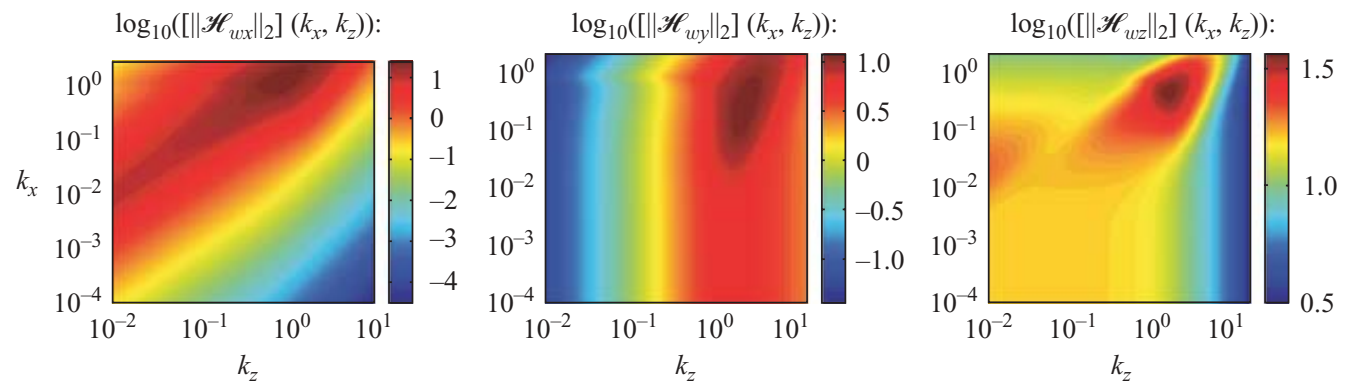

FIgURE 2. Plots of $\left[\left\|\mathscr{H}_{r s}\right\|_{2}\right]\left(k_{x}, k_{z}\right)$ in Poiseuille flow with $R=2000$.

the wall-normal and spanwise forces have much stronger influence on the velocity field than the streamwise force, and that the impact of these forces is most powerful on the streamwise velocity component.

All results presented here are obtained numerically using the scheme described in Appendix $\mathrm{C}$, with $30 v$ and $\omega_{y}$ basis functions $(N=M=29)$. By increasing the number of basis functions, it is confirmed that this resolution is high enough $\dagger$. The $\mathscr{H}_{2}$-normlike quantities are determined based on solutions of the corresponding Lyapunov equations, with $50 \times 90$ grid points in the wavenumber space $\left(k_{x}, k_{z}\right)$. These points are chosen in the logarithmic scale with $\left(k_{x \min }:=10^{-4}, k_{x \max }:=3.02\right)$ and $\left(k_{\text {zmin }}:=10^{-2}, k_{\text {zmax }}:=15.84\right)$.

The $\left(k_{x}, k_{z}\right)$-parameterized $\mathscr{H}_{2}$ norms of all components of the frequency response operator are illustrated in figure 2. Different plots correspond to the different elements of the operator valued matrix on the right-hand side of (2.12). We make the following observations.

$\dagger$ We observe that in contrast to eigenvalue computations for generator $\mathscr{A}$ (which are used in stability calculations), much less resolution is required for computing system norms. While typically about 100 points might be required in a collocation scheme for stability calculations in channel flows, about 20-30 points are adequate to compute norms with very good accuracy. This is perhaps a reflection of the fact that norms are more 'robust' quantities than eigenvalues in channel-flow problems. 
Input-output amplifications from $d_{y}$ and $d_{z}$ to $u$ attain the largest values. Clearly, both $\left[\left\|\mathscr{H}_{u y}\right\|_{2}\right]\left(k_{x}, k_{z}\right)$ and $\left[\left\|\mathscr{H}_{u z}\right\|_{2}\right]\left(k_{x}, k_{z}\right)$ have fairly similar shapes with distinctive peaks whose values are at least one order of magnitude larger than the peak values of all other quantities shown in figure 2. This illustrates that the wall-normal and spanwise forces have much stronger influence on the velocity field than the streamwise force, and that the impact of these forces is most powerful on the streamwise velocity component.

Different components of frequency response peak at different locations in the $\left(k_{x}, k_{z}\right)$ plane. Hence, the componentwise input-output analysis uncovers several distinct amplification mechanisms for subcritical transition. These mechanisms are responsible for creation of streamwise vortices and streaks (peak values at $k_{x} \approx 0, k_{z} \approx O(1)$ ), oblique waves (peak values at $k_{x} \approx O(1), k_{z} \approx O(1)$ ), and TS waves (peak values at $\left.k_{x} \approx O(1), k_{z} \approx 0\right)$.

The streamwise elongated structures are most amplified by the system's dynamics, followed by the oblique perturbations, followed by the TS waves. Clearly, there is about an order of magnitude difference between the largest peak values taking place at $\left(k_{x} \approx 0, k_{z} \approx O(1)\right)$ and at $\left(k_{x} \approx O(1), k_{z} \approx O(1)\right)$. On the other hand, in Poiseuille flow with $R=2000$ the oblique waves are approximately four times more amplified than the least-stable LNS modes (TS waves). However, this is not to say that the TS waves and especially the oblique waves should be neglected in studies of transition. Either of them can trigger nonlinear terms in the full NS equations. Moreover, the phase of these perturbations can play an important role when the flow is connected in feedback with, for example, a controller for drag reduction.

We note that the importance of the streamwise vortices and streaks was thoroughly studied by Gustavsson (1991); Butler \& Farrell (1992); Farrell \& Ioannou (1993b); Reddy \& Henningson (1993); Trefethen et al. (1993); Bamieh \& Dahleh (2001), among others. The fact that the oblique waves often produce the greatest transient amplification was recognized by Farrell \& Ioannou (1993a) in the study of the three-dimensional perturbations to viscous constant shear flow that give the largest energy growth on a selected time interval. Also, a Karhunen-Loève analysis of a direct numerical simulation of turbulent channel flow at $R_{\tau}=80$ revealed the presence of energetic oblique waves (Sirovich, Ball \& Keefe 1990). Oblique wave transition scenario was introduced by Schmid \& Henningson (1992). This transition scenario was studied experimentally by Elofsson \& Alfredsson (1998) in Poiseuille flow, and both experimentally and numerically by Berlin, Wiegel \& Henningson (1999) in boundary layers. The transition thresholds in oblique plane channel flow transition were examined by Reddy et al. (1998), and recently by Chapman (2002). The identification of the oblique waves as the input-output resonances illustrates usefulness of the componentwise input-output approach to the analysis of the problem at hand.

Figure 3 shows the $\mathscr{H}_{2}$ norms of different components of frequency response operator (2.13). These plots quantify the amplification from inputs in the three spatial directions to the velocity vector $\phi=\left[\begin{array}{lll}u & v & w\end{array}\right]^{T}$. The square additive property of the $\mathscr{H}_{2}$ norm implies that these plots can be obtained by summing the rows in figure 2. We note that:

The external excitations applied in the wall-normal and spanwise directions have a much bigger impact on the velocity field than the streamwise direction forcing. The explanation for this observation is given in $\S 4$, where we show that the energy of three-dimensional streamwise constant perturbations in the presence of either $d_{y}$ or $d_{z}$ achieves $O\left(R^{3}\right)$ amplification. On the other hand, only amplification proportional 

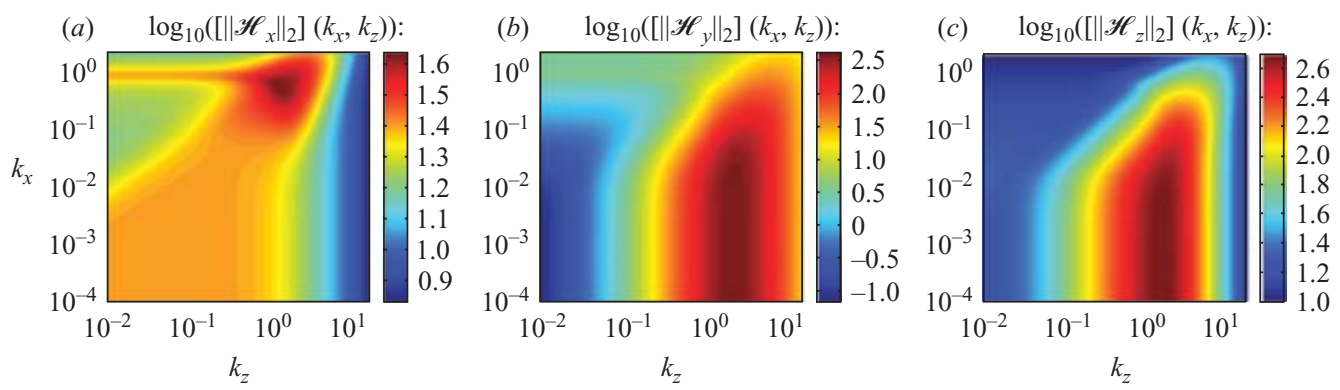

FIgURE 3. Plots of $\left[\left\|\mathscr{H}_{x}\right\|_{2}\right]\left(k_{x}, k_{z}\right),\left[\left\|\mathscr{H}_{y}\right\|_{2}\right]\left(k_{x}, k_{z}\right)$, and $\left[\left\|\mathscr{H}_{z}\right\|_{2}\right]\left(k_{x}, k_{z}\right)$, in Poiseuille flow with $R=2000$.
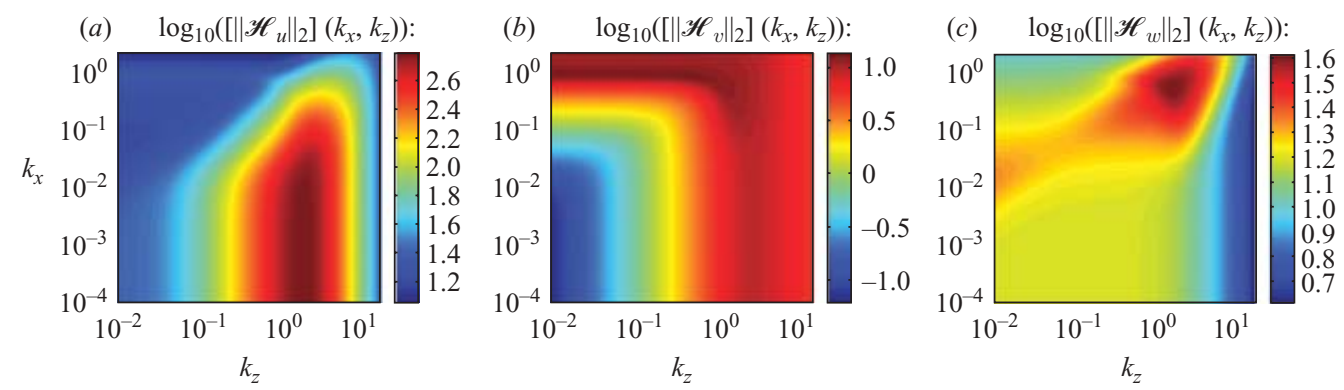

FIGURE 4. Plots of $\left[\left\|\mathscr{H}_{u}\right\|_{2}\right]\left(k_{x}, k_{z}\right),\left[\left\|\mathscr{H}_{v}\right\|_{2}\right]\left(k_{x}, k_{z}\right)$, and $\left[\left\|\mathscr{H}_{w}\right\|_{2}\right]\left(k_{x}, k_{z}\right)$, in Poiseuille flow with $R=2000$.

to the Reynolds number is achievable when external force in the streamwise direction is applied, as illustrated in $\S 4$.

Functions $\left[\left\|\mathscr{H}_{y}\right\|_{2}\right]\left(k_{x}, k_{z}\right)$ and $\left[\left\|\mathscr{H}_{z}\right\|_{2}\right]\left(k_{x}, k_{z}\right)$ achieve their largest values at the different locations in the $\left(k_{x}, k_{z}\right)$-plane than function $\left[\left\|\mathscr{H}_{x}\right\|_{2}\right]\left(k_{x}, k_{z}\right)$. Clearly, the former two quantities peak at $k_{x}=0$ for certain $O(1)$ value of $k_{z}$. These input-output resonances correspond to the streamwise vortices and streaks. On the other hand, the latter quantity attains the global maximum at the location where both spatial wavenumbers have $O(1)$ values. We also observe a local peak at the streamwise wavenumber axis in figure $3(a)$. This peak is caused by the least-stable modes of $\mathscr{A}$ (TS waves). Even though the presence of the least-stable modes in figure $3(a)$ is more prominent than in figures $3(b)$ and $3(c)$, the structures that are more amplified by the system's dynamics are still three-dimensional. These structures correspond to the oblique waves.

Figure 4 illustrates the energy content of different velocity components by showing the $k_{x}-k_{z}$ dependence of the $\mathscr{H}_{2}$ norm of frequency response operator (2.14) These plots quantify the respective amplification from input vector $\boldsymbol{d}:=\left[\begin{array}{lll}d_{x} & d_{y} & d_{z}\end{array}\right]^{T}$ to velocity perturbations $u, v$ and $w$. The square additive property of the $\mathscr{H}_{2}$ norm implies that these plots can be obtained by summing the columns in figure 2 . We remark that:

The energy content of the streamwise velocity is much bigger than the energy content of the other two velocity components. The analytical explanation for this observation is given in $\S 4$, where we explicitly show that the variance of three-dimensional streamwise velocity perturbations at $k_{x}=0$ scales as $R^{3}$. This is in sharp contrast with the amplification that $v$ and $w$ experience. Namely, our derivations of $\S 4$ prove that the variance of streamwise constant wall-normal and spanwise velocity perturbations is only proportional to the Reynolds number. 


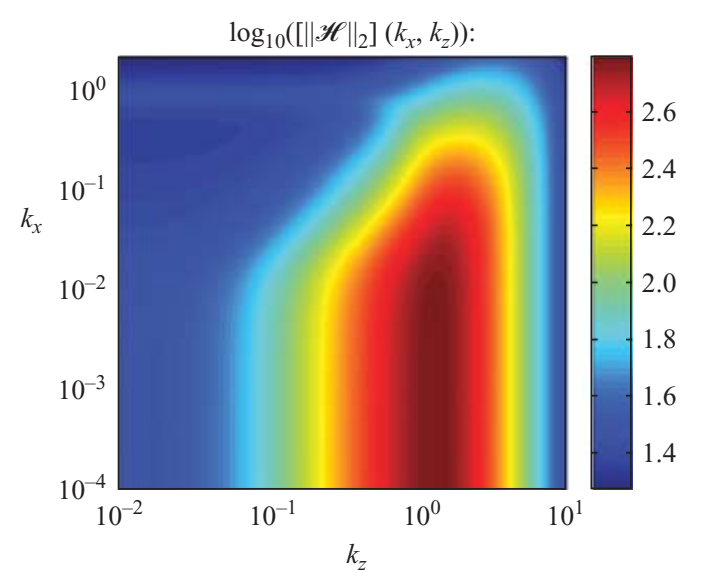

Figure 5. Plot of $\left[\|\mathscr{H}\|_{2}\right]\left(k_{x}, k_{z}\right)$ in Poiseuille flow with $R=2000$.

Functions $\left[\left\|\mathscr{H}_{u}\right\|_{2}\right]\left(k_{x}, k_{z}\right),\left[\left\|\mathscr{H}_{v}\right\|_{2}\right]\left(k_{x}, k_{z}\right)$ and $\left[\left\|\mathscr{H}_{w}\right\|_{2}\right]\left(k_{x}, k_{z}\right)$, respectively, achieve their largest values on the $k_{z}$-axis, $k_{x}$-axis, and at $\left(k_{x} \approx O(1), k_{z} \approx O(1)\right)$. These peaks correspond to the streamwise vortices and streaks (figure $4(a)$ ), the least-stable system modes (figure $4(b)$ ), and the oblique waves (figure $4(c)$ ). Since the evolution of the wallnormal velocity is governed by the stable Orr-Sommerfeld equation, it is not surprising that $\left[\left\|\mathscr{H}_{v}\right\|_{2}\right]\left(k_{x}, k_{z}\right)$ achieves largest values in the immediate vicinity of the least-stable modes. The local peak at $\left(k_{x}=0, k_{z} \approx O(1)\right)$ in figure $4(b)$ signals a potential for the transient amplification in the Orr-Sommerfeld equation. However, this amplification is significantly weaker than the amplification of streamwise elongated structures in figure $4(a)$ and oblique perturbations in figure $4(c)$. We further note that, since both $u$ and $w$ depend on $\omega_{y}$ (cf. $(2.3 c)$ ) they experience transient amplification due to a coupling between wall-normal velocity and vorticity perturbations (that is, a vortex stretching (vortex tilting) mechanism, Landahl 1975, 1980). Namely, the nominal spanwise vorticity (that is, $-U^{\prime}$ ) is tilted in the wall-normal direction by the spanwise changes in $v$ which leads to a transient amplification of $\omega_{y}$. Since for streamwise constant perturbations, $w$ depends only on $v$ (cf. $(2.3 c)$ ), the amplification of $w$ at $k_{x}=0$ is limited to $O(R)$, as shown in $\S 4$. Clearly, this amplification becomes larger when non-zero streamwise wavenumbers are considered. This can be attributed to the fact that away from the $k_{z}$-axis, $w$ is a function of both $v$ and $\omega_{y}(\mathrm{cf} .(2.3 c))$. The latter quantity achieves much bigger magnitudes than the former owing to the aforementioned vortex tilting mechanism which is responsible for the input-output resonances observed in figure $4(c)$. We note that this mechanism does not take place whenever the nominal shear is zero (that is, $U^{\prime}=0$ ), or when there is no spanwise variation in the velocity perturbations (that is, $k_{z}=0$ ).

In figure 5 , we show the $\mathscr{H}_{2}$ norm of frequency response operator (2.8). This plot quantifies the aggregate effect of forces in all three spatial directions to all three velocity components. Clearly, it is difficult to see different amplification mechanisms here, because of the dominance of the streamwise elongated structures. We remark that the TS waves generate a local peak at $\left(k_{x} \approx 1, k_{z}=0\right)$, with a magnitude significantly lower than that achieved by these prevailing streamwise elongated flow structures. On the other hand, the previously discussed peaks created by the oblique perturbations are inconspicuous in figure 5. This demonstrates the utility of componentwise analysis over the analysis that only accounts for the aggregate effects. 
The numerical computations of this section are strengthened by a rigorous analysis of the various $\mathscr{H}_{2}$ norms of the streamwise constant three-dimensional channel-flow perturbations. These analytical considerations are presented in $\S 4$.

\section{Dependence of variance amplification on the Reynolds number}

We now study the forced LNS equations for streamwise constant three-dimensional perturbations. In this special case, the model for velocity perturbations is usually referred to as the two-dimensional three-component (2D/3C) model (Reynolds \& Kassinos 1995). The motivation for analysis of this particular model is twofold. First, from numerical computations presented in $\S 3$, we observe that the streamwise constant perturbations experience utmost amplification rates. Secondly, this model is amenable to a thorough analysis which clarifies the relative strengths of amplification mechanisms from various forcing directions to velocity field components, as well as their dependence on the Reynolds number.

The governing dynamics of streamwise constant three-dimensional perturbations are obtained by setting $k_{x}=0$ in (2.2) which yields

$$
\begin{aligned}
\partial_{t}\left[\begin{array}{l}
\psi_{1} \\
\psi_{2}
\end{array}\right] & =\left[\begin{array}{cc}
\frac{1}{R} \mathscr{L} & 0 \\
\mathscr{C}_{p} & \frac{1}{R} \mathscr{S}
\end{array}\right]\left[\begin{array}{l}
\psi_{1} \\
\psi_{2}
\end{array}\right]+\left[\begin{array}{ccc}
0 & \mathscr{B}_{y 1} & \mathscr{B}_{z 1} \\
\mathscr{B}_{x 2} & 0 & 0
\end{array}\right]\left[\begin{array}{l}
d_{x} \\
d_{y} \\
d_{z}
\end{array}\right], \\
{\left[\begin{array}{c}
u \\
v \\
w
\end{array}\right] } & =\left[\begin{array}{cc}
0 & \mathscr{C}_{u 2} \\
\mathscr{C}_{v 1} & 0 \\
\mathscr{C}_{w 1} & 0
\end{array}\right]\left[\begin{array}{l}
\psi_{1} \\
\psi_{2}
\end{array}\right],
\end{aligned}
$$

with

$$
\begin{aligned}
\mathscr{L} & :=\Delta^{-1} \Delta^{2}, & \mathscr{S} & :=\Delta, & \mathscr{C}_{p} & :=-\mathrm{i} k_{z} U^{\prime}(y), \\
\mathscr{B}_{x 2} & :=\mathrm{i} k_{z}, & \mathscr{B}_{y 1} & :=-k_{z}^{2} \Delta^{-1}, & \mathscr{B}_{z 1} & :=-\mathrm{i} k_{z} \Delta^{-1} \partial_{y}, \\
\mathscr{C}_{u 2} & :=-\frac{\mathrm{i}}{k_{z}}, & \mathscr{C}_{v 1} & :=\boldsymbol{I}, & \mathscr{C}_{w 1} & :=\frac{\mathrm{i}}{k_{z}} \partial_{y} .
\end{aligned}
$$

These equations are parameterized by two parameters: the spanwise wavenumber $k_{z}$, and the Reynolds number $R$. The wall-normal velocity and vorticity fields are in this section respectively, denoted by $\psi_{1}\left(y, k_{z}, t\right)$ and $\psi_{2}\left(y, k_{z}, t\right)$, with the boundary conditions $\psi_{1}\left( \pm 1, k_{z}, t\right)=\partial_{y} \psi_{1}\left( \pm 1, k_{z}, t\right)=\psi_{2}\left( \pm 1, k_{z}, t\right)=0$. We note that the lower-block-triangular generator in (4.1a) is exponentially stable for any finite $R$ and any parallel flow $U(y)$. This follows from Appendix B, where the spectral analysis of the Reynolds-number/nominal-velocity independent, normalized Squire and OrrSommerfeld operators $\mathscr{S}$ and $\mathscr{L}$ is performed. Thus, the expressions for input-output gains that we derive in this section are valid for all Reynolds numbers and all nominal velocity profiles $U(y)$.

We first study the frequency responses (as a function of $k_{z}$ ) of each of the components of the transfer function (2.12). We do this using the $\mathscr{H}_{2}$ norm, that is

$$
\left[\left\|\mathscr{H}_{r s}\right\|_{2}^{2}\right]\left(k_{z}\right):=\frac{1}{2 \pi} \int_{-\infty}^{\infty}\left\|\mathscr{H}_{r s}\left(k_{z}, \omega\right)\right\|_{H S}^{2} \mathrm{~d} \omega \quad \text { for } r=u, v, w \text { and } s=x, y, z .
$$

We then investigate the dependence of each of the subsystems on the Reynolds number. We analytically demonstrate that amplification from both spanwise and 


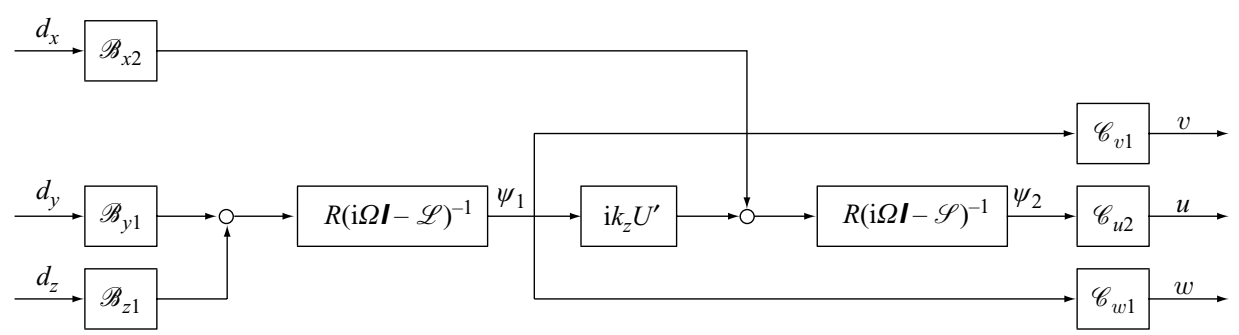

FIGURE 6. Block diagram of the streamwise constant LNS system (4.1) with $\Omega:=\omega R$.

wall-normal forcing to streamwise velocity is $O\left(R^{3}\right)$, while amplification for all other components is $O(R)$.

Application of the temporal Fourier transform allows us to represent system (4.1) in terms of its block diagram shown in figure 6 with $\Omega:=\omega R$. We note that the same temporal scaling was previously employed by Gustavsson (1991) in his undertaking to determine the transient growth of the wall-normal vorticity. Thus, from this block diagram, it follows that for the streamwise constant perturbations, the frequency responses from $d_{y}$ and $d_{z}$ to $u$ scale as $R^{2}$, whereas the responses from all other inputs to other velocity outputs scale at most linearly with $R$. In particular, at $k_{x}=0$, the streamwise forcing does not influence the wall-normal and the spanwise velocity components. It is noteworthy that the coupling term $\mathscr{C}_{p}=-\mathrm{i} k_{z} U^{\prime}$ is crucial for providing this $R^{2}$-scaling. Namely, we can observe from figure 6 that in the absence of shear or spanwise variations in the wall-normal velocity perturbation, all components of operator $\mathscr{H}\left(0, k_{z}, \omega\right)$ in $(2.12)$ are at most proportional to $R$. This further exemplifies the importance of the vortex stretching mechanism (Landahl 1975) in the wall-bounded shear flows. We remark that the numerical experiments of Kim \& Lim (2000) indicated that without the coupling from $v$ to $\omega_{y}$ the near-wall turbulence decays in a fully turbulent channel flow.

We now state the main result whose proof can be found in Appendix D. Theorem 1 quantifies the energy amplification for each of the components of frequency response (2.12) at $k_{x}=0$.

THEOREM 1. For any streamwise constant channel flow with nominal velocity $U(y)$, the $\mathscr{H}_{2}$ norms of operators $\mathscr{H}_{r s}\left(k_{z}, \omega, R\right)$ that map $d_{s}$ into $r,\{r=u, v, w ; s=x, y, z\}$, are given by

$$
\left[\begin{array}{lll}
{\left[\left\|\mathscr{H}_{u x}\right\|_{2}^{2}\right]\left(k_{z}\right)} & {\left[\left\|\mathscr{H}_{u y}\right\|_{2}^{2}\right]\left(k_{z}\right)} & {\left[\left\|\mathscr{H}_{u z}\right\|_{2}^{2}\right]\left(k_{z}\right)} \\
{\left[\left\|\mathscr{H}_{v x}\right\|_{2}^{2}\right]\left(k_{z}\right)} & {\left[\left\|\mathscr{H}_{v y}\right\|_{2}^{2}\right]\left(k_{z}\right)} & {\left[\left\|\mathscr{H}_{v z}\right\|_{2}^{2}\right]\left(k_{z}\right)} \\
{\left[\left\|\mathscr{H}_{w x}\right\|_{2}^{2}\right]\left(k_{z}\right)} & {\left[\left\|\mathscr{H}_{w y}\right\|_{2}^{2}\right]\left(k_{z}\right)} & {\left[\left\|\mathscr{H}_{w z}\right\|_{2}^{2}\right]\left(k_{z}\right)}
\end{array}\right]=\left[\begin{array}{ccc}
f_{u x}\left(k_{z}\right) R & g_{u y}\left(k_{z}\right) R^{3} & g_{u z}\left(k_{z}\right) R^{3} \\
0 & f_{v y}\left(k_{z}\right) R & f_{v z}\left(k_{z}\right) R \\
0 & f_{w y}\left(k_{z}\right) R & f_{w z}\left(k_{z}\right) R
\end{array}\right],
$$

where the $f$ and $g$ functions are independent of $R$.

The energy amplification of streamwise constant perturbations scales as $R^{3}$ from the forces in the wall-normal and the spanwise directions to the streamwise velocity. In all other cases, it scales at most as $R$. In particular, at $k_{x}=0$ the streamwise forcing does not influence the wall-normal and the spanwise velocity components. This further illustrates the dominance of the streamwise velocity perturbations and the forces in the remaining two spatial directions for high-Reynolds-number channel flows (figure 7). 

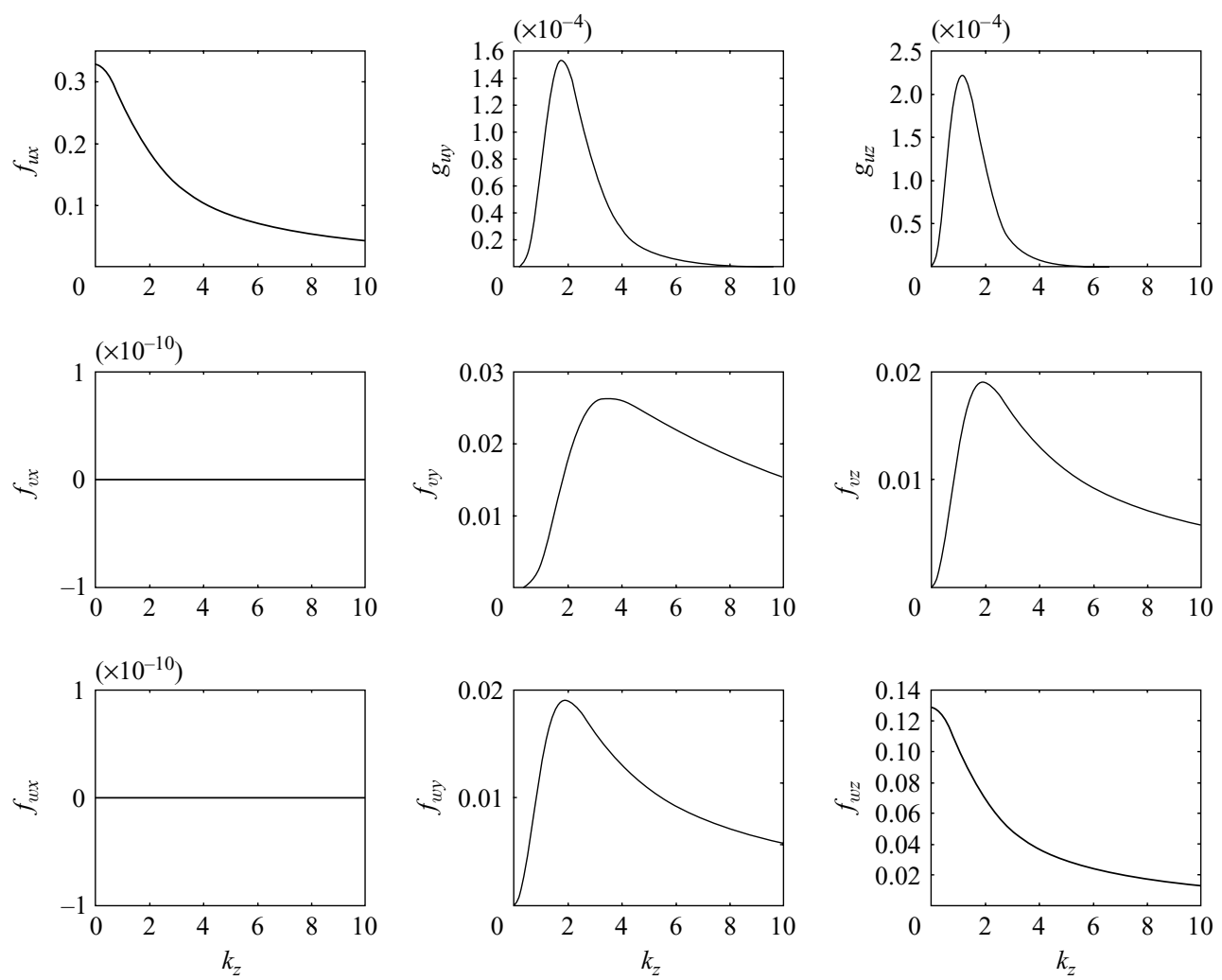

FIGURE 7. Plots of $f_{r s}\left(k_{z}\right),\{r=u, v, w ; s=x, y, z\}, g_{u y}\left(k_{z}\right)$ and $g_{u z}\left(k_{z}\right)$. Functions $g_{u y}\left(k_{z}\right)$ and $g_{u z}\left(k_{z}\right)$ are determined for Couette flow.

The expressions for the terms that multiply $R$ in (4.2) are the same for all channel flows, because these terms depend only on nominal-velocity independent operators $\mathscr{L}$ and $\mathscr{S}$ (see Appendix E for details). On the other hand, the expressions for $g_{u y}$ and $g_{u z}$ depend on the coupling operator (vortex stretching term from the NS equations) $\mathscr{C}_{p}:=-\mathrm{i} k_{z} U^{\prime}(y)$, and therefore these terms are nominal-velocity dependent. Whenever there is either no mean shear or no spanwise variations in the velocity perturbations, the vortex tilting mechanism is absent and the largest amplification that can be achieved is proportional to the Reynolds number.

The following corollary of Theorem 1 is obtained by summing the rows in (4.2) and exploiting the square additive property of the $\mathscr{H}_{2}$ norm. It quantifies the energy amplification of streamwise constant perturbations for each of the components of frequency response (2.13).

COROLlARY 2. For any streamwise constant channel flow with nominal velocity $U(y)$, the $\mathscr{H}_{2}$ norms of operators $\mathscr{H}_{s}\left(k_{z}, \omega, R\right)$ that map $d_{s}$ into $\phi:=\left[\begin{array}{lll}u & v & w\end{array}\right]^{T},\{s=x, y, z\}$, are given by

$$
\left.\begin{array}{l}
{\left[\left\|\mathscr{H}_{x}\right\|_{2}^{2}\right]\left(k_{z}\right)=f_{x}\left(k_{z}\right) R,} \\
{\left[\left\|\mathscr{H}_{y}\right\|_{2}^{2}\right]\left(k_{z}\right)=f_{y}\left(k_{z}\right) R+g_{y}\left(k_{z}\right) R^{3},} \\
{\left[\left\|\mathscr{H}_{z}\right\|_{2}^{2}\right]\left(k_{z}\right)=f_{z}\left(k_{z}\right) R+g_{z}\left(k_{z}\right) R^{3},}
\end{array}\right\}
$$

where $\left\{f_{x}:=f_{u x}, f_{y}:=f_{v y}+f_{w y}, f_{z}:=f_{v z}+f_{w z}\right\}$ and $\left\{g_{y}:=g_{u y}, g_{z}:=g_{u z}\right\}$. 

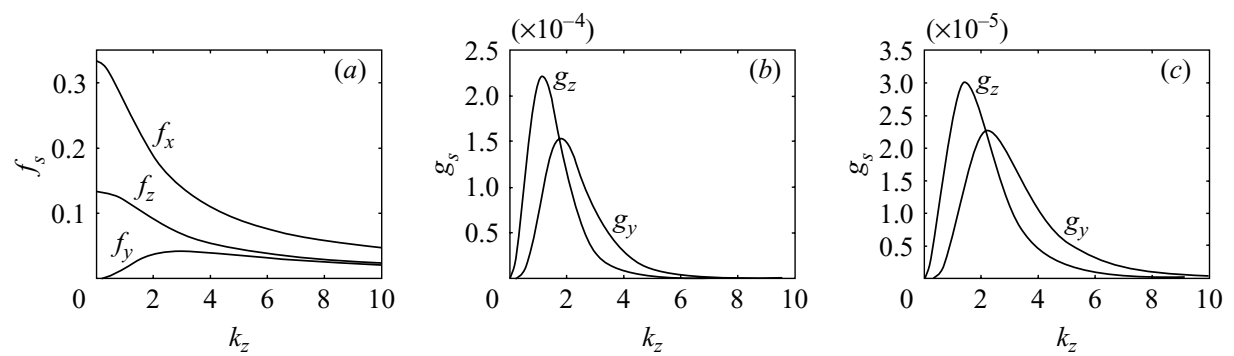

FIGURE 8 . The $k_{z}$-dependence of $f_{x}, f_{y}, f_{z}, g_{y}$ and $g_{z}$. Expressions for $f_{x}, f_{y}$ and $f_{z}$ are the same for all channel flows, as demonstrated in Appendix E.2.1. The terms responsible for the $O\left(R^{3}\right)$ energy amplification are shown in $(b)$ Couette flow and $(c)$ Poiseuille flow.

Figure 8 illustrates the $k_{z}$-dependence of functions $f_{x}, f_{y}, f_{z}, g_{y}$ and $g_{z}$. Expressions for $f_{x}, f_{y}$ and $f_{z}$ are the same for all channel flows, as shown in Appendix E.2.1 where we derive the analytical formulae for these quantities. On the other hand, both $g_{y}$ and $g_{z}$ depend on the nominal velocity. In Appendix E.2.4, we determine the analytical expressions for these two quantities in Couette flow by using the spectral decompositions of the Orr-Sommerfeld and Squire operators. These expressions are given in terms of rapidly convergent series and they are shown in figure $8(b)$. The numerically computed dependence of $g_{y}$ and $g_{z}$ on $k_{z}$ in Poiseuille flow is given in figure $8(c)$.

Therefore, as already indicated by the numerical computations of $\S 3$, the forces in the spanwise and wall-normal directions have the strongest influence on the velocity field. We confirmed this observation by analytical derivations summarized in Corollary 2 for the streamwise constant perturbations showing that the square of the $\mathscr{H}_{2}$ norm from $d_{z}$ and $d_{y}$ to velocity vector $\phi$ scales as $R^{3}$. On the other hand, at $k_{x}=0$, the square of the $\mathscr{H}_{2}$ norm from $d_{x}$ to $\phi$ scales as $R$.

The following corollary of Theorem 1 is obtained by summing the columns in (4.2) and exploiting the square additive property of the $\mathscr{H}_{2}$ norm. It quantifies the energy amplification of streamwise constant perturbations for each of the components of frequency response (2.14).

Corollary 3. For any streamwise constant channel flow with nominal velocity $U(y)$, the $\mathscr{H}_{2}$ norms of operators $\mathscr{H}_{r}\left(k_{z}, \omega, R\right)$ that map $\boldsymbol{d}:=\left[d_{x} d_{y} d_{z}\right]^{T}$ into $r,\{r=u, v, w\}$, are given by

$$
\left.\begin{array}{l}
{\left[\left\|\mathscr{H}_{u}\right\|_{2}^{2}\right]\left(k_{z}\right)=f_{u}\left(k_{z}\right) R+g_{u}\left(k_{z}\right) R^{3},} \\
{\left[\left\|\mathscr{H}_{v}\right\|_{2}^{2}\right]\left(k_{z}\right)=f_{v}\left(k_{z}\right) R,} \\
{\left[\left\|\mathscr{H}_{w}\right\|_{2}^{2}\right]\left(k_{z}\right)=f_{w}\left(k_{z}\right) R,}
\end{array}\right\}
$$

where $\left\{f_{u}:=f_{u x}, f_{v}:=f_{v y}+f_{v z}, f_{w}:=f_{w y}+f_{w z}\right\}$ and $g_{u}:=g_{u y}+g_{u z}$.

Figure 9 shows the $k_{z}$-dependence of functions $f_{u}, f_{v}, f_{w}$ and $g_{u}$. Expressions for $f_{u}, f_{v}$ and $f_{w}$ are the same for all channel flows, as demonstrated in Appendix E.2.2 where we derive the analytical formulae for these quantities. On the other hand, $g_{u}$ depends on the underlying mean velocity. In Appendix E.2.2, we derive the analytical dependence of $g_{u}$ on $k_{z}$ for Couette flow. Figure $9(c)$ illustrates the numerically computed $k_{z}$-dependence of $g_{u}$ in Poiseuille flow.

Thus, the impact of the external excitations is most powerful on the streamwise velocity component. We confirmed this observation by both numerical computations of $\S 3$ and analytical derivations summarized in Corollary 3 for the streamwise constant 

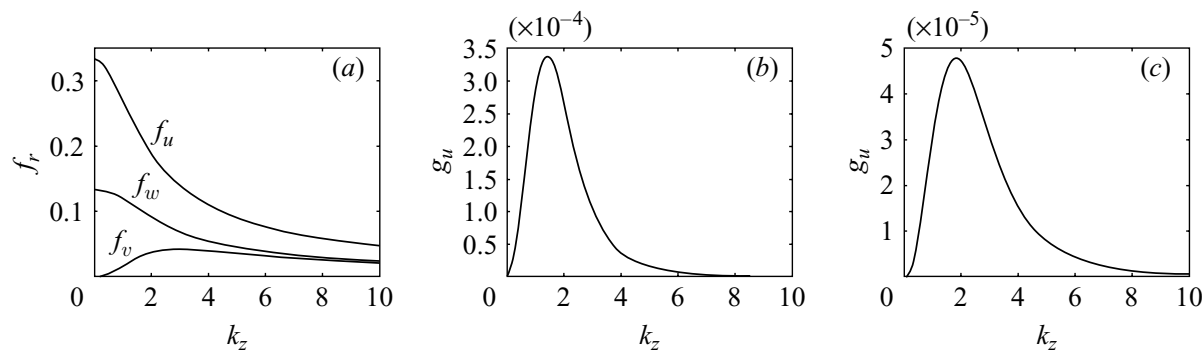

FigurE 9. The $k_{z}$-dependence of $f_{u}, f_{v}, f_{w}$ and $g_{u}$. Expressions for $f_{u}, f_{v}$ and $f_{w}$ are the same for all channel flows, as demonstrated in Appendix E.2.2. The terms responsible for the $O\left(R^{3}\right)$ energy amplification are shown in $(a)$ Couette flow and $(b)$ Poiseuille flow.

perturbations showing that the square of the $\mathscr{H}_{2}$ norm from $\boldsymbol{d}$ to $u$ scales as $R^{3}$. On the other hand, at $k_{x}=0$, the square of the $\mathscr{H}_{2}$ norm from $\boldsymbol{d}$ to $v$ and $w$ scales as $R$.

It is worth mentioning that Theorem 1 of Bamieh \& Dahleh (2001) follows from Theorem 1 of this section. Namely, by summing all elements in (4.2) and using the square-additivity of the $\mathscr{H}_{2}$ norm, the aggregate effect of all forces to all velocity components is readily obtained. For completeness, we state the main result of Bamieh \& Dahleh (2001) as the following corollary of Theorem 1.

COROLlary 4. For any streamwise constant channel flow with nominal velocity $U(y)$, the $\mathscr{H}_{2}$ norm of operator $\mathscr{H}\left(k_{z}, \omega, R\right)$ that maps $\boldsymbol{d}:=\left[\begin{array}{lll}d_{x} & d_{y} & d_{z}\end{array}\right]^{T}$ into $\boldsymbol{\phi}:=\left[\begin{array}{lll}u & v & w\end{array}\right]^{T}$, is given by

$$
\left[\|\mathscr{H}\|_{2}^{2}\right]\left(k_{z}\right)=f\left(k_{z}\right) R+g\left(k_{z}\right) R^{3},
$$

where $f:=f_{u x}+f_{v y}+f_{v z}+f_{w y}+f_{w z}=f_{x}+f_{y}+f_{z}=f_{u}+f_{v}+f_{w}$ and $g:=$ $g_{u y}+g_{u z}=g_{y}+g_{z}=g_{u}$.

As shown in Appendix E, the dependence of variance amplification on the Reynolds number at $k_{x}=0$ has the same form for the 'unstructured' and 'structured' external excitations. However, the expressions for functions $f$ and $g$ in Theorem 1 and Corollaries 2, 3 and 4 are different in these two cases.

In $\S 5$, we study the capability of the streamwise constant near-wall input components.

\section{Variance amplification of streamwise constant near-wall external excitations}

In this section, we study system (2.6) in the presence of streamwise constant threedimensional perturbations. By setting $k_{x}=0$ in (2.6) we obtain

$$
\begin{aligned}
\partial_{t}\left[\begin{array}{l}
\psi_{1} \\
\psi_{2}
\end{array}\right] & =\left[\begin{array}{cc}
\frac{1}{R} \mathscr{L} & 0 \\
\mathscr{C}_{p} & \frac{1}{R} \mathscr{S}
\end{array}\right]\left[\begin{array}{l}
\psi_{1} \\
\psi_{2}
\end{array}\right]+\left[\begin{array}{ccc}
0 & \mathscr{B}_{y 01} & \mathscr{B}_{z 01} \\
\mathscr{B}_{x 02} & 0 & 0
\end{array}\right]\left[\begin{array}{l}
d_{x 0} \\
d_{y 0} \\
d_{z 0}
\end{array}\right], \\
{\left[\begin{array}{l}
u \\
v \\
w
\end{array}\right] } & =\left[\begin{array}{cc}
0 & \mathscr{C}_{u 2} \\
\mathscr{C}_{v 1} & 0 \\
\mathscr{C}_{w 1} & 0
\end{array}\right]\left[\begin{array}{l}
\psi_{1} \\
\psi_{2}
\end{array}\right],
\end{aligned}
$$


with $\left\{\mathscr{B}_{x 02}:=\mathrm{i} k_{z} \kappa_{x}(y), \mathscr{B}_{y 01}:=-k_{z}^{2} \Delta^{-1} \kappa_{y}(y), \mathscr{B}_{z 01}:=-\mathrm{i} k_{z} \Delta^{-1}\left(\kappa_{z}^{\prime}(y)+\kappa_{z}(y) \partial_{y}\right)\right\}$, where $\kappa_{s}(y)$, for every $s=x, y, z$, denote arbitrary functions of $y$, and $\kappa_{z}^{\prime}(y):=\mathrm{d} \kappa_{z}(y) / \mathrm{d} y$. All other operators have the same meaning as in (4.1).

Since operators $\mathscr{A}, \mathscr{B}$ and $\mathscr{C}$ in (4.1) and (5.1) have the same respective structures, we conclude that all theorems and corollaries of $\S 4$ that determine scaling with $R$ of different $\mathscr{H}_{2}$ norms also hold for system (5.1). However, the expressions for the Reynolds-number-independent quantities are different for the 'unstructured' and 'structured' external excitations.

\subsection{Effectiveness of near-wall external excitations}

We next investigate the effectiveness of an input applied in a certain spatial direction by studying the Reynolds number dependence of $\left[\left\|\mathscr{H}_{s}\right\|_{2}^{2}\right]\left(k_{z}\right)$, where

$$
\mathscr{H}_{s}\left(k_{z}, \omega\right):=\mathscr{C}\left(k_{z}\right)\left(\mathrm{i} \omega \boldsymbol{I}-\mathscr{A}\left(k_{z}\right)\right)^{-1} \mathscr{B}_{s 0}\left(k_{z}\right) \quad(s=x, y, z) .
$$

Based on the above remarks, it follows that Corollary 2 holds for both 'unstructured' and 'structured' external excitations. However, the expressions for functions $f_{x}, f_{y}, f_{z}$, $g_{y}$ and $g_{z}$ are different in these two cases. This is illustrated in Appendix E.2.3 and Appendix E.2.4 where we derive analytical expressions for $f_{x}, f_{y}, f_{z}$ (which are the same for all channel flows) for

$$
\kappa_{s}(y):=a(1+\operatorname{coth}(a)) \mathrm{e}^{-a(y+1)}=: \kappa(y, a), \quad a>0, \quad \forall s=x, y, z,
$$

and determine the dependence of $g_{y}$ and $g_{z}$ on both $k_{z}$ and $a$ in terms of easily computable series in Couette flow. This particular 'pre-modulation' in $y$ is chosen to analyse the effectiveness of inputs whose amplitude decays exponentially away from the lower wall. Clearly, this rate of decay (i.e. degree of localization) can be adjusted by assigning different values to parameter $a$. We note that functions $f$ and $g$ in Corollary 2 now depend on both $k_{z}$ and 'modulation parameter' $a$, e.g. $f_{x}=f_{x}\left(k_{z}, a\right)$.

We have normalized $\kappa(y, a)$ so that

$$
\int_{-1}^{1} \kappa(y, a) \mathrm{d} y=2, \quad \forall a>0,
$$

which allows for comparison between 'structured' (in the limit as $a \rightarrow 0$ ) and 'unstructured' results. Clearly, in the 'unstructured' case there is no 'pre-modulation' in $y$, that is

$$
\kappa(y) \equiv 1 \Rightarrow \int_{-1}^{1} \kappa(y) \mathrm{d} y=2 .
$$

The formulae for functions $f_{x}\left(k_{z}, a\right), f_{y}\left(k_{z}, a\right)$ and $f_{z}\left(k_{z}, a\right)$ are determined in Appendix E.2.3. Furthermore, the traces of nominal-velocity-dependent operators are determined in terms of rapidly convergent series for Couette flow in Appendix E.2.4.

The analytical expression for $f_{x}\left(k_{z}, a\right)$ is given by (E 22). By taking the limit of $f_{x}\left(k_{z}, a\right)$ as $a \rightarrow 0$ we obtain (E 16) which corresponds to the value of $f_{x}\left(k_{z}\right)$ for 'unstructured' external excitation. Furthermore, as $k_{z} \rightarrow \infty$, we have

$$
\lim _{k_{z} \rightarrow \infty} f_{x}\left(k_{z}, a\right)=0, \quad \forall a \in(0, \infty),
$$

which implies that for any finite value of $a>0$, function $f_{x}\left(k_{z}, a\right)$ decays to zero for big enough values of $k_{z}$. On the other hand, a limit of $f_{x}\left(k_{z}, a\right)$ as $a \rightarrow \infty$ is determined by

$$
\lim _{a \rightarrow \infty} f_{x}\left(k_{z}, a\right)=\frac{1}{2}, \quad \forall k_{z} \in \mathbb{R},
$$



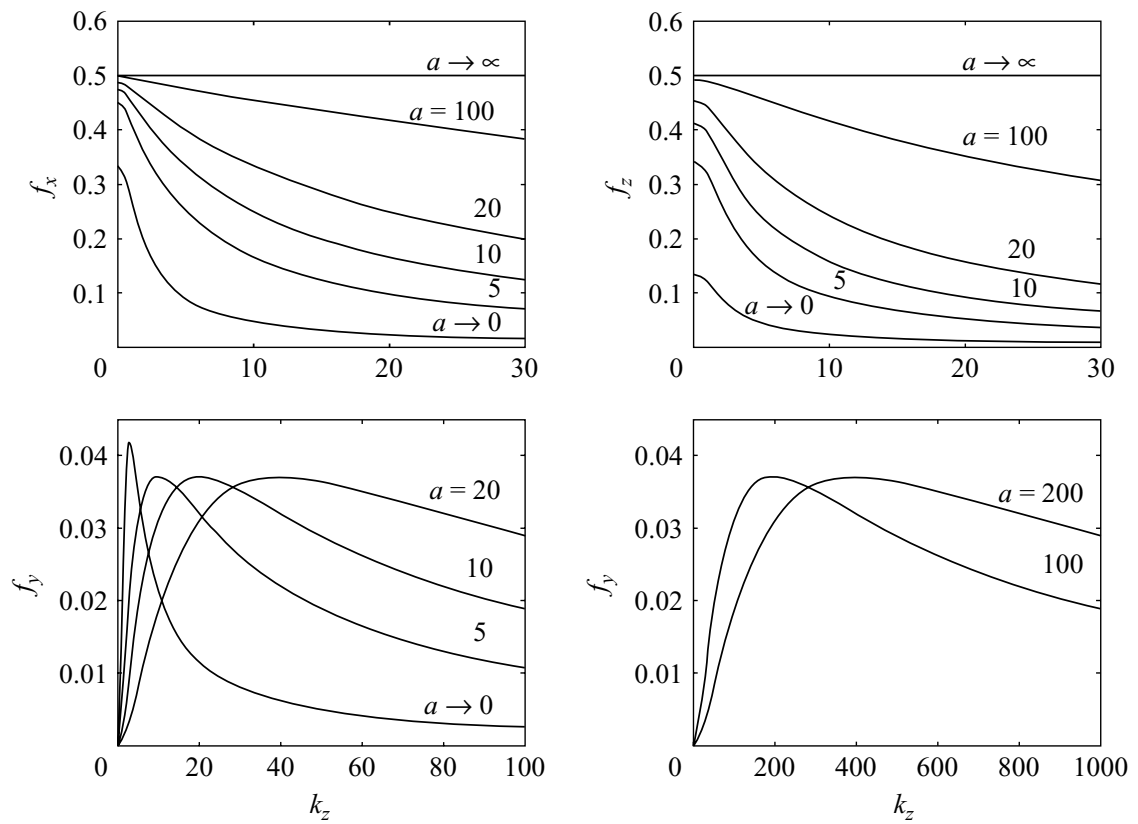

FIGURE 10. The $k_{z}$-dependence of $f_{x}, f_{y}$ and $f_{z}$ for different values of $a$.

which suggests that for external excitation that is localized in the vicinity of the lower wall (large enough values of $a$ ) and acts in the streamwise direction influence of high $k_{z}$ becomes increasingly important. This is illustrated in figure 10 where we graphically illustrate the dependence of $f_{x}$ on $k_{z}$ for different values of $a$.

The formulae for $f_{y}\left(k_{z}, a\right)$ and $f_{z}\left(k_{z}, a\right)$ are given by (E 23). The $k_{z}$-dependence of these two quantities for different values of $a$ is shown in figure 10 . We note that the limits of $f_{y}\left(k_{z}, a\right)$ and $f_{z}\left(k_{z}, a\right)$ as $a \rightarrow 0$ are in agreement with the corresponding formulae (E 17) for the 'unstructured' excitation.

The analytical expressions for $g_{y}\left(k_{z}, a\right)$ and $g_{z}\left(k_{z}, a\right)$ in Couette flow are determined in Appendix E.2.4. These formulae are expressed in terms of rapidly convergent series, and they are obtained by performing the spectral decompositions of the Orr-Sommerfeld and Squire operators. Since $R^{3}$ multiplies $g_{y}\left(k_{z}, a\right)$ and $g_{z}\left(k_{z}, a\right)$, respectively, in the expressions for $\left[\left\|\mathscr{H}_{y}\right\|_{2}^{2}\right]\left(k_{z}\right)$ and $\left[\left\|\mathscr{H}_{z}\right\|_{2}^{2}\right]\left(k_{z}\right)$ (see Corollary 2), it is relevant to analyse how $g_{y}\left(k_{z}, a\right)$ and $g_{z}\left(k_{z}, a\right)$ change with their arguments. Figure 11 shows the dependence of these two quantities on both $k_{z}$ and $a$. Clearly, the magnitude of $g_{y}$ decreases sharply as $a$ increases its value. On the other hand, the value of $g_{z}$ increases for a certain range of $a$ and then it starts decaying at a very slow rate. Furthermore, for any fixed value of parameter $a$, both $g_{y}$ and $g_{z}$ achieve largest values at $O(1)$ values of $k_{z}$.

The $a$-dependence of $\sup _{k_{z}} g_{y}\left(k_{z}, a\right), \sup _{k_{z}} g_{z}\left(k_{z}, a\right)$ and $\sup _{k_{z}} g_{z}\left(k_{z}, a\right) / \sup _{k_{z}} g_{y}\left(k_{z}, a\right)$ in Couette flow is illustrated in figure 12, with the abscissa in the linear and the ordinate in the logarithmic scale. Clearly, $\sup _{k} g_{y}\left(k_{z}, a\right)$ decays monotonically as a function of $a$ with a fairly sharp rate of decay, while $\sup _{k_{z}} g_{z}\left(k_{z}, a\right)$ peaks at a non-zero value of $a$ and then decays slowly as $a$ increases its value. Figure 12(c) further shows that $\sup _{k_{z}} g_{z}\left(k_{z}, a\right) / \sup _{k_{z}} g_{y}\left(k_{z}, a\right)$ is a monotonically increasing function of $a$ and that, for example, at $a=100$ function $\sup _{k_{z}} g_{z}\left(k_{z}, a\right)$ achieves almost four orders of magnitude larger value than function $\sup _{k_{z}} g_{y}\left(k_{z}, a\right)$. This indicates that the localized 

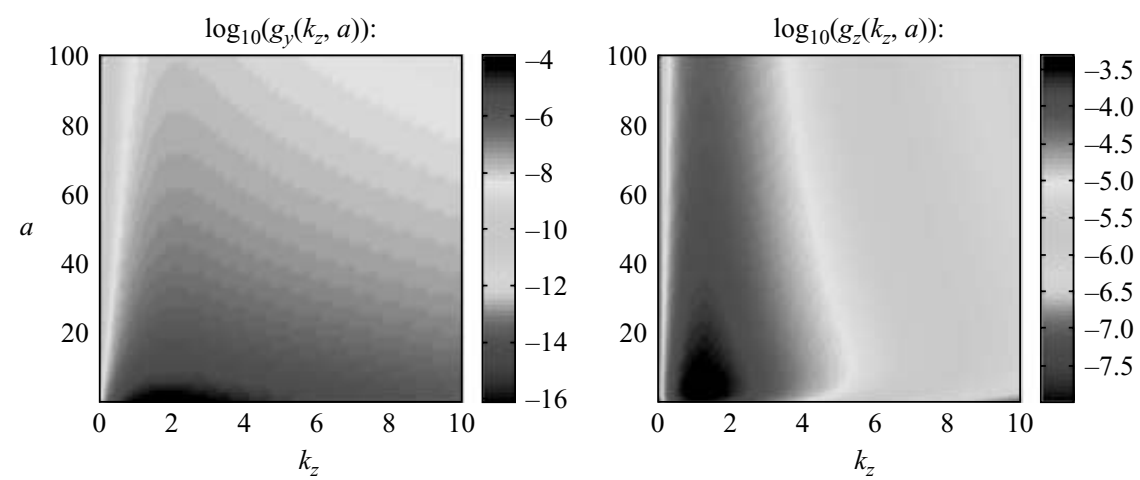

FIGURE 11. Plots of $g_{y}\left(k_{z}, a\right)$ and $g_{z}\left(k_{z}, a\right)$ in Couette flow.
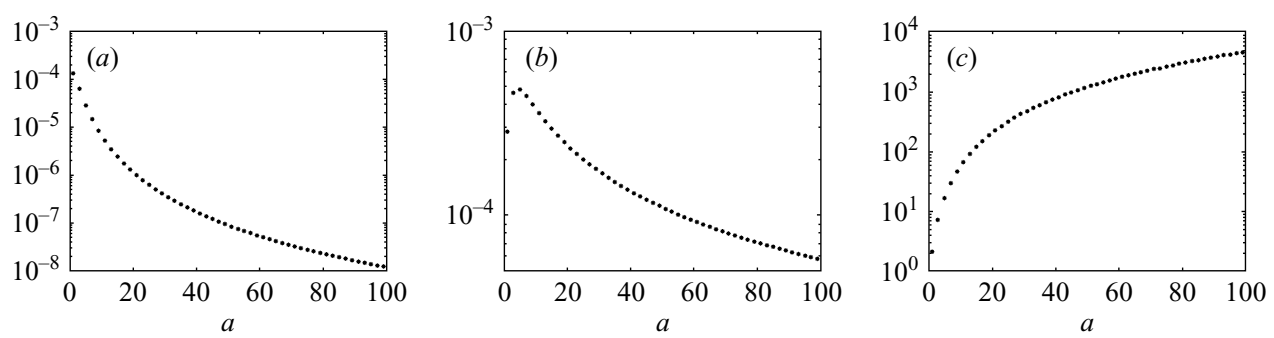

FIGURE 12. Plots of $(a) \sup _{k_{z}} g_{y}\left(k_{z}, a\right),(b) \sup _{k_{z}} g_{z}\left(k_{z}, a\right)$ and $(c) \sup _{k_{z}} g_{z}\left(k_{z}, a\right) / \sup _{k_{z}} g_{y}\left(k_{z}, a\right)$ in Couette flow.

spanwise excitations have much stronger influence on the velocity field than the localized wall-normal excitations. This observation is consistent with recent numerical work on channel-flow turbulence control using the Lorentz force (Berger et al. 2000; Du \& Karniadakis 2000; Du et al. 2002), where it was concluded that forcing in the spanwise direction confined within the viscous sub-layer had the strongest effect in suppressing turbulence. We confirmed this observation by analytical derivations for the streamwise constant perturbations showing that: for near-wall inputs, the spanwise forcing has, by far, the biggest impact on the evolution of the velocity field components.

\section{Concluding remarks}

We consider the NS equations linearized around some parallel channel flow $U(y)$, in the presence of spatially distributed and temporally varying three-dimensional body forces viewed as inputs. We carry out an input-output analysis in the frequency domain to investigate the dependence of different velocity 'outputs' $(u, v, w)$ on the different body force 'inputs' $\left(d_{x}, d_{y}, d_{z}\right)$. We demonstrate that this componentwise analysis uncovers several amplification mechanisms for subcritical transition, and show how the roles of TS waves, oblique waves and streamwise vortices and streaks can be explained as input-output resonances of the spatio-temporal frequency responses.

The effects of the inputs $\left(d_{x}, d_{y}, d_{z}\right)$ on velocity fields are dependent on various parameters. We perform a thorough study of these effects by quantifying them using system norms (also known as gains or amplifications), namely the so-called $\mathscr{H}_{\infty}$ and $\mathscr{H}_{2}$ norms. We focus on the latter quantity because it is more amenable to theoretical analysis in that we are able to obtain analytical expressions for the variances of 
streamwise constant perturbations. The numerical results for the $\mathscr{H}_{\infty}$ norm are qualitatively very similar to those for the $\mathscr{H}_{2}$ norm (Jovanovic 2004). Moreover, we investigate the dependence of the $\mathscr{H}_{2}$ norm on spatial frequencies, as well as the Reynolds number $R$. This frequency-response analysis yields several conclusions, one of which is the dominance of streamwise elongated-spanwise periodic $\left(k_{x} \approx 0\right.$, $\left.k_{z} \approx O(1)\right)$ and oblique $\left(k_{x} \approx O(1), k_{z} \approx O(1)\right)$ effects. We also perform a detailed analysis of the effect of individual inputs on individual outputs. We analytically ascertain that for 'channel-wide external excitations', the spanwise and wall-normal forces have the strongest influence on the velocity field; the impact of these forces is most powerful on the streamwise velocity component. For the streamwise constant perturbations, we rigorously establish that the square of the $\mathscr{H}_{2}$ norm from $d_{z}$ and $d_{y}$ to $u$ scales as $R^{3}$. On the other hand, at $k_{x}=0$, the $\mathscr{H}_{2}$ norms of operators from all other body force inputs to other velocity outputs scale at most as $R$. Furthermore, for 'near-wall excitations' we demonstrate that $d_{z}$ has, by far, the strongest effect on the evolution of the velocity field. This observation is consistent with recent numerical work on channel-flow turbulence control using the Lorentz force, where it was concluded that the spanwise forcing confined within the viscous sub-layer had the strongest effect in suppressing turbulence. We confirm this observation by analytical derivations that quantify the $\mathscr{H}_{2}$ norms of operators from individual inputs $\left(d_{x}, d_{y}\right.$ and $d_{z}$ ) to the entire velocity vector $\boldsymbol{\phi}$. Also, we calculate system norms as functions of spatial frequencies, and discuss the corresponding three-dimensional flow structures that are most amplified. These turn out to be typically streamwise elongated-spanwise periodic and sometimes oblique, which corresponds to structures commonly observed in experimental studies and fully nonlinear initial-value simulations in channel flows.

\section{Appendix A. The underlying operators}

The underlying Hilbert space for operator $\mathscr{A}$ is $\mathbb{H}_{\mathrm{OS}} \times L^{2}[-1,1]$, where (Reddy \& Henningson 1993)

$$
\mathbb{H}_{\mathrm{OS}}:=\left\{g \in L^{2}[-1,1] ; \quad g^{(2)} \in L^{2}[-1,1], g( \pm 1)=0\right\} .
$$

This operator is an unbounded operator defined on a domain $\mathscr{D}(\mathscr{A}):=\mathscr{D}\left(\mathscr{A}_{11}\right) \times$ $\mathscr{D}\left(\mathscr{A}_{22}\right)$. The domain of Squire operator $\mathscr{A}_{22}$ is equal to $\mathbb{H}_{\mathrm{OS}}$, and the domain of Orr-Sommerfeld operator $\mathscr{A}_{11}$ is defined by

$$
\mathscr{D}\left(\mathscr{A}_{11}\right):=\left\{g \in \mathbb{H}_{\mathrm{OS}} ; \quad g^{(4)} \in L^{2}[-1,1], g^{\prime}( \pm 1)=0\right\} .
$$

We endow the state-space $\mathbb{H}_{\mathrm{OS}} \times L^{2}[-1,1]$ with an inner product

$$
\left\langle\boldsymbol{\psi}_{1}, \boldsymbol{\psi}_{2}\right\rangle_{e}:=\left\langle\boldsymbol{\psi}_{1}, \mathscr{2} \boldsymbol{\psi}_{2}\right\rangle,
$$

where $\mathscr{2}$ is a block diagonal linear operator given by

$$
\mathscr{Z}:=\frac{1}{k_{x}^{2}+k_{z}^{2}}\left[\begin{array}{cc}
-\Delta & 0 \\
0 & I
\end{array}\right] \text {. }
$$

The inner product on the right-hand side of (A 1$)$ is the standard $L^{2}[-1,1]$ inner product. The inner product $\langle\cdot, \cdot\rangle_{e}$ determines then the kinetic energy density of a harmonic perturbation, which is a quadratic form of $v$ and $\omega_{y}$ as follows (Butler \& Farrell 1992)

$$
E=\langle\boldsymbol{\psi}, \boldsymbol{\psi}\rangle_{e}=\frac{1}{8} \int_{-1}^{1} \boldsymbol{\psi}^{*} \mathscr{Q} \boldsymbol{\psi} \mathrm{d} y=:\langle\boldsymbol{\psi}, \mathscr{Q} \boldsymbol{\psi}\rangle
$$


For the purposes of norm computations, it is important to identify the adjoints of the relevant operators. The adjoint of an operator $\mathscr{G}$ on a Hilbert space with an inner product $\langle\cdot, \cdot\rangle_{e}$, is defined by

$$
\left\langle\boldsymbol{\psi}_{1}, \mathscr{G}_{2}\right\rangle_{e}=\left\langle\mathscr{G}^{*} \boldsymbol{\psi}_{1}, \boldsymbol{\psi}_{2}\right\rangle_{e},
$$

which must hold for all $\boldsymbol{\psi}_{1}, \boldsymbol{\psi}_{2}$ in the Hilbert space $\mathbb{H}_{\mathrm{OS}} \times L^{2}[-1,1]$. $\mathscr{A}^{*}$ can be determined using (A 3 ), whereas the adjoints of the operators $\mathscr{B}$ and $\mathscr{C}$ are, respectively, given by

$$
\begin{aligned}
\langle\boldsymbol{\psi}, \mathscr{B} \boldsymbol{d}\rangle_{e} & =\left\langle\mathscr{B}^{*} \boldsymbol{\psi}, \boldsymbol{d}\right\rangle, \\
\langle\boldsymbol{\phi}, \mathscr{C} \boldsymbol{\psi}\rangle & =\left\langle\mathscr{C}^{*} \boldsymbol{\phi}, \boldsymbol{\psi}\right\rangle_{e} .
\end{aligned}
$$

The inner products on the right-hand side of (A $4 a)$ and the left-hand side of (A $4 b)$ are the standard $L^{2}[-1,1]$ inner products. Using (A 3), (A 4a) and (A $4 b$ ) we determine $\mathscr{A}^{*}, \mathscr{B}^{*}$, and $\mathscr{C}^{*}$ as

$$
\mathscr{A}^{*}=\left[\begin{array}{cc}
\mathscr{A}_{11}^{*} & \mathscr{A}_{21}^{*} \\
0 & \mathscr{A}_{22}^{*}
\end{array}\right], \quad \mathscr{B}^{*}=\left[\begin{array}{c}
\mathscr{B}_{x}^{*} \\
\mathscr{B}_{y}^{*} \\
\mathscr{B}_{z}^{*}
\end{array}\right], \quad \mathscr{C}^{*}=\left[\begin{array}{lll}
\mathscr{C}_{u}^{*} & \mathscr{C}_{v}^{*} & \mathscr{C}_{w}^{*}
\end{array}\right],
$$

where

$$
\begin{gathered}
\left\{\mathscr{A}_{11}^{*}=\mathrm{i} k_{x} U-\mathrm{i} k_{x} \Delta^{-1} U^{\prime \prime}+\frac{1}{R} \Delta^{-1} \Delta^{2}, \quad \mathscr{A}_{22}^{*}=\mathrm{i} k_{x} U+\frac{1}{R} \Delta, \quad \mathscr{A}_{21}^{*}=-\mathrm{i} k_{z} \Delta^{-1} U^{\prime}\right\}, \\
\left\{\mathscr{B}_{x}^{*}=\mathscr{C}_{u}, \quad \mathscr{B}_{y}^{*}=\mathscr{C}_{v}, \quad \mathscr{B}_{z}^{*}=\mathscr{C}_{w}\right\}, \quad\left\{\mathscr{C}_{u}^{*}=\mathscr{B}_{x}, \quad \mathscr{C}_{v}^{*}=\mathscr{B}_{y}, \quad \mathscr{C}_{w}^{*}=\mathscr{B}_{z}\right\} .
\end{gathered}
$$

We remark that

$$
\begin{aligned}
\mathscr{B}_{x} \mathscr{B}_{x}^{*}=\mathscr{C}_{u}^{*} \mathscr{C}_{u}=\frac{1}{k_{x}^{2}+k_{z}^{2}}\left[\begin{array}{cc}
k_{x}^{2} \Delta^{-1} \partial_{y y} & -k_{x} k_{z} \Delta^{-1} \partial_{y} \\
-k_{x} k_{z} \partial_{y} & k_{z}^{2}
\end{array}\right], \\
\mathscr{B}_{y} \mathscr{B}_{y}^{*}=\mathscr{C}_{v}^{*} \mathscr{C}_{v}=\left[\begin{array}{cc}
-\left(k_{x}^{2}+k_{z}^{2}\right) \Delta^{-1} & 0 \\
0 & 0
\end{array}\right], \\
\mathscr{B}_{z} \mathscr{B}_{z}^{*}=\mathscr{C}_{w}^{*} \mathscr{C}_{w}=\frac{1}{k_{x}^{2}+k_{z}^{2}}\left[\begin{array}{cc}
k_{z}^{2} \Delta^{-1} \partial_{y y} & k_{x} k_{z} \Delta^{-1} \partial_{y} \\
k_{x} k_{z} \partial_{y} & k_{x}^{2}
\end{array}\right],
\end{aligned}
$$

which in turn implies

$$
\mathscr{B} \mathscr{B}^{*}=\mathscr{C}^{*} \mathscr{C}=\left[\begin{array}{ll}
\boldsymbol{I} & 0 \\
0 & I
\end{array}\right] .
$$

For 'structured' external excitations (2.5) we have

$$
\begin{aligned}
& \mathscr{B}_{x 0} \mathscr{B}_{x 0}^{*}:=\mathscr{B}_{x} \kappa_{x}^{2} \mathscr{B}_{x}^{*}=\frac{1}{k_{x}^{2}+k_{z}^{2}}\left[\begin{array}{cc}
k_{x}^{2} \Delta^{-1}\left(2 \kappa_{x} \kappa_{x}^{\prime} \partial_{y}+\kappa_{x}^{2} \partial_{y y}\right) & -2 k_{x} k_{z} \Delta^{-1} \kappa_{x} \kappa_{x}^{\prime} \\
-k_{x} k_{z} \kappa_{x}^{2} \partial_{y} & k_{z}^{2} \kappa_{x}^{2}
\end{array}\right], \\
& \mathscr{B}_{y 0} \mathscr{B}_{y 0}^{*}:=\mathscr{B}_{y} \kappa_{y}^{2} \mathscr{B}_{y}^{*}=\left[\begin{array}{cc}
-\left(k_{x}^{2}+k_{z}^{2}\right) \Delta^{-1} \kappa_{y}^{2} & 0 \\
0 & 0
\end{array}\right], \\
& \mathscr{B}_{z 0} \mathscr{B}_{z 0}^{*}:=\mathscr{B}_{z} \kappa_{z}^{2} \mathscr{B}_{z}^{*}=\frac{1}{k_{x}^{2}+k_{z}^{2}}\left[\begin{array}{cc}
k_{z}^{2} \Delta^{-1}\left(2 \kappa_{z} \kappa_{z}^{\prime} \partial_{y}+\kappa_{z}^{2} \partial_{y y}\right) & 2 k_{x} k_{z} \Delta^{-1} \kappa_{z} \kappa_{z}^{\prime} \\
k_{x} k_{z} \kappa_{z}^{2} \partial_{y} & k_{x}^{2} \kappa_{z}^{2}
\end{array}\right],
\end{aligned}
$$


where $\kappa_{s}^{\prime}:=\mathrm{d} \kappa_{s}(y) / \mathrm{d} y$, for $s=x, y$ or $z$. The expressions (A 5)-(A 9) are used for norm computations of the spatio-temporal frequency responses.

\section{Appendix B. Spectral analysis of Squire and Orr-Sommerfeld operators at $k_{x}=0$}

We next perform spectral analysis of the Reynolds-number independent, normalized Squire and Orr-Sommerfeld operators at $k_{x}=0$. These two operators are denoted by $\mathscr{S}:=\Delta$ and $\mathscr{L}:=\Delta^{-1} \Delta^{2}$, and their domains are respectively determined by $\mathbb{H}_{\mathrm{OS}}$ and $\mathscr{D}\left(\mathscr{A}_{11}\right)$. At any fixed $k_{z}$ both $\mathscr{S}$ and $\mathscr{L}$ are self-adjoint (with respect to the inner products of $L^{2}[-1,1]$ and $\mathbb{H}_{\mathrm{OS}}$, respectively) with discrete spectra, and they are negative definite (with respect to the aforementioned inner products). Thus, these two operators have only negative eigenvalues and generate stable evolutions. We note that the $\mathbb{H}_{\mathrm{OS}}$ inner product is given by (Reddy \& Henningson 1993)

$$
\left\langle g_{1}, g_{2}\right\rangle_{o s}:=\left\langle g_{1}^{\prime}, g_{2}^{\prime}\right\rangle_{2}+k_{z}^{2}\left\langle g_{1}, g_{2}\right\rangle_{2}=-\left\langle g_{1}, \Delta g_{2}\right\rangle_{2} .
$$

It is well known that $\mathscr{S}$ has the following set of orthonormal eigenfunctions $\left\{\varphi_{n}\right\}_{n \in \mathbb{N}}$ with corresponding eigenvalues $\left\{\gamma_{n}\right\}_{n \in \mathbb{N}}$

$$
\varphi_{n}(y):=\sin \left(\frac{1}{2} n \pi(y+1)\right), \quad \gamma_{n}\left(k_{z}\right):=-\left(\frac{1}{4} n^{2} \pi^{2}+k_{z}^{2}\right), \quad n \in \mathbb{N} .
$$

The spectral analysis of $\mathscr{L}$ was performed by Dolph \& Lewis (1958). It was shown that $\mathscr{L}$ has the set of eigenfunctions $\left\{\sigma_{k}\right\}_{k \in \mathbb{N}}$

$$
\begin{aligned}
& \sigma_{k}\left(y, k_{z}, \lambda_{k}\right) \\
& \quad=A_{k}\left(\cos \left(p_{k} y\right)-\frac{\cos \left(p_{k}\right)}{\cosh \left(k_{z}\right)} \cosh \left(k_{z} y\right)\right)+B_{k}\left(\sin \left(p_{k} y\right)-\frac{\sin \left(p_{k}\right)}{\sinh \left(k_{z}\right)} \sinh \left(k_{z} y\right)\right) \\
& \quad=: \sigma_{1 k}\left(y, k_{z}, \lambda_{k}\right)+\sigma_{2 k}\left(y, k_{z}, \lambda_{k}\right),
\end{aligned}
$$

with corresponding eigenvalues $\left\{\lambda_{k}\right\}_{k \in \mathbb{N}}$

$$
\lambda_{k}:=-\left(p_{k}^{2}+k_{z}^{2}\right)
$$

where $p_{k}$ is obtained as a solution to either of the following two equations

$$
\begin{aligned}
& p_{k} \tan \left(p_{k}\right)=-k_{z} \tanh \left(k_{z}\right), \\
& p_{k} \cot \left(p_{k}\right)=k_{z} \operatorname{coth}\left(k_{z}\right) .
\end{aligned}
$$

It can be readily shown (Dolph \& Lewis 1958) that (B 1a) and (B 1b) cannot be satisfied simultaneously. In other words, if $\bar{p}_{k}$ is obtained as a solution to (B 1a) then $\bar{p}_{k}$ does not satisfy (B $1 b$ ), and vice versa. Furthermore, if (B $\left.1 a\right)$ is satisfied then $\left\{A_{k} \neq 0, B_{k} \equiv 0\right\}$, and if (B 1b) is satisfied then $\left\{A_{k} \equiv 0, B_{k} \neq 0\right\}$ (Dolph \& Lewis 1958), that is

$$
\begin{aligned}
& p_{k} \tan \left(p_{k}\right)=-k_{z} \tanh \left(k_{z}\right) \Rightarrow\left\{A_{k} \neq 0, \quad B_{k} \equiv 0\right\}, \\
& p_{k} \cot \left(p_{k}\right)=k_{z} \operatorname{coth}\left(k_{z}\right) \Rightarrow\left\{A_{k} \equiv 0, B_{k} \neq 0\right\} .
\end{aligned}
$$

This implies that we can consider separately the spectral decompositions of $\mathscr{L}$ in terms of $\left\{\sigma_{1 k}\right\}_{k \in \mathbb{N}}$ and $\left\{\sigma_{2 k}\right\}_{k \in \mathbb{N}}$ and combine them to determine the overall solution. 
In particular, the following choices of $\left\{A_{k}\right\}_{k \in \mathbb{N}}$ and $\left\{B_{k}\right\}_{k \in \mathbb{N}}$

$$
\begin{aligned}
& A_{k}:=\left\{\left(p_{1 k}^{2}+k_{z}^{2}\right)\left(1+\frac{\sin \left(2 p_{1 k}\right)}{2 p_{1 k}}\right)\right\}^{-1 / 2}, \quad B_{k} \equiv 0, \quad p_{1 k} \tan \left(p_{1 k}\right)=-k_{z} \tanh \left(k_{z}\right), \\
& A_{k} \equiv 0, \quad B_{k}:=\left\{\left(p_{2 k}^{2}+k_{z}^{2}\right)\left(1-\frac{\sin \left(2 p_{2 k}\right)}{2 p_{2 k}}\right)\right\}^{-1 / 2}, \quad p_{2 k} \cot \left(p_{2 k}\right)=k_{z} \operatorname{coth}\left(k_{z}\right),
\end{aligned}
$$

respectively, give the orthonormal sets of eigenfunctions $\left\{\sigma_{1 k}\right\}_{k \in \mathbb{N}}$ and $\left\{\sigma_{2 k}\right\}_{k \in \mathbb{N}}$.

\section{Appendix C. Numerical method}

In this section, we briefly describe the numerical method used for approximating the LNS equations. In the spatially invariant directions, frequency response calculations are done by 'gridding' in the wavenumber space $\left(k_{x}, k_{z}\right)$. In the wall-normal direction $y$, a Galerkin scheme (Boyd 1989) is used based on expansion using a set of basis functions that satisfy the appropriate boundary conditions (Leonard, personal communication 1999).

We denote the basis functions for wall-normal velocity $(v)$ and vorticity $\left(\omega_{y}\right)$ by $\alpha_{n}(y)$ and $\beta_{n}(y)$ respectively. They are defined in terms of Chebyshev polynomials (Boyd 1989) as follows:

$$
\alpha_{n}(y):=\left(1-y^{2}\right)^{2} T_{n}(y), \quad \beta_{n}(y):=\left(1-y^{2}\right) T_{n}(y) .
$$

Clearly, these basis functions satisfy boundary conditions given by (2.4). If we assume that the number of $v$ and $\omega_{y}$ basis functions is equal to $N$ and $M$, respectively, we can express $v$ and $\omega_{y}$ as

$$
v\left(k_{x}, y, k_{z}, t\right) \approx \sum_{n=0}^{N} a_{n}\left(k_{x}, k_{z}, t\right) \alpha_{n}(y), \quad \omega_{y}\left(k_{x}, y, k_{z}, t\right) \approx \sum_{n=0}^{M} b_{n}\left(k_{x}, k_{z}, t\right) \beta_{n}(y),
$$

where $a_{n}\left(k_{x}, k_{z}, t\right)$ and $b_{n}\left(k_{x}, k_{z}, t\right)$ are the so-called spectral coefficients. These coefficients are computed as a solution of a first order ODE obtained as a result of applying the Galerkin scheme to (2.2).

To obtain the corresponding ODE, we must find matrix representations of all operators in (2.2). We note that these matrix entries can be calculated without numerical integration by exploiting the recursive relations between Chebyshev polynomials and their derivatives (Boyd 1989). These matrix representations were developed for the case of a channel flow linearized around a nominal velocity profile of the form $\overline{\boldsymbol{u}}=\left[\begin{array}{lll}U(y) & 0 & 0\end{array}\right]^{T}$. All numerical computations in this paper were performed using MATLAB.

\section{Appendix D. Proof of Theorem 1}

From the block diagram representing the LNS system (4.1) at $k_{x}=0$ (see figure 6) it follows that

$$
\begin{aligned}
{\left[\begin{array}{c}
u \\
v \\
w
\end{array}\right]=} & {\left[\begin{array}{ccc}
\mathscr{H}_{u x}\left(k_{z}, \omega, R\right) & \mathscr{H}_{u y}\left(k_{z}, \omega, R\right) & \mathscr{H}_{u z}\left(k_{z}, \omega, R\right) \\
\mathscr{H}_{v x}\left(k_{z}, \omega, R\right) & \mathscr{H}_{v y}\left(k_{z}, \omega, R\right) & \mathscr{H}_{v z}\left(k_{z}, \omega, R\right) \\
\mathscr{H}_{w x}\left(k_{z}, \omega, R\right) & \mathscr{H}_{w y}\left(k_{z}, \omega, R\right) & \mathscr{H}_{w z}\left(k_{z}, \omega, R\right)
\end{array}\right]\left[\begin{array}{l}
d_{x} \\
d_{y} \\
d_{z}
\end{array}\right] } \\
:= & {\left[\begin{array}{ccc}
R \overline{\mathscr{H}}_{u x}\left(k_{z}, \Omega\right) & R^{2} \overline{\mathscr{H}}_{u y}\left(k_{z}, \Omega\right) & R^{2} \overline{\mathscr{H}}_{u z}\left(k_{z}, \Omega\right) \\
0 & R \overline{\mathscr{H}}_{v y}\left(k_{z}, \Omega\right) & R \overline{\mathscr{H}}_{v z}\left(k_{z}, \Omega\right) \\
0 & R \overline{\mathscr{H}}_{w y}\left(k_{z}, \Omega\right) & R \overline{\mathscr{H}}_{w z}\left(k_{z}, \Omega\right)
\end{array}\right]\left[\begin{array}{c}
d_{x} \\
d_{y} \\
d_{z}
\end{array}\right], }
\end{aligned}
$$


where $\Omega:=\omega R$, and

$$
\left\{\begin{aligned}
& \overline{\mathscr{H}}_{u x}\left(k_{z}, \Omega\right):=\mathscr{C}_{u 2}(\mathrm{i} \Omega \boldsymbol{I}-\mathscr{S})^{-1} \mathscr{B}_{x 2}, \\
& \overline{\mathscr{H}}_{u y}\left(k_{z}, \Omega\right):=\mathscr{C}_{u 2}(\mathrm{i} \Omega \boldsymbol{I}-\mathscr{S})^{-1} \mathscr{C}_{p}(\mathrm{i} \Omega \boldsymbol{I}-\mathscr{L})^{-1} \mathscr{B}_{y 1}, \\
& \overline{\mathscr{H}}_{u z}\left(k_{z}, \Omega\right):=\mathscr{C}_{u 2}(\mathrm{i} \Omega \boldsymbol{I}-\mathscr{S})^{-1} \mathscr{C}_{p}(\mathrm{i} \Omega \boldsymbol{I}-\mathscr{L})^{-1} \mathscr{B}_{z 1}, \\
& \overline{\mathscr{H}}_{r s}\left(k_{z}, \Omega\right):=\mathscr{C}_{r 1}(\mathrm{i} \Omega \boldsymbol{I}-\mathscr{L})^{-1} \mathscr{B}_{s 1}, \quad\{\boldsymbol{r}=v, w ; s=y, z\} .
\end{aligned}\right.
$$

For example, $\left[\left\|\mathscr{H}_{u y}\right\|_{2}^{2}\right]\left(k_{z}\right)$ is determined by

$$
\begin{aligned}
{\left[\left\|\mathscr{H}_{u y}\right\|_{2}^{2}\right]\left(k_{z}\right) } & :=\frac{1}{2 \pi} \int_{-\infty}^{\infty}\left\|\mathscr{H}_{u y}\left(k_{z}, \omega, R\right)\right\|_{H S}^{2} \mathrm{~d} \omega \\
& =\frac{1}{2 \pi} \int_{-\infty}^{\infty} \operatorname{trace}\left(\mathscr{H}_{u y}\left(k_{z}, \omega, R\right) \mathscr{H}_{u y}^{*}\left(k_{z}, \omega, R\right)\right) \mathrm{d} \omega \\
& =\frac{R^{4}}{2 \pi} \int_{-\infty}^{\infty} \operatorname{trace}\left(\overline{\mathscr{H}}_{u y}\left(k_{z}, \Omega\right) \overline{\mathscr{H}}_{u y}^{*}\left(k_{z}, \Omega\right)\right) \mathrm{d} \omega \\
& =\frac{R^{3}}{2 \pi} \int_{-\infty}^{\infty} \operatorname{trace}\left(\overline{\mathscr{H}}_{u y}\left(k_{z}, \Omega\right) \overline{\mathscr{H}}_{u y}^{*}\left(k_{z}, \Omega\right)\right) \mathrm{d} \Omega=: R^{3} g_{u y}\left(k_{z}\right) .
\end{aligned}
$$

A similar procedure can be used to determine the $\mathscr{H}_{2}$ norms of all other components of operator $\mathscr{H}$ in (2.12), which proves Theorem 1. Corollaries 2, 3 and 4 follow from Theorem 1 using the square-additivity of the $\mathscr{H}_{2}$ norm.

In Appendix E we show how functions $f$ and $g$ in (4.2) can be obtained based on solutions of the corresponding operator Lyapunov equations.

\section{Appendix E. Exact determination of $\mathscr{H}_{2}$ norms}

In $\S$ E.1, we derive expressions for the componentwise $\mathscr{H}_{2}$ norms of streamwise constant LNS system (4.1) in terms of solutions to the corresponding Lyapunov equations. In $\S$ E.2, we use these relationships to determine the explicit $k_{z}$-dependence of functions $f$ and $g$ in Corollaries 2 and 3 for both 'unstructured' and 'structured' external excitations. The nominal-flow-dependent functions $g$ are determined in Couette flow. The main analytical tool we use in $\S \mathrm{E} .2$ is the formula for the trace of a class of differential operators defined by forced two-point boundary-value state-space realizations (TPBVSR). This formula was derived by Jovanovic \& Bamieh (2005).

From $\S 4$ and Appendix $\mathrm{A}$ it follows that at $k_{x}=0$

$$
\begin{aligned}
& \mathscr{A}=\left[\begin{array}{cc}
\frac{1}{R} \Delta^{-1} \Delta^{2} & 0 \\
-\mathrm{i} k_{z} U^{\prime} & \frac{1}{R} \Delta
\end{array}\right]=:\left[\begin{array}{cc}
\frac{1}{R} \mathscr{L} & 0 \\
\mathscr{C}_{p} & \frac{1}{R} \mathscr{S}
\end{array}\right], \\
& \mathscr{A}^{*}=\left[\begin{array}{cc}
\frac{1}{R} \Delta^{-1} \Delta^{2} & -\mathrm{i} k_{z} \Delta^{-1} U^{\prime} \\
0 & \frac{1}{R} \Delta
\end{array}\right]=:\left[\begin{array}{cc}
\frac{1}{R} \mathscr{L} & \mathscr{C}_{p}^{*} \\
0 & \frac{1}{R} \mathscr{S}
\end{array}\right], \\
& \mathscr{M}_{x}=\mathscr{N}_{u}=\left[\begin{array}{ll}
0 & 0 \\
0 & \boldsymbol{I}
\end{array}\right], \quad \mathscr{M}_{y}=\mathscr{N}_{v}=\left[\begin{array}{cc}
-k_{z}^{2} \Delta^{-1} & 0 \\
0 & 0
\end{array}\right], \quad \mathscr{M}_{z}=\mathscr{N}_{w}=\left[\begin{array}{cc}
\Delta^{-1} \partial_{y y} & 0 \\
0 & 0
\end{array}\right],
\end{aligned}
$$

where operators $\mathscr{M}_{s}$ and $\mathscr{N}_{r}$ are defined as $\mathscr{M}_{s}:=\mathscr{B}_{s} \mathscr{B}_{s}^{*}$, for $s=x, y, z$, and $\mathscr{N}_{r}:=\mathscr{C}_{r}^{*} \mathscr{C}_{r}$, for $r=u, v, w$. The results presented here also hold for system (2.6) 
with 'structured' external excitations since operators

$$
\begin{aligned}
\mathscr{B}_{x 0} \mathscr{B}_{x 0}^{*} & =\left[\begin{array}{ll}
0 & 0 \\
0 & \kappa_{u}^{2}
\end{array}\right], \quad \mathscr{B}_{y 0} \mathscr{B}_{y 0}^{*}=\left[\begin{array}{cc}
-k_{z}^{2} \Delta^{-1} \kappa_{v}^{2} & 0 \\
0 & 0
\end{array}\right], \\
\mathscr{B}_{z 0} \mathscr{B}_{z 0}^{*} & =\left[\begin{array}{cc}
\Delta^{-1}\left(2 \kappa_{w} \kappa_{w}^{\prime} \partial_{y}+\kappa_{w}^{2} \partial_{y y}\right) & 0 \\
0 & 0
\end{array}\right],
\end{aligned}
$$

have the same respective structures as operators $\mathscr{M}_{x}, \mathscr{M}_{y}$ and $\mathscr{M}_{z}$. As remarked in $\S 5$, the dependence of the variance amplification on the Reynolds number at $k_{x}=0$ has the same form for the 'unstructured' and 'structured' external excitations. However, the expressions for functions $f$ and $g$ in Theorem 1, Corollary 2, Corollary 3 and Corollary 4 are different in these two cases.

\section{E.1. Expressions for $\mathscr{H}_{2}$ norms via Lyapunov equations}

In this subsection, we provide alternative proofs of Theorem 1, Corollary 2 and Corollary 3 by exploiting the fact that the $\mathscr{H}_{2}$ norms of frequency response operators $\mathscr{H}_{r s}, \mathscr{H}_{s}$ and $\mathscr{H}_{r},\{r=u, v, w ; s=x, y, z\}$, can be determined based on solutions of the corresponding Lyapunov equations. The procedure outlined below is most convenient for determination of the Reynolds number independent functions $f$ and $g$ in (4.2), (4.3) and (4.4).

\section{E.1.1. Controllability Gramians and functions $f$ and $g$ in Theorem 1}

For each $r$ and $s$, [ $\left[\mathscr{H}_{r s} \|_{2}^{2}\right]\left(k_{z}\right)$ can be expressed in terms of the solution to an operator Lyapunov equation

$$
\mathscr{A} \mathscr{X}_{s}+\mathscr{X}_{s} \mathscr{A}^{*}=-\mathscr{M}_{s}
$$

as $\left[\left\|\mathscr{H}_{r s}\right\|_{2}^{2}\right]\left(k_{z}\right)=\operatorname{trace}\left(\mathscr{N}_{r} \mathscr{X}_{s}\right)$, where $\mathscr{X}_{s}$ is a $2 \times 2$ block operator whose structure we denote by

$$
\mathscr{X}_{s}:=\left[\begin{array}{ll}
\mathscr{X}_{s 11} & \mathscr{X}_{s 0}^{*} \\
\mathscr{X}_{s 0} & \mathscr{X}_{s 22}
\end{array}\right] .
$$

Using the structure of operators $\mathscr{N}_{r}$ and $\mathscr{X}_{s}$ it follows that $\left[\left\|\mathscr{H}_{r s}\right\|_{2}^{2}\right]\left(k_{z}\right)$ can be determined as

$$
\left[\left\|\mathscr{H}_{r s}\right\|_{2}^{2}\right]\left(k_{z}\right)=\operatorname{trace}\left(\mathscr{N}_{r} \mathscr{X}_{s}\right)=\operatorname{trace}\left(\mathscr{N}_{r 11} \mathscr{X}_{s 11}\right)+\operatorname{trace}\left(\mathscr{N}_{r 22} \mathscr{X}_{s 22}\right) .
$$

The lower block triangular structure of $\mathscr{A}$ transforms (E 1) into the following set of conveniently coupled operator Sylvester equations

$$
\begin{aligned}
\mathscr{L} \mathscr{X}_{s 11}+\mathscr{X}_{s 11} \mathscr{L} & =-R \mathscr{M}_{s 11}, \\
\mathscr{S} \mathscr{X}_{s 0}+\mathscr{X}_{s 0} \mathscr{L} & =-R \mathscr{C}_{p} \mathscr{X}_{s 11}, \\
\mathscr{S} \mathscr{X}_{s 22}+\mathscr{X}_{s 22} \mathscr{S} & =-R\left(\mathscr{M}_{s 22}+\mathscr{C}_{p} \mathscr{X}_{s 0}^{*}+\mathscr{X}_{s 0} \mathscr{C}_{p}^{*}\right) .
\end{aligned}
$$

If the solutions of this system of equations at $R=1$ are respectively denoted by $\mathscr{P}_{s 11}$, $\mathscr{P}_{s 0}$, and $\mathscr{P}_{s 22}$, then (E 2$)$ simplifies to

$$
\begin{aligned}
\mathscr{L} \mathscr{P}_{s 11}+\mathscr{P}_{s 11} \mathscr{L} & =-\mathscr{M}_{s 11}, \\
\mathscr{S} \mathscr{P}_{s 0}+\mathscr{P}_{s 0} \mathscr{L} & =-\mathscr{C}_{p} \mathscr{P}_{s 11}, \\
\mathscr{S} \mathscr{P}_{s 22_{2}}+\mathscr{P}_{s 22} \mathscr{S} & =-\left(\mathscr{C}_{p} \mathscr{P}_{s 0}^{*}+\mathscr{P}_{s 0} \mathscr{C}_{p}^{*}\right), \\
\mathscr{S} \mathscr{P}_{s 22_{1}}+\mathscr{P}_{s 22_{1}} \mathscr{S} & =-\mathscr{M}_{s 22},
\end{aligned}
$$


where $\mathscr{P}_{s 22}=\mathscr{P}_{s 22_{1}}+\mathscr{P}_{s 22_{2}}$. Clearly, (E $\left.3 a\right)$ and (E 3d) can be respectively solved for $\mathscr{P}_{s 11}$ and $\mathscr{P}_{s 22_{1}}$. Once $\mathscr{P}_{s 11}$ is determined, $(\mathrm{E} 3 b)$ can be solved to yield $\mathscr{P}_{s 0}$. Finally, $\mathscr{P}_{s 0}$ can be used to determine solution to (E $\left.3 c\right)$.

Linearity of (E 2) implies that its solutions can be expressed in terms of solutions to (E 3) as

$$
\left\{\mathscr{X}_{s 11}=R \mathscr{P}_{s 11}, \quad \mathscr{X}_{s 0}=R^{2} \mathscr{P}_{s 0}, \quad \mathscr{X}_{s 22}=R \mathscr{P}_{s 22_{1}}+R^{3} \mathscr{P}_{s 22_{2}}\right\},
$$

which yields

$$
\left[\left\|\mathscr{H}_{r s}\right\|_{2}^{2}\right]\left(k_{z}\right)=\left(\operatorname{trace}\left(\mathscr{N}_{r 11} \mathscr{P}_{s 11}\right)+\operatorname{trace}\left(\mathscr{N}_{r 22} \mathscr{P}_{s 22_{1}}\right)\right) R+\operatorname{trace}\left(\mathscr{N}_{r 22} \mathscr{P}_{s 22_{2}}\right) R^{3} .
$$

Using the definitions of operators $\mathscr{N}_{r}$ and $\mathscr{M}_{s}$, it follows that

$$
\left\{\mathscr{N}_{u 11} \equiv 0, \quad \mathscr{N}_{v 22} \equiv 0, \quad \mathscr{N}_{w 22} \equiv 0\right\}, \quad\left\{\begin{array}{ll}
\mathscr{P}_{x 11} \equiv 0, & \mathscr{P}_{x 22_{2}} \equiv 0 \\
\mathscr{P}_{y 22_{1}} \equiv 0, & \mathscr{P}_{z 22_{1}} \equiv 0
\end{array}\right\},
$$

which in combination with (E 4 ) gives the following expressions for functions $f$ and $g$ in (4.2)

$$
\begin{aligned}
& {\left[\begin{array}{ccc}
f_{u x}\left(k_{z}\right) & g_{u y}\left(k_{z}\right) & g_{u z}\left(k_{z}\right) \\
0 & f_{v y}\left(k_{z}\right) & f_{v z}\left(k_{z}\right) \\
0 & f_{w y}\left(k_{z}\right) & f_{w z}\left(k_{z}\right)
\end{array}\right]} \\
& \quad=\left[\begin{array}{ccc}
\operatorname{trace}\left(\mathscr{N}_{u 22}\left(k_{z}\right) \mathscr{P}_{x 22_{1}}\left(k_{z}\right)\right) & \operatorname{trace}\left(\mathscr{N}_{u 22}\left(k_{z}\right) \mathscr{P}_{y 22_{2}}\left(k_{z}\right)\right) & \operatorname{trace}\left(\mathscr{N}_{u 22}\left(k_{z}\right) \mathscr{P}_{z 22_{2}}\left(k_{z}\right)\right) \\
0 & \operatorname{trace}\left(\mathscr{N}_{v 11}\left(k_{z}\right) \mathscr{P}_{y 11}\left(k_{z}\right)\right) & \operatorname{trace}\left(\mathscr{N}_{v 11}\left(k_{z}\right) \mathscr{P}_{z 11}\left(k_{z}\right)\right) \\
0 & \operatorname{trace}\left(\mathscr{N}_{w 11}\left(k_{z}\right) \mathscr{P}_{y 11}\left(k_{z}\right)\right) & \operatorname{trace}\left(\mathscr{N}_{w 11}\left(k_{z}\right) \mathscr{P}_{z 11}\left(k_{z}\right)\right)
\end{array}\right] .
\end{aligned}
$$

These expressions are most convenient for determination of functions $f$ and $g$ in Corollary 2.

E.1.2. Observability Gramians and functions $f$ and $g$ in Theorem 1

Alternatively, $\left[\left\|\mathscr{H}_{r s}\right\|_{2}^{2}\right]\left(k_{z}\right)$ can be, for every $\{r=u, v, w ; s=x, y, z\}$, expressed in terms of the solution to an operator Lyapunov equation of the form

$$
\mathscr{A}^{*} \mathscr{Y}_{r}+\mathscr{Y}_{r} \mathscr{A}=-\mathscr{N}_{r}
$$

as $\left[\left\|\mathscr{H}_{r s}\right\|_{2}^{2}\right]\left(k_{z}\right)=\operatorname{trace}\left(\mathscr{Y}_{r} \mathscr{M}_{s}\right)$, where $\mathscr{Y}_{r}$ is a $2 \times 2$ block operator whose structure we denote by

$$
\mathscr{Y}_{r}:=\left[\begin{array}{ll}
\mathscr{Y}_{r 11} & \mathscr{Y}_{r 0}^{*} \\
\mathscr{Y}_{r 0} & \mathscr{Y}_{r 22}
\end{array}\right]
$$

Using the structure of operators $\mathscr{M}_{s}$ and $\mathscr{Y}_{r}$ it follows that $\left[\left\|\mathscr{H}_{r s}\right\|_{2}^{2}\right]\left(k_{z}\right)$ can be determined as

$$
\left[\left\|\mathscr{H}_{r s}\right\|_{2}^{2}\right]\left(k_{z}\right)=\operatorname{trace}\left(\mathscr{Y}_{r} \mathscr{M}_{s}\right)=\operatorname{trace}\left(\mathscr{Y}_{r 11} \mathscr{M}_{s 11}\right)+\operatorname{trace}\left(\mathscr{Y}_{r 22} \mathscr{M}_{s 22}\right) .
$$

The lower block triangular structure of $\mathscr{A}$ renders (E 6) into the following set of conveniently coupled operator Sylvester equations

$$
\begin{aligned}
\mathscr{S} \mathscr{Y}_{r 22}+\mathscr{Y}_{r 22} \mathscr{S} & =-R \mathscr{N}_{r 22}, \\
\mathscr{S} \mathscr{Y}_{r 0}+\mathscr{Y}_{r 0} \mathscr{L} & =-R \mathscr{Y}_{r 22} \mathscr{C}_{p}, \\
\mathscr{L} \mathscr{Y}_{r 11}+\mathscr{Y}_{r 11} \mathscr{L} & =-R\left(\mathscr{N}_{r 11}+\mathscr{C}_{p}^{*} \mathscr{Y}_{r 0}+\mathscr{Y}_{r 0}^{*} \mathscr{C}_{p}\right) .
\end{aligned}
$$


If the solutions of this system of equations at $R=1$ are respectively denoted by $\mathscr{R}_{r 22}$, $\mathscr{R}_{r 0}$ and $\mathscr{R}_{r 11}$, then (E 7) simplifies to

$$
\begin{aligned}
\mathscr{S} \mathscr{R}_{r 22}+\mathscr{R}_{r 22} \mathscr{S} & =-\mathscr{N}_{r 22}, \\
\mathscr{S} \mathscr{R}_{r 0}+\mathscr{R}_{r 0} \mathscr{L} & =-\mathscr{R}_{r 22} \mathscr{C}_{p}, \\
\mathscr{L} \mathscr{R}_{r 11_{2}}+\mathscr{R}_{r 11_{2}} \mathscr{L} & =-\left(\mathscr{C}_{p}^{*} \mathscr{R}_{r 0}+\mathscr{R}_{r 0}^{*} \mathscr{C}_{p}\right), \\
\mathscr{L} \mathscr{R}_{r 11_{1}}+\mathscr{R}_{r 11_{1}} \mathscr{L} & =-\mathscr{N}_{r 11},
\end{aligned}
$$

where $\mathscr{R}_{r 11}=\mathscr{R}_{r 11_{1}}+\mathscr{R}_{r 11_{2}}$. Clearly, (E $\left.8 a\right)$ and (E $8 d$ ) can be respectively solved for $\mathscr{R}_{r 22}$ and $\mathscr{R}_{r 11}$. Once $\mathscr{R}_{r 22}$ is determined, (E $\left.8 b\right)$ can be solved to yield $\mathscr{R}_{r 0}$. Finally, $\mathscr{R}_{r 0}$ can be used to determine the solution to (E $8 c$ ).

Linearity of (E 7) implies that its solutions can be expressed in terms of solutions to $(\mathrm{E} 8)$ as

$$
\left\{\mathscr{Y}_{r 22}=R \mathscr{R}_{r 22}, \quad \mathscr{Y}_{r 0}=R^{2} \mathscr{R}_{r 0}, \quad \mathscr{Y}_{r 11}=R \mathscr{R}_{r 11_{1}}+R^{3} \mathscr{R}_{r 11_{2}}\right\},
$$

which yields

$$
\left[\left\|\mathscr{H}_{r s}\right\|_{2}^{2}\right]\left(k_{z}\right)=\left(\operatorname{trace}\left(\mathscr{R}_{r 22} \mathscr{M}_{s 22}\right)+\operatorname{trace}\left(\mathscr{R}_{r 11_{1}} \mathscr{M}_{s 11}\right)\right) R+\operatorname{trace}\left(\mathscr{R}_{r 11_{2}} \mathscr{M}_{s 11}\right) R^{3} .
$$

Using the definitions of operators $\mathscr{M}_{s}$, and $\mathscr{N}_{r}$, it follows that

$$
\left\{\begin{aligned}
&\left\{\mathscr{M}_{x 11} \equiv 0,\right.\left.\mathscr{M}_{y 22} \equiv 0, \quad \mathscr{M}_{z 22} \equiv 0\right\}, \\
&\left\{\mathscr{R}_{u 11_{1}} \equiv 0, \quad\left\{\mathscr{R}_{v 22} \equiv 0, \mathscr{R}_{v 11_{2}} \equiv 0\right\}, \quad\left\{\mathscr{R}_{w 22} \equiv 0, \mathscr{R}_{w 11_{2}} \equiv 0\right\}\right\},
\end{aligned}\right.
$$

which in combination with (E9) gives the following expressions for functions $f$ and $g$ in (4.2)

$$
\begin{aligned}
& {\left[\begin{array}{ccc}
f_{u x}\left(k_{z}\right) & g_{u y}\left(k_{z}\right) & g_{u z}\left(k_{z}\right) \\
0 & f_{v y}\left(k_{z}\right) & f_{v z}\left(k_{z}\right) \\
0 & f_{w y}\left(k_{z}\right) & f_{w z}\left(k_{z}\right)
\end{array}\right]} \\
& \quad=\left[\begin{array}{ccc}
\operatorname{trace}\left(\mathscr{R}_{u 22}\left(k_{z}\right) \mathscr{M}_{x 22}\left(k_{z}\right)\right) & \operatorname{trace}\left(\mathscr{R}_{u 11_{2}}\left(k_{z}\right) \mathscr{M}_{y 11}\left(k_{z}\right)\right) & \operatorname{trace}\left(\mathscr{R}_{u 11_{2}}\left(k_{z}\right) \mathscr{M}_{z 11}\left(k_{z}\right)\right) \\
0 & \operatorname{trace}\left(\mathscr{R}_{v 11_{1}}\left(k_{z}\right) \mathscr{M}_{y 11}\left(k_{z}\right)\right) & \operatorname{trace}\left(\mathscr{R}_{v 11_{1}}\left(k_{z}\right) \mathscr{M}_{z 11}\left(k_{z}\right)\right) \\
0 & \operatorname{trace}\left(\mathscr{R}_{w 11_{1}}\left(k_{z}\right) \mathscr{M}_{y 11}\left(k_{z}\right)\right) & \operatorname{trace}\left(\mathscr{R}_{w 11_{1}}\left(k_{z}\right) \mathscr{M}_{z 11}\left(k_{z}\right)\right)
\end{array}\right] .
\end{aligned}
$$

These expressions are most convenient for determination of functions $f$ and $g$ in Corollary 3.

\section{E.1.3. Expressions for functions $f$ and $g$ in Corollary 2 via Lyapunov equations}

Corollary 2 follows from Theorem 1 and square additivity of the $\mathscr{H}_{2}$ norm. Thus, for every $s=x, y, z$, the $\mathscr{H}_{2}$ norm of operator $\mathscr{H}_{s}$ from $d_{s}$ to $\phi:=\left[\begin{array}{ll}u & v\end{array}\right]^{T}$ is given by

$$
\left[\left\|\mathscr{H}_{s}\right\|_{2}^{2}\right]\left(k_{z}\right)=\left[\left\|\mathscr{H}_{u s}\right\|_{2}^{2}\right]\left(k_{z}\right)+\left[\left\|\mathscr{H}_{v s}\right\|_{2}^{2}\right]\left(k_{z}\right)+\left[\left\|\mathscr{H}_{w s}\right\|_{2}^{2}\right]\left(k_{z}\right) .
$$

Therefore, combination of (E 5) and (E 11) with $\mathscr{N}_{u 22}=\mathscr{N}_{v 11}+\mathscr{N}_{w 11}=\boldsymbol{I}$ yields

$$
\begin{aligned}
& {\left[\left\|\mathscr{H}_{x}\right\|_{2}^{2}\right]\left(k_{z}\right)=\operatorname{trace}\left(\mathscr{P}_{x 22_{1}}\left(k_{z}\right)\right) R \quad=: f_{x}\left(k_{z}\right) R,} \\
& {\left[\left\|\mathscr{H}_{y}\right\|_{2}^{2}\right]\left(k_{z}\right)=\operatorname{trace}\left(\mathscr{P}_{y 11}\left(k_{z}\right)\right) R+\operatorname{trace}\left(\mathscr{P}_{y 22_{2}}\left(k_{z}\right)\right) R^{3}=: f_{y}\left(k_{z}\right) R+g_{y}\left(k_{z}\right) R^{3},} \\
& {\left[\left\|\mathscr{H}_{z}\right\|_{2}^{2}\right]\left(k_{z}\right)=\operatorname{trace}\left(\mathscr{P}_{z 11}\left(k_{z}\right)\right) R+\operatorname{trace}\left(\mathscr{P}_{z 22_{2}}\left(k_{z}\right)\right) R^{3}=: f_{z}\left(k_{z}\right) R+g_{z}\left(k_{z}\right) R^{3},}
\end{aligned}
$$

which provides an alternative proof of Corollary 2, and gives convenient expressions for functions $f_{x}, f_{y}, f_{z}, g_{y}, g_{z}$ in terms of solutions to the corresponding 
Lyapunov equations. In $\S$ E.2, we combine these expressions with the trace formula of Jovanović \& Bamieh (2005) to derive formulae for $\left\{f_{x}\left(k_{z}\right), f_{y}\left(k_{z}\right), f_{z}\left(k_{z}\right), g_{y}\left(k_{z}\right)\right.$, $\left.g_{z}\left(k_{z}\right)\right\}$ for LNS systems (4.1) and (5.1) with 'unstructured' and 'structured' external excitations. Functions $g_{y}$ and $g_{z}$ are determined for Couette flow.

\section{E.1.4. Expressions for functions $f$ and $g$ in Corollary 3 via Lyapunov equations}

Corollary 3 follows from Theorem 1 and square additivity of the $\mathscr{H}_{2}$ norm. Thus, for every $r=u, v, w$, the $\mathscr{H}_{2}$ norm of operator $\mathscr{H}_{r}$ from $\boldsymbol{d}:=\left[\begin{array}{lll}d_{x} & d_{y} & d_{z}\end{array}\right]^{T}$ to $r$ is given by

$$
\left[\left\|\mathscr{H}_{r}\right\|_{2}^{2}\right]\left(k_{z}\right)=\left[\left\|\mathscr{H}_{r x}\right\|_{2}^{2}\right]\left(k_{z}\right)+\left[\left\|\mathscr{H}_{r y}\right\|_{2}^{2}\right]\left(k_{z}\right)+\left[\left\|\mathscr{H}_{r z}\right\|_{2}^{2}\right]\left(k_{z}\right) .
$$

Therefore, combination of (E 10) and (E 12) with $\mathscr{M}_{x 22}=\mathscr{M}_{y 11}+\mathscr{M}_{z 11}=\boldsymbol{I}$, yields

$$
\begin{array}{ll}
{\left[\left\|\mathscr{H}_{u}\right\|_{2}^{2}\right]\left(k_{z}\right)=\operatorname{trace}\left(\mathscr{R}_{u 22}\left(k_{z}\right)\right) R+\operatorname{trace}\left(\mathscr{R}_{u 11_{2}}\left(k_{z}\right)\right) R^{3}} & =: f_{u}\left(k_{z}\right) R+g_{u}\left(k_{z}\right) R^{3}, \\
{\left[\left\|\mathscr{H}_{v}\right\|_{2}^{2}\right]\left(k_{z}\right)=\operatorname{trace}\left(\mathscr{R}_{v 11_{1}}\left(k_{z}\right)\right) R} & =: f_{v}\left(k_{z}\right) R, \\
{\left[\left\|\mathscr{H}_{w}\right\|_{2}^{2}\right]\left(k_{z}\right)=\operatorname{trace}\left(\mathscr{R}_{w 11_{1}}\left(k_{z}\right)\right) R} & =: f_{w}\left(k_{z}\right) R,
\end{array}
$$

which provides an alternative proof of Corollary 3, and gives convenient expressions for functions $f_{u}, f_{v}, f_{w}$ and $g_{u}$ in terms of solutions to the corresponding Lyapunov equations. In $\S$ E.2, we combine these expressions with the trace formula of Jovanović \& Bamieh (2005) to derive formulae for $\left\{f_{u}\left(k_{z}\right), f_{v}\left(k_{z}\right), f_{w}\left(k_{z}\right), g_{u}\left(k_{z}\right)\right\}$ for LNS system (4.1) with 'unstructured' external excitations. Function $g_{u}$ is determined for Couette flow.

\section{E.2. Formulae for operator traces}

In this subsection, we derive analytical formulae for traces of operators that appear in the expressions for $\left[\left\|\mathscr{H}_{s}\right\|_{2}^{2}\right]\left(k_{z}\right),\{s=x, y, z\}$, and $\left[\left\|\mathscr{H}_{r}\right\|_{2}^{2}\right]\left(k_{z}\right),\{r=u, v, w\}$. The main analytical tool we use here is the formula for the trace of a class of differential operators defined by forced TPBVSR. This formula was derived by Jovanovic \& Bamieh (2005). In the process, we exploit the fact that some of the traces of interest were already determined by Bamieh \& Dahleh (2001).

Unstructured external excitations: determination of nominal velocity independent traces For system (4.1) with 'unstructured' external excitations, the nominal velocity independent operators (whose traces we want to determine) can be represented by a well-posed TPBVSR with constant coefficients in $y$

$$
\mathscr{F}:\left\{\begin{aligned}
x^{\prime}(y) & =A x(y)+B f(y), \\
g(y) & =C x(y), \\
0 & =L_{1} x(-1)+L_{2} x(1), \quad y \in[-1,1] .
\end{aligned}\right.
$$

Thus, the traces of these operators can be obtained using the trace formula of Jovanovic \& Bamieh (2005) as

$$
\operatorname{trace}(\mathscr{F})=\operatorname{tr}\left(C B-\left(L_{1}+L_{2} \mathrm{e}^{2 A}\right)^{-1} L_{2}\left[\begin{array}{ll}
0 & \boldsymbol{I}
\end{array}\right] \exp \left\{2\left[\begin{array}{cc}
A & 0 \\
B C & A
\end{array}\right]\right\}\left[\begin{array}{l}
\boldsymbol{I} \\
0
\end{array}\right]\right)
$$

This formula is employed to determine explicit analytical expressions for functions $f_{r}\left(k_{z}\right),\{r=u, v, w\}$, and $f_{s}\left(k_{z}\right),\{s=x, y, z\}$ in Corollaries 2 and 3. 
E.2.1. Formulae for $f_{x}\left(k_{z}\right), f_{y}\left(k_{z}\right)$ and $f_{z}\left(k_{z}\right)$

We recall that $f_{x}\left(k_{z}\right), f_{y}\left(k_{z}\right)$ and $f_{z}\left(k_{z}\right)$ are respectively determined by $\operatorname{trace}\left(\mathscr{P}_{x 22_{1}}\left(k_{z}\right)\right)$, trace $\left(\mathscr{P}_{y 11}\left(k_{z}\right)\right)$ and trace $\left(\mathscr{P}_{z 11}\left(k_{z}\right)\right)$, where operators $\mathscr{P}_{x 22_{1}}, \mathscr{P}_{y 11}$ and $\mathscr{P}_{z 11}$ satisfy

$$
\begin{aligned}
\mathscr{S} \mathscr{P}_{x 22_{1}}+\mathscr{P}_{x 22_{1}} \mathscr{S} & =-\boldsymbol{I}, \\
\mathscr{L} \mathscr{P}_{y 11}+\mathscr{P}_{y 11} \mathscr{L} & =-\left(-k_{z}^{2} \Delta^{-1}\right), \\
\mathscr{L} \mathscr{P}_{z 11}+\mathscr{P}_{z 11} \mathscr{L} & =-\Delta^{-1} \partial_{y y} .
\end{aligned}
$$

The solution of (E 15a) is given by $\mathscr{P}_{x 22_{1}}=-(1 / 2) \mathscr{S}^{-1}$ which in turn implies (Bamieh $\&$ Dahleh 2001)†

$$
f_{x}\left(k_{z}\right):=\operatorname{trace}\left(\mathscr{P}_{x 22_{1}}\left(k_{z}\right)\right)=\frac{2 k_{z} \operatorname{coth}\left(2 k_{z}\right)-1}{4 k_{z}^{2}} .
$$

On the other hand, application of Lemma 2 of Bamieh \& Dahleh (2001) to (E 15b) and $(\mathrm{E} 15 \mathrm{c})$, respectively yields

$$
\begin{aligned}
& \operatorname{trace}\left(\mathscr{P}_{y 11}\left(k_{z}\right)\right)=-\frac{1}{2} \operatorname{trace}\left(\mathscr{L}^{-1}\left(-k_{z}^{2} \Delta^{-1}\right)\right)=\frac{1}{2} k_{z}^{2} \operatorname{trace}\left(\left(\Delta^{2}\right)^{-1}\right), \\
& \operatorname{trace}\left(\mathscr{P}_{z 11}\left(k_{z}\right)\right)=-\frac{1}{2} \operatorname{trace}\left(\mathscr{L}^{-1} \Delta^{-1} \partial_{y y}\right)=-\frac{1}{2} \operatorname{trace}\left(\left(\Delta^{2}\right)^{-1} \partial_{y y}\right) .
\end{aligned}
$$

Therefore, the remaining problem amounts to determination of traces of operators $\left(\Delta^{2}\right)^{-1}$ and $\left(\Delta^{2}\right)^{-1} \partial_{y y}$ whose definitions are given by

$$
\begin{aligned}
& \left(\Delta^{2}\right)^{-1}: \quad f_{1} \longmapsto g_{1} \Leftrightarrow f_{1}=g_{1}^{(4)}-2 k_{z}^{2} g_{1}^{\prime \prime}+k_{z}^{4} g_{1}, \quad g_{1}( \pm 1)=g_{1}^{\prime}( \pm 1)=0, \\
& \left(\Delta^{2}\right)^{-1} \partial_{y y}: \quad f_{2} \longmapsto g_{2} \Leftrightarrow f_{2}^{\prime \prime}=g_{2}^{(4)}-2 k_{z}^{2} g_{2}^{\prime \prime}+k_{z}^{4} g_{2}, \quad g_{2}( \pm 1)=g_{2}^{\prime}( \pm 1)=0 .
\end{aligned}
$$

For notational convenience, we have suppressed the dependence of $f_{i}$ and $g_{j}$ on $k_{z}$, e.g. $f_{i}(y):=f_{i}\left(y, k_{z}\right), i=1$, 2. Similarly, $f_{i}^{(r)}(y):=\mathrm{d}^{r} f_{i}\left(y, k_{z}\right) / \mathrm{d} y^{r}$.

These two operators can be represented by their state-space realizations as follows

$$
\begin{aligned}
\left(\Delta^{2}\right)^{-1}:\left\{\begin{aligned}
x^{\prime}(y) & =\left[\begin{array}{cccc}
0 & 0 & 0 & -k_{z}^{4} \\
1 & 0 & 0 & 0 \\
0 & 1 & 0 & 2 k_{z}^{2} \\
0 & 0 & 1 & 0
\end{array}\right] x(y)+\left[\begin{array}{l}
1 \\
0 \\
0 \\
0
\end{array}\right] f_{1}(y), \\
g_{1}(y) & =\left[\begin{array}{llll}
0 & 0 & 0 & 1
\end{array}\right] x(y), \\
0 & =\left[\begin{array}{ll}
0_{2 \times 2} & \boldsymbol{I}_{2 \times 2} \\
0_{2 \times 2} & 0_{2 \times 2}
\end{array}\right] x(-1)+\left[\begin{array}{cc}
0_{2 \times 2} & 0_{2 \times 2} \\
0_{2 \times 2} & \boldsymbol{I}_{2 \times 2}
\end{array}\right] x(1), \quad y \in[-1,1],
\end{aligned}\right. \\
\left(\Delta^{2}\right)^{-1} \partial_{y y}:\left\{\begin{aligned}
z^{\prime}(y) & =\left[\begin{array}{llll}
0 & 0 & 0 & -k_{z}^{4} \\
1 & 0 & 0 & 0 \\
0 & 1 & 0 & 2 k_{z}^{2} \\
0 & 0 & 1 & 0
\end{array}\right] z(y)+\left[\begin{array}{l}
0 \\
0 \\
1 \\
0
\end{array}\right] f_{2}(y), \\
g_{2}(y) & =\left[\begin{array}{llll}
0 & 0 & 0 & 1
\end{array}\right] z(y), \\
0 & =\left[\begin{array}{lll}
0_{2 \times 2} & \boldsymbol{I}_{2 \times 2} \\
0_{2 \times 2} & 0_{2 \times 2}
\end{array}\right] z(-1)+\left[\begin{array}{ll}
0_{2 \times 2} & 0_{2 \times 2} \\
0_{2 \times 2} & \boldsymbol{I}_{2 \times 2}
\end{array}\right] z(1), \quad y \in[-1,1] .
\end{aligned}\right.
\end{aligned}
$$

$\dagger$ Expression (27) in Bamieh \& Dahleh (2001) should read $\left(2 k_{z} \operatorname{coth}\left(2 k_{z}\right)-1\right) /\left(4 k_{z}^{2}\right)$. 
The realizations of both operators are in form (E 13) and thus, formula (E 14) can be used to determine their traces. Since both realizations are parameterized by spanwise wavenumber, the traces of underlying operators are $k_{z}$-dependent as well. With the help of MATHEMATICA we obtain the following formulae for $\operatorname{trace}\left(\mathscr{P}_{y 11}\left(k_{z}\right)\right)$ and $\operatorname{trace}\left(\mathscr{P}_{z 11}\left(k_{z}\right)\right)$ :

$$
\begin{gathered}
f_{y}\left(k_{z}\right)=\frac{1}{2} k_{z}^{2} \operatorname{trace}\left(\left(\Delta^{2}\right)^{-1}\right)=-\frac{16 k_{z}^{4}+24 k_{z}^{2}+3 k_{z} \sinh \left(4 k_{z}\right)-9 \sinh ^{2}\left(2 k_{z}\right)}{24 k_{z}^{2}\left(4 k_{z}^{2}-\sinh ^{2}\left(2 k_{z}\right)\right)}, \\
f_{z}\left(k_{z}\right)=-\frac{1}{2} \operatorname{trace}\left(\left(\Delta^{2}\right)^{-1} \partial_{y y}\right)=\frac{16 k_{z}^{4}-3 k_{z} \sinh \left(4 k_{z}\right)+3 \sinh ^{2}\left(2 k_{z}\right)}{24 k_{z}^{2}\left(4 k_{z}^{2}-\sinh ^{2}\left(2 k_{z}\right)\right)} .
\end{gathered}
$$

It worth mentioning that the above determined traces do not depend on the form of channel flow. On the other hand, the traces of operators $\mathscr{P}_{y 22_{2}}\left(k_{z}\right)$ and $\mathscr{P}_{z 22_{2}}\left(k_{z}\right)$ (that is, $g_{y}\left(k_{z}\right)$ and $g_{z}\left(k_{z}\right)$ ) are not nominal velocity independent. Rather, they depend on the underlying channel flow through its dependence on $U^{\prime}$. The derivation of the analytical expressions for these traces in Couette flow is also given in this section.

E.2.2. Formulae for $f_{u}\left(k_{z}\right), f_{v}\left(k_{z}\right), f_{w}\left(k_{z}\right)$, and $g_{u}\left(k_{z}\right)$

We recall that $f_{u}\left(k_{z}\right), f_{v}\left(k_{z}\right)$ and $f_{w}\left(k_{z}\right)$ are determined by $\operatorname{trace}\left(\mathscr{R}_{u 22}\left(k_{z}\right)\right)$, trace $\left(\mathscr{R}_{v 11_{1}}\left(k_{z}\right)\right)$ and trace $\left(\mathscr{R}_{w 11_{1}}\left(k_{z}\right)\right)$, where operators $\mathscr{R}_{u 22}, \mathscr{R}_{v 11_{1}}$ and $\mathscr{R}_{w 11_{1}}$, respectively, satisfy

$$
\begin{aligned}
\mathscr{S} \mathscr{R}_{u 22}+\mathscr{R}_{u 22} \mathscr{S} & =-\boldsymbol{I}, \\
\mathscr{L} \mathscr{R}_{v 11_{1}}+\mathscr{R}_{v 11_{1}} \mathscr{L} & =-\left(-k_{z}^{2} \Delta^{-1}\right), \\
\mathscr{L} \mathscr{R}_{w 11_{1}}+\mathscr{R}_{w 11_{1}} \mathscr{L} & =-\Delta^{-1} \partial_{y y} .
\end{aligned}
$$

Comparison of (E 15) and (E 18) implies

$$
\begin{aligned}
& f_{u}\left(k_{z}\right):=\operatorname{trace}\left(\mathscr{R}_{u 22}\left(k_{z}\right)\right)=\operatorname{trace}\left(\mathscr{P}_{x 22_{1}}\left(k_{z}\right)\right)=: f_{x}\left(k_{z}\right), \\
& f_{v}\left(k_{z}\right):=\operatorname{trace}\left(\mathscr{R}_{v 11_{1}}\left(k_{z}\right)\right)=\operatorname{trace}\left(\mathscr{P}_{y 11}\left(k_{z}\right)\right)=: f_{y}\left(k_{z}\right), \\
& f_{w}\left(k_{z}\right):=\operatorname{trace}\left(\mathscr{R}_{w 11_{1}}\left(k_{z}\right)\right)=\operatorname{trace}\left(\mathscr{P}_{z 11}\left(k_{z}\right)\right)=: f_{z}\left(k_{z}\right),
\end{aligned}
$$

where the expressions for $f_{x}, f_{y}$ and $f_{z}$ are given by (E 16) and (E 17). On the other hand, $g_{u}\left(k_{z}\right):=\operatorname{trace}\left(\mathscr{R}_{u 11_{2}}\left(k_{z}\right)\right)$ is a function of a nominal velocity. In Couette flow, the formula for $g_{u}\left(k_{z}\right)$ can be obtained by comparing the expression for $\left[\left\|\mathscr{H}_{2}\right\|_{2}^{2}\right]\left(k_{z}\right)$ of $\S 4$ with its counterpart of Bamieh \& Dahleh (2001). By doing so, we obtain the following formula:

$$
\begin{aligned}
& g_{u}\left(k_{z}\right):=\operatorname{trace}\left(\mathscr{R}_{u 11_{2}}\left(k_{z}\right)\right) \\
& =\frac{1}{4} \sum_{n=1}^{\infty} \frac{k_{z}^{2}}{\gamma_{n}^{4}}\left\{\begin{array}{l}
1-\frac{n^{2} \pi^{2}}{\gamma_{n}}\left[\frac{2 k_{z}\left(\cosh \left(2 k_{z}\right)-1\right)}{\gamma_{n}\left(\sinh \left(2 k_{z}\right)-2 k_{z}\right)}+\frac{1}{4\left(\alpha_{n} \operatorname{coth}\left(\alpha_{n}\right)-k_{z} \operatorname{coth}\left(k_{z}\right)\right)}\right] \\
1-\frac{n^{2} \pi^{2}}{\gamma_{n}}\left[\frac{2 k_{z}\left(\cosh \left(2 k_{z}\right)+1\right)}{\gamma_{n}\left(\sinh \left(2 k_{z}\right)+2 k_{z}\right)}+\frac{1}{4\left(\alpha_{n} \tanh \left(\alpha_{n}\right)-k_{z} \tanh \left(k_{z}\right)\right)}\right]
\end{array} n\right. \text { odd, }
\end{aligned}
$$

where

$$
\gamma_{n}:=-\left(\frac{n^{2} \pi^{2}}{4}+k_{z}^{2}\right), \quad \alpha_{n}:=\sqrt{2 k_{z}^{2}+\frac{n^{2} \pi^{2}}{4}}, \quad n \geqslant 1 .
$$

Our current efforts are directed towards development of the formula for $g_{u}\left(k_{z}\right)$ in Poiseuille flow. 
Structured external excitations: determination of nominal velocity independent traces

In this subsection, we consider streamwise constant LNS system (5.1) with 'structured' external excitations, and derive the explicit analytical expressions for functions $f_{x}, f_{y}$ and $f_{z}$ in Corollary 2. This is done for 'pre-modulation' in the wall-normal direction defined by (5.2). In this case, the nominal velocity independent operators (whose traces we want to determine) can be represented by a well-posed TPBVSR

$$
\mathscr{F}:\left\{\begin{aligned}
x^{\prime}(y) & =A x(y)+\mathrm{e}^{-2 a(y+1)} B f(y), \\
g(y) & =C x(y), \\
0 & =L_{1} x(-1)+L_{2} x(1), \quad y \in[-1,1],
\end{aligned}\right.
$$

where $A, B, C, L_{1}$ and $L_{2}$ denote $y$-independent matrices of appropriate dimensions. Thus, the traces of these operators can be obtained using the trace formula of Jovanović \& Bamieh (2005)

$$
\operatorname{trace}(\mathscr{F})=\frac{1-\mathrm{e}^{-4 a}}{4 a} \operatorname{tr}(C B)-\operatorname{tr}\left(\left(L_{1}+L_{2} \mathrm{e}^{2 A}\right)^{-1} L_{2}\left[\begin{array}{ll}
0 & I
\end{array}\right] \exp \left\{2\left[\begin{array}{cc}
A-2 a \boldsymbol{I} & 0 \\
B C & A
\end{array}\right]\right\}\left[\begin{array}{l}
\boldsymbol{I} \\
0
\end{array}\right]\right)
$$

This formula is employed to determine explicit analytical expressions for functions $f_{s}\left(k_{z}, a\right)$, for every $s=x, y, z$, in Corollary 2 .

E.2.3. Formulae for $f_{x}\left(k_{z}, a\right), f_{y}\left(k_{z}, a\right)$ and $f_{z}\left(k_{z}, a\right)$

We recall that $f_{x}\left(k_{z}, a\right), f_{y}\left(k_{z}, a\right)$ and $f_{z}\left(k_{z}, a\right)$ are determined by $\operatorname{trace}\left(\mathscr{P}_{x 22_{1}}\left(k_{z}, a\right)\right)$, $\operatorname{trace}\left(\mathscr{P}_{y 11}\left(k_{z}, a\right)\right)$ and $\operatorname{trace}\left(\mathscr{P}_{z 11}\left(k_{z}, a\right)\right)$, where operators $\mathscr{P}_{x 22_{1}}, \mathscr{P}_{y 11}$ and $\mathscr{P}_{z 11}$ respectively satisfy

$$
\begin{aligned}
\mathscr{S} \mathscr{P}_{x 22_{1}}+\mathscr{P}_{x 221} \mathscr{S} & =-\kappa^{2}, \\
\mathscr{L} \mathscr{P}_{y 11}+\mathscr{P}_{y 11} \mathscr{L} & =-\left(-k_{z}^{2} \Delta^{-1} \kappa^{2}\right), \\
\mathscr{L} \mathscr{P}_{z 11}+\mathscr{P}_{z 11} \mathscr{L} & =-\Delta^{-1}\left(2 \kappa \kappa^{\prime} \partial_{y}+\kappa^{2} \partial_{y y}\right) .
\end{aligned}
$$

Application of Lemma 2 of Bamieh \& Dahleh (2001) to (E 21a), (E 21b) and (E 21c) in combination with (5.2) yields

$$
\begin{gathered}
\operatorname{trace}\left(\mathscr{P}_{x 22_{1}}\left(k_{z}, a\right)\right)=-\frac{1}{2} \operatorname{trace}\left(\mathscr{S}^{-1} \kappa^{2}\right)=-\frac{1}{2} a^{2}(1+\operatorname{coth}(a))^{2} \operatorname{trace}\left(\Delta^{-1} \mathrm{e}^{-2 a(y+1)}\right) \\
\operatorname{trace}\left(\mathscr{P}_{y 11}\left(k_{z}, a\right)\right)=-\frac{1}{2} \operatorname{trace}\left(\mathscr{L}^{-1}\left(-k_{z}^{2} \Delta^{-1} \kappa^{2}\right)\right) \\
=\frac{1}{2} k_{z}^{2} a^{2}(1+\operatorname{coth}(a))^{2} \operatorname{trace}\left(\left(\Delta^{2}\right)^{-1} \mathrm{e}^{-2 a(y+1)}\right) \\
\operatorname{trace}\left(\mathscr{P}_{z 11}\left(k_{z}, a\right)\right)=-\frac{1}{2} \operatorname{trace}\left(\mathscr{L}^{-1} \Delta^{-1}\left(2 \kappa \kappa^{\prime} \partial_{y}+\kappa^{2} \partial_{y y}\right)\right) \\
=-\frac{1}{2} a^{2}(1+\operatorname{coth}(a))^{2} \operatorname{trace}\left(\left(\Delta^{2}\right)^{-1} \mathrm{e}^{-2 a(y+1)}\left(\partial_{y y}-2 a \partial_{y}\right)\right)
\end{gathered}
$$

Therefore, the remaining problem amounts to determination of traces of operators

$$
\begin{gathered}
\Delta^{-1} \mathrm{e}^{-2 a(y+1)}: f_{1} \longmapsto g_{1} \Leftrightarrow \mathrm{e}^{-2 a(y+1)} f_{1}=g_{1}^{\prime \prime}-k_{z}^{2} g_{1}, \\
\left(\Delta^{2}\right)^{-1} \mathrm{e}^{-2 a(y+1)}: f_{2} \longmapsto g_{2} \Leftrightarrow \mathrm{e}^{-2 a(y+1)} f_{2}=g_{2}^{(4)}-2 k_{z}^{2} g_{2}^{\prime \prime}+k_{z}^{4} g_{2}, \\
\left(\Delta^{2}\right)^{-1} \mathrm{e}^{-2 a(y+1)}\left(\partial_{y y}-2 a \partial_{y}\right): f_{3} \longmapsto g_{3} \Leftrightarrow \mathrm{e}^{-2 a(y+1)}\left(f_{3}^{\prime \prime}-2 a f_{3}^{\prime}\right)=g_{3}^{(4)}-2 k_{z}^{2} g_{3}^{\prime \prime}+k_{z}^{4} g_{3},
\end{gathered}
$$

with boundary conditions: $g_{1}( \pm 1)=0, g_{2}( \pm 1)=g_{2}^{\prime}( \pm 1)=0$, and $g_{3}( \pm 1)=$ $g_{3}^{\prime}( \pm 1)=0$. 
Operators $\Delta^{-1} \mathrm{e}^{-2 a(y+1)}$ and $\left(\Delta^{2}\right)^{-1} \mathrm{e}^{-2 a(y+1)}$ can be represented by their state-space realizations as follows

$$
\begin{gathered}
\Delta^{-1} \mathrm{e}^{-2 a(y+1)}:\left\{\begin{aligned}
q^{\prime}(y) & =\left[\begin{array}{cc}
0 & k_{z}^{2} \\
1 & 0
\end{array}\right] q(y)+\mathrm{e}^{-2 a(y+1)}\left[\begin{array}{l}
1 \\
0
\end{array}\right] f_{1}(y), \\
g_{1}(y)=\left[\begin{array}{ll}
0 & 1
\end{array}\right] q(y), & \\
0 & =\left[\begin{array}{ll}
0 & 1 \\
0 & 0
\end{array}\right] q(-1)+\left[\begin{array}{ll}
0 & 0 \\
0 & 1
\end{array}\right] q(1), \quad y \in[-1,1],
\end{aligned}\right. \\
\left(\Delta^{2}\right)^{-1} \mathrm{e}^{-2 a(y+1)}:\left\{\begin{aligned}
x^{\prime}(y)=\left[\begin{array}{cccc}
0 & 2 k_{z}^{2} & 0 & -k_{z}^{4} \\
1 & 0 & 0 & 0 \\
0 & 1 & 0 & 0 \\
0 & 0 & 1 & 0
\end{array}\right] x(y)+\mathrm{e}^{-2 a(y+1)}\left[\begin{array}{l}
1 \\
0 \\
0 \\
0
\end{array}\right] f_{2}(y), \\
g_{2}(y)=\left[\begin{array}{llll}
0 & 0 & 0 & 1
\end{array}\right] x(y), \\
0=\left[\begin{array}{lll}
0_{2 \times 2} & \boldsymbol{I}_{2 \times 2} \\
0_{2 \times 2} & 0
\end{array}\right] x(-1)+\left[\begin{array}{cc}
0_{2 \times 2} & 0_{2 \times 2} \\
0_{2 \times 2} & \boldsymbol{I}_{2 \times 2}
\end{array}\right] x(1), \quad y \in[-1,1] .
\end{aligned}\right.
\end{gathered}
$$

On the other hand, a realization of operator $\left(\Delta^{2}\right)^{-1} \mathrm{e}^{-2 a(y+1)}\left(\partial_{y y}-2 a \partial_{y}\right)$ is given by

$$
\left\{\begin{aligned}
z^{\prime}(y) & =\left[\begin{array}{cccc}
0 & 2 k_{z}^{2} & 0 & -k_{z}^{4} \\
1 & 0 & 0 & 0 \\
0 & 1 & 0 & 0 \\
0 & 0 & 1 & 0
\end{array}\right] z(y)+\mathrm{e}^{-2 a(y+1)}\left[\begin{array}{c}
2 k_{z}^{2} \\
2 a \\
1 \\
0
\end{array}\right] f_{3}(y), \\
g_{3}(y) & =\left[\begin{array}{llll}
0 & 0 & 0 & 1
\end{array}\right] z(y), \\
0 & =\left[\begin{array}{ll}
0_{2 \times 2} & \boldsymbol{I}_{2 \times 2} \\
0_{2 \times 2} & 0_{2 \times 2}
\end{array}\right] z(-1)+\left[\begin{array}{cc}
0_{2 \times 2} & 0_{2 \times 2} \\
0_{2 \times 2} & \boldsymbol{I}_{2 \times 2}
\end{array}\right] z(1), \quad y \in[-1,1] .
\end{aligned}\right.
$$

Note that, for any given pair $\left(k_{z}, a\right)$, the realizations of all three operators are of the form (E 19). We note that since all realizations are parameterized by spanwise wavenumber $k_{z}$ and parameter $a$, the traces of underlying operators are going to depend on $k_{z}$ and $a$ as well.

Using (E 20), with the help of MATHEMATICA we obtain the following formulae for traces of operators $\mathscr{P}_{x 22_{1}}\left(k_{z}, a\right), \mathscr{P}_{y 11}\left(k_{z}, a\right)$ and $\mathscr{P}_{z 11}\left(k_{z}, a\right)$

$$
f_{x}\left(k_{z}, a\right)= \begin{cases}\frac{a\left\{-a-a \operatorname{coth}^{2}(a)+2 k_{z} \operatorname{coth}\left(2 k_{z}\right) \operatorname{coth}(a)\right\}}{4\left(k_{z}^{2}-a^{2}\right)} & k_{z} \neq a \\ \frac{1}{8}\left\{1+\operatorname{coth}^{2}(a)-a \operatorname{csch}^{3}(a) \operatorname{sech}(a)\right\} & k_{z}=a\end{cases}
$$

$$
\begin{aligned}
& f_{y}\left(k_{z}, a\right) \\
& \quad= \begin{cases}\frac{\mathrm{e}^{-2 a} k_{z}^{2}(1+\operatorname{coth}(a))^{2}}{8 a\left(a^{2}-k_{z}^{2}\right)^{3}\left(1+8 k_{z}^{2}-\cosh \left(4 k_{z}\right)\right)} h_{y}\left(k_{z}, a\right) & k_{z} \neq a, \\
\frac{\operatorname{csch}^{2}(a)\left\{\left(3-288 a^{2}\right) \cosh (2 a)-3 \cosh (6 a)+8 a\left(21+32 a^{2}\right) \sinh (2 a)\right\}}{384\left(1+8 a^{2}-\cosh (4 a)\right)} & k_{z}=a,\end{cases}
\end{aligned}
$$




$$
\begin{aligned}
& f_{z}\left(k_{z}, a\right) \\
& \quad= \begin{cases}\frac{\mathrm{e}^{-4 a}(1+\operatorname{coth}(a))^{2}}{8 a\left(a^{2}-k_{z}^{2}\right)^{3}\left(1+8 k_{z}^{2}-\cosh \left(4 k_{z}\right)\right)} h_{z}\left(k_{z}, a\right) & k_{z} \neq a, \\
\frac{\operatorname{csch}^{2}(a)\left\{3\left(5+32 a^{2}\right) \cosh (2 a)-15 \cosh (6 a)+8 a\left(9+32 a^{2}\right) \sinh (2 a)\right\}}{384\left(1+8 a^{2}-\cosh (4 a)\right)} & k_{z}=a,\end{cases}
\end{aligned}
$$

where

$$
\begin{aligned}
h_{y}\left(k_{z}, a\right):= & a \cosh (2 a)\left\{a^{4}+\left(a k_{z}\right)^{2}\left(3+8 a^{2}\right)-16\left(a k_{z}^{2}\right)^{2}+8 k_{z}^{6}-a^{2}\left(a^{2}+3 k_{z}^{2}\right) \cosh \left(4 k_{z}\right)\right\} \\
& +k_{z} \sinh (2 a)\left\{-4\left(3 a^{4} k_{z}-4 a^{2} k_{z}^{3}+k_{z}^{5}\right)+a^{2}\left(3 a^{2}+k_{z}^{2}\right) \sinh \left(4 k_{z}\right)\right\}, \\
h_{z}\left(k_{z}, a\right):= & a \cosh (2 a)\left\{-8 k_{z}^{8}+2 a^{6}\left(1+8 k_{z}^{2}\right)+a^{4}\left(k_{z}^{2}-40 k_{z}^{4}\right)\right. \\
& \left.-a^{2}\left(2 a^{4}+\left(a k_{z}\right)^{2}+k_{z}^{4}\right) \cosh \left(4 k_{z}\right)+a^{2}\left(k_{z}^{4}+32 k_{z}^{6}\right)\right\}+k_{z} \sinh (2 a) \\
& \times\left\{4 k_{z}\left(-4 a^{6}+7 a^{4} k_{z}^{2}-4 a^{2} k_{z}^{4}+k_{z}^{6}\right)+a^{2}\left(4 a^{4}-a^{2} k_{z}^{2}+k_{z}^{4}\right) \sinh \left(4 k_{z}\right)\right\} .
\end{aligned}
$$

Determination of nominal velocity dependent traces

Next, we determine traces of nominal-velocity-dependent operators $\mathscr{P}_{s 22_{2}}, s=y$, $z$, for both 'unstructured' and 'structured' external excitations in Couette flow. While we are not able to solve for operators $\mathscr{P}_{y 22_{2}}$ and $\mathscr{P}_{z 22_{2}}$ explicitly, we are able to express their traces in terms of easily computable series. This is accomplished at the expense of doing a spectral decomposition of operators $\mathscr{L}$ and $\mathscr{S}$ (see Appendix B for details).

\section{E.2.4. Determination of $\operatorname{trace}\left(\mathscr{P}_{s 22_{2}}\right)$}

We recall that $g_{s}$, for every $s=y, z$, is determined by $\operatorname{trace}\left(\mathscr{P}_{s 22_{2}}\right)$, where operator $\mathscr{P}_{s 22_{2}}$ satisfies

$$
\begin{aligned}
\mathscr{L} \mathscr{P}_{s 11}+\mathscr{P}_{s 11} \mathscr{L} & =-\mathscr{M}_{s 11}, \\
\mathscr{S} \mathscr{P}_{s 0}+\mathscr{P}_{s 0} \mathscr{L} & =-\mathscr{C}_{p} \mathscr{P}_{s 11}, \\
\mathscr{S} \mathscr{P}_{s 22_{2}}+\mathscr{P}_{s 22_{2}} \mathscr{S} & =-\left(\mathscr{C}_{p} \mathscr{P}_{s 0}^{*}+\mathscr{P}_{s 0} \mathscr{C}_{p}^{*}\right)
\end{aligned}
$$

Solutions to (E 24a) and (E 24b) are respectively given by

$$
\begin{aligned}
& \mathscr{P}_{s 11}=\int_{0}^{\infty} \mathrm{e}^{\mathscr{L} t} \mathscr{M}_{s 11} \mathrm{e}^{\mathscr{L} t} \mathrm{~d} t \\
& \mathscr{P}_{s 0}=\int_{0}^{\infty} \mathrm{e}^{\mathscr{S} t} \mathscr{C}_{p} \mathscr{P}_{s 11} \mathrm{e}^{\mathscr{L} t} \mathrm{~d} t,
\end{aligned}
$$

and $\operatorname{trace}\left(\mathscr{P}_{s 22_{2}}\right)$ can be determined as

$$
g_{s}:=\operatorname{trace}\left(\mathscr{P}_{s 22_{2}}\right)=-\frac{1}{2} \operatorname{trace}\left(\mathscr{S}^{-1}\left(\mathscr{C}_{p} \mathscr{P}_{s 0}^{*}+\mathscr{P}_{s 0} \mathscr{C}_{p}^{*}\right)\right)
$$

Combination of (E 25b) and (E 26) yields

$$
\begin{aligned}
g_{s}= & -\frac{1}{2} \operatorname{trace}\left(\mathscr{S}^{-1} \mathscr{C}_{p} \mathscr{P}_{s 11} \int_{0}^{\infty} \mathrm{e}^{\mathscr{L} t} \mathscr{C}_{p}^{*} \mathrm{e}^{\mathscr{S} t} \mathrm{~d} t\right) \\
& -\frac{1}{2} \operatorname{trace}\left(\mathscr{S}^{-1} \mathscr{C}_{p} \int_{0}^{\infty} \mathrm{e}^{\mathscr{L} t} \mathscr{P}_{s 11} \mathscr{C}_{p}^{*} \mathrm{e}^{\mathscr{S} t} \mathrm{~d} t\right)=: g_{s_{1}}+g_{s_{2}} .
\end{aligned}
$$


The inherent difficulty is that we cannot determine the above integrals explicitly. However, as we now show, the traces can still be determined by carrying out a spectral decomposition of operators $\mathscr{L}$ and $\mathscr{S}$.

In order to evaluate $g_{s_{1}}$ and $g_{s_{2}}$ we need the following Lemma.

Lemma 5. Let $\left\{\gamma_{n}\right\}_{n \in \mathbb{N}},\left\{\varphi_{n}\right\}_{n \in \mathbb{N}}$ be the eigenvalues and eigenfunctions of the operator $\mathscr{S}$, then for any trace class operators $\mathscr{F}$ and $\mathscr{G}$

$$
\operatorname{trace}\left(\mathscr{F} \int_{0}^{\infty} \mathrm{e}^{\mathscr{L} t} \mathscr{G} \mathrm{e}^{\mathscr{S} t} \mathrm{~d} t\right)=-\sum_{n \in \mathbb{N}}\left\langle\varphi_{n}, \mathscr{F}\left(\mathscr{L}+\gamma_{n} I\right)^{-1} \mathscr{G} \varphi_{n}\right\rangle .
$$

Proof. Let the spectral decompositions of $\mathscr{S}$ and $\mathscr{L}$ be

$$
\mathscr{S}=\sum_{n \in \mathbb{N}} \gamma_{n} E_{n}^{\mathscr{S}}, \quad \mathscr{L}=\sum_{m \in \mathbb{N}} \lambda_{m} E_{m}^{\mathscr{L}},
$$

where $E_{n}^{\mathscr{S}}$ and $E_{n}^{\mathscr{L}}$ are the spectral projections of $\mathscr{S}$ and $\mathscr{L}$, respectively, that is $E_{n}^{\mathscr{S}} f:=\left\langle\varphi_{n}, f\right\rangle \varphi_{n}$ and $E_{m}^{\mathscr{L}} f:=\left\langle\sigma_{m}, f\right\rangle_{o s} \sigma_{m}$. We can then compute

$$
\begin{aligned}
\int_{0}^{\infty} \mathrm{e}^{\mathscr{L} t} \mathscr{G} \mathrm{e}^{\mathscr{S} t} \mathrm{~d} t & =\int_{0}^{\infty}\left\{\sum_{m \in \mathbb{N}} \mathrm{e}^{\lambda_{m} t} E_{m}^{\mathscr{L}}\right\} \mathscr{G}\left\{\sum_{n \in \mathbb{N}} \mathrm{e}^{\gamma_{n} t} E_{n}^{\mathscr{S}}\right\} \mathrm{d} t \\
& =\sum_{n \in \mathbb{N}} \sum_{m \in \mathbb{N}}\left\{\int_{0}^{\infty} \mathrm{e}^{\left(\lambda_{m}+\gamma_{n}\right) t} \mathrm{~d} t\right\} E_{m}^{\mathscr{L}} \mathscr{G} E_{n}^{\mathscr{S}} \\
& =-\sum_{n \in \mathbb{N}} \sum_{m \in \mathbb{N}} \frac{1}{\left(\lambda_{m}+\gamma_{n}\right)} E_{m}^{\mathscr{L}} \mathscr{G} E_{n}^{\mathscr{S}} \\
& =-\sum_{n \in \mathbb{N}}\left(\mathscr{L}+\gamma_{n} I\right)^{-1} \mathscr{G} E_{n}^{\mathscr{S}},
\end{aligned}
$$

where in the last equation we made a choice to recombine the spectral decomposition of $\mathscr{L}$. Now, the trace of any operator $\mathscr{H}$ can be calculated using any orthonormal basis set $\left\{\varphi_{i}\right\}_{i \in \mathbb{N}}$ by $\operatorname{trace}(\mathscr{H})=\sum_{i \in \mathbb{N}}\left\langle\varphi_{i}, \mathscr{H} \varphi_{i}\right\rangle$. Therefore

$$
\begin{aligned}
\operatorname{trace}\left(\mathscr{F} \int_{0}^{\infty} \mathrm{e}^{\mathscr{L} t} \mathscr{G} \mathrm{e}^{\mathscr{S} t} \mathrm{~d} t\right) & =-\operatorname{trace}\left(\mathscr{F} \sum_{n \in \mathbb{N}}\left(\mathscr{L}+\gamma_{n} \boldsymbol{I}\right)^{-1} \mathscr{G} E_{n}^{\mathscr{S}}\right) \\
& =-\sum_{i \in \mathbb{N}}\left\langle\varphi_{i}, \mathscr{F} \sum_{n \in \mathbb{N}}\left(\mathscr{L}+\gamma_{n} I\right)^{-1} \mathscr{G} E_{n}^{\mathscr{S}} \varphi_{i}\right\rangle \\
& =-\sum_{n \in \mathbb{N}}\left\langle\varphi_{n}, \mathscr{F}\left(\mathscr{L}+\gamma_{n} I\right)^{-1} \mathscr{G} \varphi_{n}\right\rangle .
\end{aligned}
$$

Similarly, by performing a spectral decomposition of $\mathscr{L}$ we can express $\mathscr{P}_{s 11}$ as

$$
\mathscr{P}_{s 11}=-\sum_{k \in \mathbb{N}}\left(\mathscr{L}+\lambda_{k} I\right)^{-1} \mathscr{M}_{s 11} E_{k}^{\mathscr{L}} .
$$

We are now able to express $g_{s_{1}}$ and $g_{s_{2}}$ as

$$
\begin{aligned}
g_{s_{1}} & =-\frac{1}{2} \operatorname{trace}\left(\mathscr{S}^{-1} \mathscr{C}_{p} \mathscr{P}_{s 11} \int_{0}^{\infty} \mathrm{e}^{\mathscr{L}_{t}} \mathscr{C}_{p}^{*} \mathrm{e}^{\mathscr{S} t} \mathrm{~d} t\right) \\
& =-\frac{1}{2} \sum_{n \in \mathbb{N}} \sum_{k \in \mathbb{N}}\left\langle\varphi_{n}, \mathscr{S}^{-1} \mathscr{C}_{p}\left(\mathscr{L}+\lambda_{k} \boldsymbol{I}\right)^{-1} \mathscr{M}_{s 11} E_{k}^{\mathscr{L}}\left(\mathscr{L}+\gamma_{n} I\right)^{-1} \mathscr{C}_{p}^{*} \varphi_{n}\right\rangle
\end{aligned}
$$




$$
\begin{aligned}
& =-\frac{1}{2} \sum_{n \in \mathbb{N}} \sum_{k \in \mathbb{N}}\left\langle\left(\mathscr{L}+\lambda_{k} \boldsymbol{I}\right)^{-1} \mathscr{C}_{p}^{*} \mathscr{S}^{-1} \varphi_{n}, \mathscr{M}_{s 11} E_{k}^{\mathscr{L}}\left(\mathscr{L}+\gamma_{n} \boldsymbol{I}\right)^{-1} \mathscr{C}_{p}^{*} \varphi_{n}\right\rangle_{o s} \\
& =-\frac{1}{2} \sum_{n \in \mathbb{N}} \sum_{k \in \mathbb{N}}\left\langle\left(\mathscr{L}+\gamma_{n} \boldsymbol{I}\right)^{-1} \sigma_{k}, \mathscr{C}_{p}^{*} \varphi_{n}\right\rangle_{o s}\left\langle\left(\mathscr{L}+\lambda_{k} \boldsymbol{I}\right)^{-1} \mathscr{C}_{p}^{*} \mathscr{S}^{-1} \varphi_{n}, \mathscr{M}_{s 11} \sigma_{k}\right\rangle_{o s},
\end{aligned}
$$

and

$$
\begin{aligned}
g_{s_{2}} & =-\frac{1}{2} \operatorname{trace}\left(\mathscr{S}^{-1} \mathscr{C}_{p} \int_{0}^{\infty} \mathrm{e}^{\mathscr{L} t} \mathscr{P}_{s 11} \mathscr{C}_{p}^{*} \mathrm{e}^{\mathscr{S} t} \mathrm{~d} t\right) \\
& =-\frac{1}{2} \sum_{n \in \mathbb{N}} \sum_{k \in \mathbb{N}}\left\langle\varphi_{n}, \mathscr{S}^{-1} \mathscr{C}_{p}\left(\mathscr{L}+\gamma_{n} \boldsymbol{I}\right)^{-1}\left(\mathscr{L}+\lambda_{k} \boldsymbol{I}\right)^{-1} \mathscr{M}_{s 11} E_{k}^{\mathscr{L}} \mathscr{C}_{p}^{*} \varphi_{n}\right\rangle \\
& =-\frac{1}{2} \sum_{n \in \mathbb{N}} \sum_{k \in \mathbb{N}}\left\langle\left(\mathscr{L}+\lambda_{k} \boldsymbol{I}\right)^{-1}\left(\mathscr{L}+\gamma_{n} \boldsymbol{I}\right)^{-1} \mathscr{C}_{p}^{*} \mathscr{S}^{-1} \varphi_{n}, \mathscr{M}_{s 11} E_{k}^{\mathscr{L}} \mathscr{C}_{p}^{*} \varphi_{n}\right\rangle_{o s} \\
& =-\frac{1}{2} \sum_{n \in \mathbb{N}} \sum_{k \in \mathbb{N}}\left\langle\sigma_{k}, \mathscr{C}_{p}^{*} \varphi_{n}\right\rangle_{o s}\left\langle\left(\mathscr{L}+\lambda_{k} \boldsymbol{I}\right)^{-1}\left(\mathscr{L}+\gamma_{n} \boldsymbol{I}\right)^{-1} \mathscr{C}_{p}^{*} \mathscr{S}^{-1} \varphi_{n}, \mathscr{M}_{s 11} \sigma_{k}\right\rangle_{o s} .
\end{aligned}
$$

In particular, for Couette flow we have

$$
\mathscr{C}_{p}^{*}=-\mathrm{i} k_{z} \Delta^{-1} \Rightarrow\left\{\begin{aligned}
\mathscr{C}_{p}^{*} \varphi_{n} & =-\frac{\mathrm{i} k_{z}}{\gamma_{n}} \varphi_{n}, \\
\mathscr{C}_{p}^{*} \mathscr{S}^{-1} \varphi_{n} & =-\frac{\mathrm{i} k_{z}}{\gamma_{n}^{2}} \varphi_{n},
\end{aligned}\right.
$$

which in turn implies

$$
\begin{aligned}
g_{s_{1}} & =-\frac{1}{2} \sum_{n \in \mathbb{N}} \sum_{k \in \mathbb{N}} \frac{k_{z}^{2}}{\gamma_{n}^{3}}\left\langle\left(\mathscr{L}+\gamma_{n} \boldsymbol{I}\right)^{-1} \sigma_{k}, \varphi_{n}\right\rangle_{o s}\left\langle\left(\mathscr{L}+\lambda_{k} \boldsymbol{I}\right)^{-1} \varphi_{n}, \mathscr{M}_{s 11} \sigma_{k}\right\rangle_{o s} \\
& =-\frac{1}{2} \sum_{n \in \mathbb{N}} \sum_{k \in \mathbb{N}} \frac{k_{z}^{2}}{\gamma_{n}^{3}} \frac{1}{\left(\lambda_{k}+\gamma_{n}\right)}\left\langle\sigma_{k}, \varphi_{n}\right\rangle_{o s}\left\langle\left(\mathscr{L}+\lambda_{k} \boldsymbol{I}\right)^{-1} \varphi_{n}, \mathscr{M}_{s 11} \sigma_{k}\right\rangle_{o s} \\
& =-\frac{1}{2} \sum_{n \in \mathbb{N}} \sum_{k \in \mathbb{N}} \sum_{m \in \mathbb{N}} \frac{k_{z}^{2}}{\gamma_{n}^{3}} \frac{1}{\left(\lambda_{k}+\gamma_{n}\right)\left(\lambda_{m}+\lambda_{k}\right)}\left\langle\sigma_{k}, \varphi_{n}\right\rangle_{o s}\left\langle\sigma_{m}, \varphi_{n}\right\rangle_{o s}\left\langle\sigma_{m}, \mathscr{M}_{s 11} \sigma_{k}\right\rangle_{o s},
\end{aligned}
$$

where we arrived at the last equality by performing the spectral decomposition of operator $\left(\mathscr{L}+\lambda_{k} I\right)^{-1}$. Similarly,

$$
\begin{aligned}
g_{s_{2}} & =-\frac{1}{2} \sum_{n \in \mathbb{N}} \sum_{k \in \mathbb{N}} \frac{k_{z}^{2}}{\gamma_{n}^{3}}\left\langle\sigma_{k}, \varphi_{n}\right\rangle_{o s}\left\langle\left(\mathscr{L}+\lambda_{k} I\right)^{-1}\left(\mathscr{L}+\gamma_{n} I\right)^{-1} \varphi_{n}, \mathscr{M}_{s 11} \sigma_{k}\right\rangle_{o s} \\
& =-\frac{1}{2} \sum_{n \in \mathbb{N}} \sum_{k \in \mathbb{N}} \sum_{m \in \mathbb{N}} \frac{k_{z}^{2}}{\gamma_{n}^{3}} \frac{1}{\left(\lambda_{m}+\lambda_{k}\right)\left(\lambda_{m}+\gamma_{n}\right)}\left\langle\sigma_{k}, \varphi_{n}\right\rangle_{o s}\left\langle\sigma_{m}, \varphi_{n}\right\rangle_{o s}\left\langle\sigma_{m}, \mathscr{M}_{s 11} \sigma_{k}\right\rangle_{o s} .
\end{aligned}
$$

Hence,

$$
g_{s}=-\frac{1}{2} \sum_{n, k, m \in \mathbb{N}} \frac{k_{z}^{2}}{\gamma_{n}^{3}} \frac{\lambda_{k}+\lambda_{m}+2 \gamma_{n}}{\left(\lambda_{m}+\lambda_{k}\right)\left(\lambda_{k}+\gamma_{n}\right)\left(\lambda_{m}+\gamma_{n}\right)}\left\langle\sigma_{k}, \varphi_{n}\right\rangle_{o s}\left\langle\sigma_{m}, \varphi_{n}\right\rangle_{o s}\left\langle\sigma_{m}, \mathscr{M}_{s 11} \sigma_{k}\right\rangle_{o s}
$$




$$
=-\frac{1}{2} k_{z}^{2} \sum_{n, k, m \in \mathbb{N}}\left\{\begin{array}{l}
\frac{\lambda_{1 k} \lambda_{1 m}\left(\lambda_{1 k}+\lambda_{1 m}+2 \gamma_{n}\right) A_{k} A_{m}(n \pi)^{2} \cos \left(p_{1 k}\right) \cos \left(p_{1 m}\right)}{\gamma_{n}^{3}\left(\lambda_{1 m}+\lambda_{1 k}\right)\left(\lambda_{1 k}^{2}-\gamma_{n}^{2}\right)\left(\lambda_{1 m}^{2}-\gamma_{n}^{2}\right)}\left\langle\sigma_{1 m}, \mathscr{M}_{s 11} \sigma_{1 k}\right\rangle_{o s} \\
\frac{\lambda_{2 k} \lambda_{2 m}\left(\lambda_{2 k}+\lambda_{2 m}+2 \gamma_{n}\right) B_{k} B_{m}(n \pi)^{2} \sin \left(p_{2 k}\right) \sin \left(p_{2 m}\right)}{\gamma_{n}^{3}\left(\lambda_{2 m}+\lambda_{2 k}\right)\left(\lambda_{2 k}^{2}-\gamma_{n}^{2}\right)\left(\lambda_{2 m}^{2}-\gamma_{n}^{2}\right)}\left\langle\sigma_{2 m}, \mathscr{M}_{s 11} \sigma_{2 k}\right\rangle_{o s} \\
n-\text { even, }
\end{array}\right.
$$

where

$$
\begin{aligned}
& p_{1 k} \tan \left(p_{1 k}\right)=-k_{z} \tanh \left(k_{z}\right), \lambda_{1 k}:=-\left(p_{1 k}^{2}+k_{z}^{2}\right), A_{k}:=\left\{\left(-\lambda_{1 k}\right)\left(1+\frac{\sin \left(2 p_{1 k}\right)}{2 p_{1 k}}\right)\right\}^{-1 / 2}, \\
& p_{2 k} \cot \left(p_{2 k}\right)=k_{z} \operatorname{coth}\left(k_{z}\right), \lambda_{2 k}:=-\left(p_{2 k}^{2}+k_{z}^{2}\right), B_{k}:=\left\{\left(-\lambda_{2 k}\right)\left(1-\frac{\sin \left(2 p_{2 k}\right)}{2 p_{2 k}}\right)\right\}^{-1 / 2},
\end{aligned}
$$

and

$$
\begin{aligned}
\sigma_{1 k} & :=A_{k}\left\{\cos \left(p_{1 k} y\right)-\frac{\cos \left(p_{1 k}\right)}{\cosh \left(k_{z}\right)} \cosh \left(k_{z} y\right)\right\}, \\
\sigma_{2 k} & :=B_{k}\left\{\sin \left(p_{2 k} y\right)-\frac{\sin \left(p_{2 k}\right)}{\sinh \left(k_{z}\right)} \sinh \left(k_{z} y\right)\right\} .
\end{aligned}
$$

Therefore, the remaining task amounts to evaluation of inner products $\left\langle\sigma_{1 m}\right.$, $\left.\mathscr{M}_{s 11} \sigma_{1 k}\right\rangle_{o s}$ and $\left\langle\sigma_{2 m}, \mathscr{M}_{s 11} \sigma_{2 k}\right\rangle_{o s}$ for $s=y, z$ for both 'unstructured' and 'structured' disturbances.

E.2.5. Formulae for $g_{y}\left(k_{z}\right)$ and $g_{z}\left(k_{z}\right)$ in Couette flow

We recall that for 'unstructured' disturbances $\mathscr{M}_{y 11}=-k_{z}^{2} \Delta^{-1}$ and $\mathscr{M}_{z 11}=\Delta^{-1} \partial_{y y}$. Thus,

$$
\left\langle\sigma_{i m}, \mathscr{M}_{y 11} \sigma_{i k}\right\rangle_{o s}=k_{z}^{2}\left\langle\sigma_{i m}, \sigma_{i k}\right\rangle_{2}, \quad\left\langle\sigma_{i m}, \mathscr{M}_{z 11} \sigma_{i k}\right\rangle_{o s}=-\left\langle\sigma_{i m}, \sigma_{i k}^{\prime \prime}\right\rangle_{2}, \quad \forall i=1,2,
$$

where $\sigma_{i k}^{\prime \prime}(y):=\mathrm{d}^{2} \sigma_{i k}(y) / \mathrm{d} y^{2}$. We evaluate these inner products in MATHEMATICA to obtain

$$
\begin{aligned}
& \left\langle\sigma_{1 m}, \mathscr{M}_{y 11} \sigma_{1 k}\right\rangle_{o s}= \begin{cases}k_{z}^{2} A_{k} A_{m} \cos \left(p_{1 k}\right) \cos \left(p_{1 m}\right)\left(\frac{\tanh \left(k_{z}\right)}{k_{z}}+\operatorname{sech}^{2}\left(k_{z}\right)\right), & m \neq k, \\
k_{z}^{2}\left\{A_{k}^{2} \cos ^{2}\left(p_{1 k}\right)\left(\frac{\tanh \left(k_{z}\right)}{k_{z}}+\operatorname{sech}^{2}\left(k_{z}\right)\right)-\frac{1}{\lambda_{1 k}}\right\}, & m=k,\end{cases} \\
& \left\langle\sigma_{2 m}, \mathscr{M}_{y 11} \sigma_{2 k}\right\rangle_{o s}= \begin{cases}k_{z}^{2} B_{k} B_{m} \sin \left(p_{2 k}\right) \sin \left(p_{2 m}\right)\left(\frac{\operatorname{coth}\left(k_{z}\right)}{k_{z}}-\operatorname{csch}^{2}\left(k_{z}\right)\right), & m \neq k, \\
k_{z}^{2}\left\{B_{k}^{2} \sin ^{2}\left(p_{2 k}\right)\left(\frac{\operatorname{coth}\left(k_{z}\right)}{k_{z}}-\operatorname{csch}^{2}\left(k_{z}\right)\right)-\frac{1}{\lambda_{2 k}}\right\}, & m=k,\end{cases} \\
& \left\langle\sigma_{1 m}, \mathscr{M}_{z 11} \sigma_{1 k}\right\rangle_{o s}= \begin{cases}-k_{z}^{2} A_{k} A_{m} \cos \left(p_{1 k}\right) \cos \left(p_{1 m}\right)\left(\frac{\tanh \left(k_{z}\right)}{k_{z}}+\operatorname{sech}^{2}\left(k_{z}\right)\right), & m \neq k, \\
1-k_{z}^{2}\left\{A_{k}^{2} \cos ^{2}\left(p_{1 k}\right)\left(\frac{\tanh \left(k_{z}\right)}{k_{z}}+\operatorname{sech}^{2}\left(k_{z}\right)\right)-\frac{1}{\lambda_{1 k}}\right\}, & m=k,\end{cases} \\
& \left\langle\sigma_{2 m}, \mathscr{M}_{z 11} \sigma_{2 k}\right\rangle_{o s}=\left\{\begin{array}{cl}
-k_{z}^{2} B_{k} B_{m} \sin \left(p_{2 k}\right) \sin \left(p_{2 m}\right)\left(\frac{\operatorname{coth}\left(k_{z}\right)}{k_{z}}-\operatorname{csch}^{2}\left(k_{z}\right)\right), & m \neq k, \\
1-k_{z}^{2}\left\{B_{k}^{2} \sin ^{2}\left(p_{2 k}\right)\left(\frac{\operatorname{coth}\left(k_{z}\right)}{k_{z}}-\operatorname{csch}^{2}\left(k_{z}\right)\right)-\frac{1}{\lambda_{2 k}}\right\}, & m=k .
\end{array}\right.
\end{aligned}
$$


E.2.6. Formulae for $g_{y}\left(k_{z}, a\right)$ and $g_{z}\left(k_{z}, a\right)$ in Couette flow

We recall that for 'structured' disturbances with 'pre-modulation' in $y$ given by (5.2)

$$
\begin{aligned}
& \mathscr{M}_{y 11}=-k_{z}^{2} a^{2}(1+\operatorname{coth}(a))^{2} \Delta^{-1} \mathrm{e}^{-2 a(y+1)}, \\
& \mathscr{M}_{z 11}=a^{2}(1+\operatorname{coth}(a))^{2} \Delta^{-1}\left\{\mathrm{e}^{-2 a(y+1)}\left(\partial_{y y}-2 a \partial_{y}\right)\right\} .
\end{aligned}
$$

Thus,

$$
\begin{array}{ll}
\left\langle\sigma_{i m}, \mathscr{M}_{y 11} \sigma_{i k}\right\rangle_{o s}=k_{z}^{2} a^{2}(1+\operatorname{coth}(a))^{2}\left\langle\sigma_{i m}, \mathrm{e}^{-2 a(y+1)} \sigma_{i k}\right\rangle_{2}, & \forall i=1,2, \\
\left\langle\sigma_{i m}, \mathscr{M}_{z 11} \sigma_{i k}\right\rangle_{o s}=-a^{2}(1+\operatorname{coth}(a))^{2}\left\langle\sigma_{i m}, \mathrm{e}^{-2 a(y+1)}\left(\sigma_{i k}^{\prime \prime}-2 a \sigma_{i k}^{\prime}\right)\right\rangle_{2}, & \forall i=1,2,
\end{array}
$$

where $\sigma_{i k}^{\prime}(y):=\mathrm{d} \sigma_{i k}(y) / \mathrm{d} y$. The analytical expressions for these inner products can be readily determined in MATHEMATICA.

\section{REFERENCES}

BAmien, B. \& DAhleh, M. 2001 Energy amplification in channel flows with stochastic excitation. Phys. Fluids 13, 3258-3269.

Berger, T. W., Kim, J., Lee, C. \& Lim, J. 2000 Turbulent boundary layer control utilizing the Lorentz force. Phys. Fluids 12, 631-649.

Berlin, S., Wiegel, M. \& Henningson, D. S. 1999 Numerical and experimental investigations of oblique boundary layer transition. J. Fluid Mech. 393, 23-57.

Bewley, T. R. \& LiU, S. 1998 Optimal and robust control and estimation of linear paths to transition. J. Fluid Mech. 365, 305-349.

Boyd, J. P. 1989 Chebyshev and Fourier Spectral Methods. Springer.

Butler, K. M. \& Farrell, B. F. 1992 Three-dimensional optimal perturbations in viscous shear flow. Phys. Fluids A 4, 1637.

Chapman, S. J. 2002 Subcritical transition in channel flows. J. Fluid Mech. 451, 35-97.

Crawford, C. H. \& Karniadakis, G. E. 1997 Reynolds stress analysis of EMHD-controlled wall turbulence. Part I. Streamwise forcing. Phys. Fluids 9, 788-806.

Curtain, R. F. \& ZWART, H. J. 1995 An Introduction to Infinite-Dimensional Linear Systems Theory. Springer.

DolPh, C. L. \& LewIS, D. C. 1958 On the application of infinite systems of ordinary differential equations to perturbations of plane Poiseuille flow. Q. Appl. Maths 16, 97-110.

DU, Y. \& KARNiADAKIS, G. E. 2000 Suppressing wall turbulence by means of a transverse traveling wave. Science 288, 1230-1234.

Du, Y., Symeonidis, V. \& KarniadaKis, G. E. 2002 Drag reduction in wall-bounded turbulence via a transverse travelling wave. J. Fluid Mech. 457, 1-34.

Elofsson, P. A. \& Alfredsson, P. H. 1998 An experimental study of oblique transition in plane Poiseuille flow. J. Fluid Mech. 358, 177-202.

Farrell, B. F. \& IoAnnou, P. J. 1993a Optimal excitation of three-dimensional perturbations in viscous constant shear flow. Phys. Fluids A 5, 1390-1400.

Farrell, B. F. \& IoAnNoU, P. J. $1993 b$ Stochastic forcing of the linearized Navier-Stokes equations. Phys. Fluids A 5, 2600-2609.

Goldstein, M. E. \& Hultgren, L. S. 1989 Boundary-layer receptivity to long-wave free-stream disturbances. Annu. Rev. Fluid Mech. 21, 137-166.

Gustavsson, L. H. 1991 Energy growth of three-dimensional disturbances in plane Poiseuille flow. J. Fluid Mech. 98, 149.

Henoch, C. \& Stace, J. 1995 Experimental investigation of a salt water turbulent boundary layer modified by an applied streamwise magnetohydrodynamic body force. Phys. Fluids 7 , 1371-1383.

HiLl, D. C. 1995 Adjoint systems and their role in the receptivity problem for boundary layers. J. Fluid Mech. 292, 183-204.

Högberg, M., Bewley, T. R. \& Henningson, D. S. $2003 a$ Linear feedback control and estimation of transition in plane channel flow. J. Fluid Mech. 481, 149-175. 
Högberg, M., Bewley, T. R. \& Henningson, D. S. $2003 b$ Relaminarization of $R_{e \tau}=100$ turbulence using linear state-feedback control. Phys. Fluids 15, 3572-3575.

Jovanović, M. R. 2004 Modeling, analysis, and control of spatially distributed systems. PhD thesis, University of California, Santa Barbara, available at http://www.ece.umn.edu/users/mihailo/.

Jovanović, M. R. \& BAMieH, B. 2001 Modelling flow statistics using the linearized Navier-Stokes equations. In Proc. of the 40th IEEE Conf. on Decision and Control, pp. 4944-4949. Orlando, FL.

Jovanović, M. R. \& Bamieh, B. 2005 A formula for frequency responses of distributed systems with one spatial variable. Systems Control Lett. (to appear), available at http://www.ece. umn.edu/users/mihailo/.

KiM, J. 2003 Control of turbulent boundary layers. Phys. Fluids 15, 1093-1105.

Kim, J. \& Lim, J. 2000 A linear process in wall-bounded turbulent shear flows. Phys. Fluids 12, $1885-1888$.

Kim, J., Morn, P. \& Moser, R. 1987 Turbulence statistics in fully developed channel flow at low Reynolds number. J. Fluid Mech. 177, 133-166.

Landahl, M. T. 1975 Wave breakdown and turbulence. SIAM J. Appl. Maths 28, 735-756.

LANDAHL, M. T. 1980 A note on an algebraic instability of inviscid parallel shear flows. J. Fluid Mech. 98, 243-251.

Lee, K. H., Cortelezzi, L., Kim, J. \& Speyer, J. 2001 Application of reduced-order controller to turbulent flows for drag reduction. Phys. Fluids 13, 1321-1330.

LuChini, P. \& BotTaRo, A. 1998 Görtler vortices: a backward-in-time approach to the receptivity problem. J. Fluid Mech. 363, 1-23.

ORR, W. M. F. 1907 The stability or instability of the steady motions of a perfect liquid and of a viscous liquid. Part I: A perfect liquid. Part II: A viscous liquid. Proc. R. Irish Acad. A 27, 9-138.

Reddy, S. C. \& Henningson, D. S. 1993 Energy growth in viscous channel flows. J. Fluid Mech. 252, 209-238.

Reddy, S. C., Schmid, P. J., Baggett, J. S. \& Henningson, D. S. 1998 On stability of streamwise streaks and transition tresholds in plane channel flows. J. Fluid Mech. 365, 269-303.

REYNOLDS, W. C. \& Kassinos, S. C. 1995 One-point modeling of rapidly deformed homogeneous turbulence. Proc. Math. Physical Sci. 451 (1941), 87-104.

Schmid, P. J. \& Henningson, D. S. 1992 A new mechanism for rapid transition involving a pair of oblique waves. Phys. Fluids A 4, 1986-1989.

Schmid, P. J. \& Henningson, D. S. 2001 Stability and Transition in Shear Flows. Springer.

Sirovich, L., Ball, K. S. \& KeEFe, L. R. 1990 Plane waves and structures in turbulent channel flow. Phys. Fluids A 2, 2217-2226.

Trefethen, L. N., Trefethen, A. E., Reddy, S. C. \& Driscoll, T. A. 1993 Hydrodynamic stability without eigenvalues. Science 261, 578-584.

VanMarcke, E. 1983 Random Fields: Analysis and Synthesis. MIT Press.

Zhou, K., Doyle, J. C. \& Glover, K. 1996 Robust and Optimal Control. Prentice Hall. 